\title{
Chemical Functionalization and Characterization of Graphene-based Materials
}

Giovanni Bottari, ${ }^{\diamond, \S, \neq} \mathrm{M}^{\mathrm{a}}$ Ángeles Herranz, ${ }^{ \pm}$Leonie Wibmer, ${ }^{\dagger}$ Michel Volland, ${ }^{\dagger}$ Laura Rodríguez-Pérez, ${ }^{ \pm}$Dirk M. Guldi, ${ }^{\dagger, *}$ Andreas Hirsch, ${ }^{\mp,{ }^{*}}$ Nazario Martín, ${ }^{ \pm, \neq, *}$ Francis $\mathrm{D}^{\prime}$ Souza ${ }^{*, *}$ and Tomás Torres ${ }^{\diamond, \S, \neq, *}$

$\checkmark$ Department of Organic Chemistry, Universidad Autónoma de Madrid, Cantoblanco, 28049 Madrid, Spain

${ }^{\S}$ Institute for Advanced Research in Chemical Sciences, Universidad Autónoma de Madrid, Cantoblanco, 28049 Madrid, Spain

${ }^{\neq}$IMDEA-Nanociencia, Campus de Cantoblanco, 28049 Madrid, Spain

${ }^{\dagger}$ Department of Chemistry and Pharmacy, Interdisciplinary Center for Molecular Materials (ICMM), Friedrich-Alexander-Universität Erlangen-Nürnberg, Egerlandstr. 3, 91058 Erlangen, Germany

${ }^{\uparrow}$ Department of Chemistry and Pharmacy, University Erlangen-Nürnberg, Henkestrasse 42, 91054 Erlangen, Germany

${ }^{ \pm}$Departamento de Química Orgánica I, Facultad de Ciencias Químicas, Universidad Complutense de Madrid, 28040 Madrid, Spain

$\ddagger$ Department of Chemistry, University of North Texas, 1155 Union Circle, 305070, 62035017, Denton, USA

E-mail: $\quad$ dirk.guldi@fau.de; $\quad$ andreas.hirsch@fau.de; nazmar@ucm.es; francis.dsouza@unt.edu; tomas.torres@uam.es

\begin{abstract}
Graphene-based materials (GBMs), with graphene, their most known member, at the head, constitute a large family of materials which has aroused the interest of scientists working in different research fields such as chemistry, physics, or materials science, to mention a few, arguably as no other material before. In this review, we offer a general overview on the most relevant synthetic approaches for the covalent and non-covalent functionalization and
\end{abstract}


characterization of GBMs. Moreover, some representative examples of the incorporation to GBMs of electroactive units such as porphyrins, phthalocyanines, or ferrocene, among others, affording donor-acceptor (D-A) hybrids are presented. For these latter systems, the photophysical characterization of their ground- and excited-state features has also been included, paying particular attention to elucidate the fundamental dynamics of the energy transfer and charge separation processes of these hybrids. For some of the presented architectures, their application in solar energy conversion schemes and energy production has been also discussed.

\section{Introduction}

Graphene is an allotrope of carbon constituted of a single atomic layer of $\mathrm{sp}^{2}$-hybridized carbon atoms in the form of a two-dimensional (2-D) hexagonal lattice and which can be considered as the fundamental structural component of other carbon allotropes like graphite or carbon nanotubes. Since its discovery, ${ }^{1}$ several graphene-based materials (GBMs) have joined the "graphene" family nurturing a research field spanning through a variety of disciplines including chemistry, physics, and materials science and which interest, in the last decade, has increased at a high pace. ${ }^{2}$ GBMs can be defined according to three fundamental compositional (i.e., the atomic carbon/oxygen ratio) and morphological features (i.e., i) the number of graphene layers, which determines the thickness, the specific surface area, and the bending elasticity of the material, and ii) the average lateral dimension, which determines the material's size and degree of deformability, properties which are important, for example, for biological applications). ${ }^{3}$

The use of compositional and morphological descriptors is necessary when dealing with GBMs since this broad family of materials embraces very different chemical species such as single-layer and few-layer graphene (i.e., 2-10 layers), graphene oxide (GO, normally as a single layer), reduced graphene oxide (rGO; normally a single layer), graphene nanosheets, ultrafine graphite (i.e., more than 10 graphene sheets but below $100 \mathrm{~nm}$ in thickness), graphene ribbons, and graphene dots.

As characterization tools of GBMs, several techniques are generally used. Thus, regarding the number of graphene layers suitable analytical techniques are transmission electron microscopy (TEM), atomic force microscopy (AFM), scanning tunneling microscopy 
(STM), and Raman spectroscopy, among others. ${ }^{4,5}$ The two former techniques, as well as scanning electron microscopy (SEM), are also routinely used to obtain information regarding the GBMs' average lateral dimension. Finally, the atomic $\mathrm{C} / \mathrm{O}$ ratio is commonly determined by X-ray photoelectron spectroscopy (XPS), elemental analysis, and inductively coupled plasma mass spectrometry (ICP-MS).

This review will be devoted to some aspects of GBMs, namely, the characterization and chemical functionalization of these carbon materials. For some GBM-based donor-acceptor (D-A) hybrids, their photoinduced excited state features will also be discussed with the aim of rationalizing which effect the structural and electronic nature of GBMs have on the photoinduced charge/energy transfer dynamics, with a focus on energy production.

The review starts with the characterization of GBMs by STM and Raman spectroscopy. On one hand, STM allows gathering valuable information about the morphology and electronic structure of GBMs. On the other hand, Raman is a versatile technique used to spectroscopically characterize GBMs, as it assists in distinguishing, for example, the number of layers, defects or crystal disorder, edge structures, changes in the hybridization, and doping effects, etc. ${ }^{6}$ These aspects are of fundamental importance for the chemical functionalization of graphene for which both covalent and non-covalent approaches - both constituting the next part of the review - have been established.

The covalent functionalization of graphene represents a very challenging and, at the same time, important research field within the science and technology of the so-called "synthetic carbon allotropes". It requires the formation of covalent bonds onto the basal plane of the carbon lattice, an addition which changes the hybridization at the addend-carrying graphene carbon atoms from $\mathrm{sp}^{2}$ to $\mathrm{sp}^{3}$. This process can be formally considered as an introduction of defects and leads to the modification of the electronic (band structure), optical, and mechanical properties of the 2-D carbon allotrope. Moreover, the covalent functionalisation offers the opportunity to improve the solubility and processability of graphene, which would be otherwise difficult to master in terms of practical applications. Furthermore, the covalent functionalization allows for the combination of the unique properties of graphene with those of photo- and/or electroactive compounds. ${ }^{7,8} \mathrm{Up}$ to date, several strategies have been used for the covalent functionalization of GBMs, most of them employing synthetic protocols previously applied to the functionalization of fullerenes or carbon nanotubes. 
Synthetic strategies involving the addition to graphene of phenyl radicals, ${ }^{9}$ diazonium compounds, ${ }^{10-13}$ azomethyne ylids, ${ }^{14}$ fluorinated phenyl nitrenes, ${ }^{15}$ carbenes, ${ }^{16}$ or DielsAlder cycloaddition reactions ${ }^{17}$ have been explored allowing to incorporate a wide variety of electroactive units to the GBMs. While some of these works have been recently reviewed $^{18-21}$ - therefore they will be only briefly presented in the last part of this revision together with the physicochemical properties of graphene-based D-A hybrids - in the corresponding section we will focus on a much less explored covalent functionalization strategy for GBMs, namely their reductive covalent chemistry. This strategy, based on the formation of negatively charged graphitic species, offers some interesting advantages with respect to the more classical functionalization strategies such as a higher degree of functionalization of the GBMs. Concomitantly to the covalent functionalization of GBMs, the non-covalent functionalization of these materials has also been actively pursued. ${ }^{22}$ This latter strategy is particularly attractive since, in most of the cases, and opposite to the covalent functionalization, allows preserving the conjugated $\pi$-system of graphene. By virtue of its 2-D structure, GBMs represent an ideal platform for the preparation of tailored hybrid materials through non-covalent interactions with opto- and electronically-active organic components. For the non-covalent functionalization of GBMs, molecule-tographene intermolecular forces such as $\pi-\pi$, cation- $\pi$, hydrogen- $\pi$ and gas- $\pi$ have been explored; strategies that were effectively used earlier in the context of carbon nanotubes. ${ }^{23,24}$ In the corresponding section, we present some recent examples of the noncovalent modification of graphene through liquid phase exfoliation and solution-based processes. The ligands usually combined with graphene as binding partners are surfactants, polymers, or small aromatic molecules. This functionalization strategy has been recently reviewed in some specialized journals ${ }^{25-30}$ and here only a few representative examples are summarized. A section dealing with the non-covalent functionalization of epitaxial graphene growth on solid surfaces and the study of the resulting hybrids by ultra-high vacuum STM has also been included.

The last part of this review is devoted to the study of the photophysical properties of D-A systems based on GMBs. During the last decades, an enormous effort has been made towards the preparation of model compounds of natural photosynthetic systems with the ultimate goal of studying and understanding energy conversion processes. To this end, a 
wide range of covalent and non-covalent D-A systems have been designed, frequently using carbon nanostructures like fullerenes, carbon nanotubes and, more recently, graphene-type materials - particularly few-layer graphene, GO, and rGO - as the electron acceptors - or donors, although to a much lesser extend - species. This last section starts describing D-A systems based on monolayer and few-layer graphene, to then move towards hybrids integrating chemically modified graphene such as GO and rGO. In each subtopic, both covalent and non-covalent methodologies have been separately discussed, with a reference, for some of these systems, to the synthetic strategies used to prepare them. Moreover, for some of the presented D-A hybrids, their use in solar energy conversion schemes have been also included.

\section{Basics of scanning tunnelling microscopy (STM) and Raman spectroscopy of GBMs}

One of the first methods to optically characterize GBMs was scanning tunnelling microscopy (STM). Considering resolution down to the atomic level, information about morphology and electronic structure of GBMs were gathered. Topographical mapping provided insights into the coupling within the top layers. For example, in few layer graphene, there are different stacking modes. Firstly, AA-stacking, where the layers are directly stacked above each other with all carbon atoms on top of each other (Figure 1a). Secondly, the common AB- or Bernal stacking, where every second layer is shifted, so that their $\mathrm{A}$ atoms are above the underlying $\mathrm{B}$ atoms, and their $\mathrm{B}$ atoms above the centre of the underlying hexagons (Figure 1b). Thirdly, twisted variations, where the layers are rotated to each other (Fig. 1c,d). ${ }^{31,32}$ Each stacking mode is known to impact the electronic properties and, in turn, is monitored and differentiated by means of STM. In a perfect AB-stacking, the electron densities are seen as indefinite triangular lattices. For decoupled layers, honeycomb structures are found. A rotation of the layers results in so called Moiré patterns. The latter are a consequence of superposing and rotating identical periodic lattices (Figs $1 \mathrm{c}, \mathrm{d})$. 
a) AA stacking $\theta=0^{\circ}$

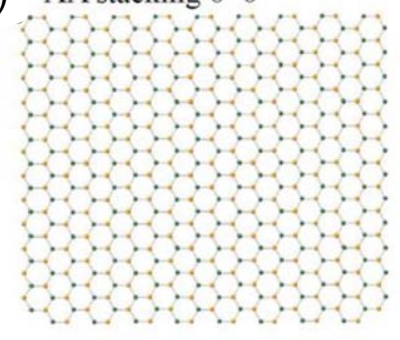

c)

$$
\theta=8^{\circ}
$$

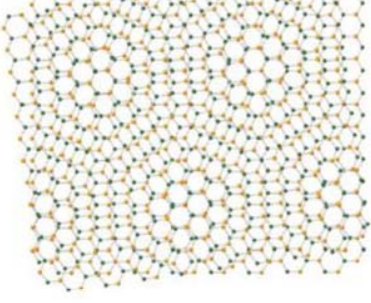

b) $\mathrm{AB}$ stacking $\theta=0^{\circ}$

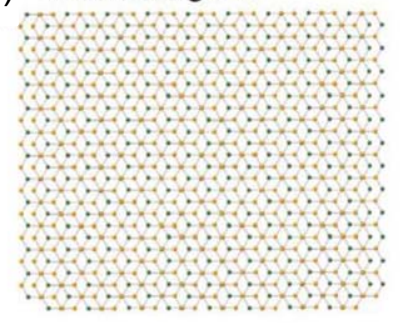

d)

$\theta=15^{\circ}$

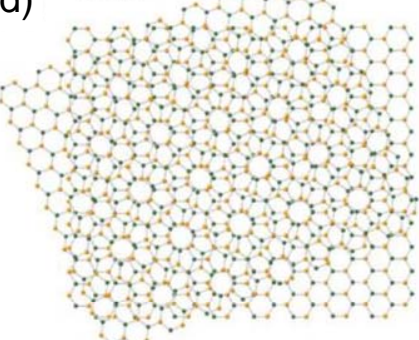

Fig. 1 Different possible stacking modes in graphite. a) AA-stacking, b) AB- or Bernal stacking, and emerging Moiré pattern when rotating two layers of graphene by c) $8^{\circ}$ or d) $15^{\circ}$. Reproduced with permission from ref. 31. Copyright 2017 Elsevier.

Due to challenges to investigate free standing graphene layers via STM, the method of choice was to grow perfect monolayers of graphene directly on a substrate and/or transfer them onto suitable substrates for STM investigations. One has to take caution as the underlying substrate plays an important role, especially in terms of influencing the obtained results.

The most common substrate, that is, insulating $\mathrm{SiO}_{2}$, has great drawbacks for the usage in STM. Potential fluctuations stemming from random impurities lead to the formation of so called electron hole puddles. As a matter of fact, false information about, for example, the lattice parameters and the couplings of the graphene layers are obtained. Moreover, the examination of the Dirac points via STS proved to be impossible due to too high deviations in energy at these points. ${ }^{33,34,35,36}$ Another possibility is based on the direct growth of graphene on metal substrates, such as $\mathrm{Ru}(111)^{37}$ or $\mathrm{Cu}(111) .{ }^{38}$ Here, STM corroborated that large-scale growth even along terrace steps is possible. Again, the electronic properties of the layers are influenced by the metal substrates leading to the Moiré substructures. In contrast to the aforementioned, using hBN substrates assists in reducing charge fluctuations and examining the low density regime and the Dirac point. Moiré patterns are still 
discernable, but due to slight variation in the coupling parameters between the A and B atoms in graphene and the boron and nitrogen atoms in $\mathrm{hBN}$ no sizable impact on the band gap of graphene evolves due to charge fluctuations. ${ }^{36,39}$ The best results are, however, obtained with graphite (Fig. 2). ${ }^{40}$ Here, sufficient decoupling from the substrate is reported. Pristine honeycomb structures lacking Moiré patterns and complete access to the electronic properties is provided.

a)

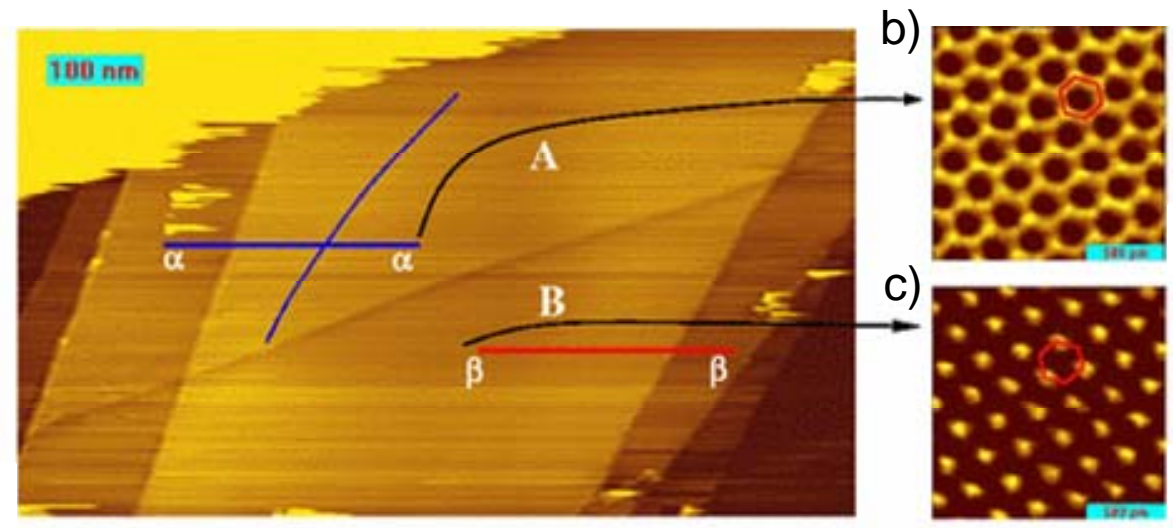

Fig. 2 a) STM image of graphene deposited onto graphite with two different areas: b) area A showing a honeycomb structure representing a decoupled single layer, and c) area B showing the triangular structure of Bernal stacked few-layer graphene. Reproduced with permission from ref. 40. Copyright 2009 American Physical Society.

Going to chemically modified graphene, for example, to graphene oxide, it becomes clear that, as STM relies largely on conductivity, GO features tremendous challenges to the measurements due to lattice distortions of the caused by functionalization: the tunnelling current decreases while the resistivity increases. Nevertheless, STM reveals direct insights into the structural changes on the atomic scale as this goes hand in hand with changes in the density of states.

As such, STM is used to follow the regeneration of the graphene like properties upon annealing highly reduced GO. It enables the complete restoration of a defective GO lattice structure by applying, for example, different annealing temperatures (Fig. 3). ${ }^{41}$ STM down to the atomic level yields direct insights in the underlying process, as remaining defects or vacancies are detected. A poorly conducting GO sheet is subject to an increase in 
conductivity and to an improvement of the image quality. Eventually, a characteristic hexagonal framework emerges. ${ }^{42,43,44}$

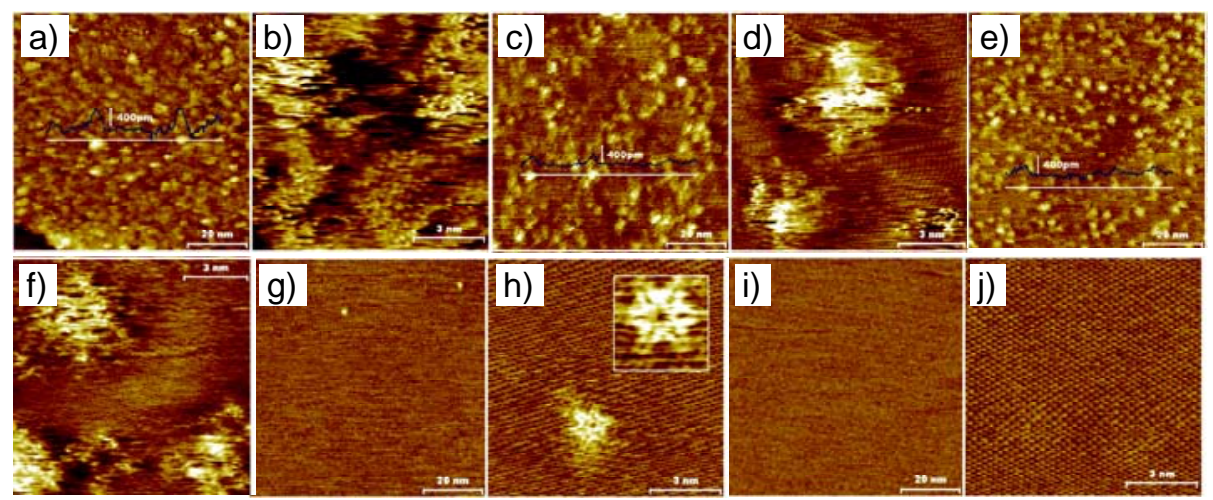

Fig. 3 Nanometer- and atomic-scale STM images for the highly reduced graphene oxide sheets prior to the thermal treatment $(a, b)$ after annealing at $1773 \mathrm{~K}(\mathrm{c}, \mathrm{d}), 1923 \mathrm{~K}(\mathrm{e}, \mathrm{f})$, $2073 \mathrm{~K}(\mathrm{~g}, \mathrm{~h})$ and $2223 \mathrm{~K}(\mathrm{i}, \mathrm{j})$. The inset to $(\mathrm{h})$ is a detailed $2 \times 2 \mathrm{~nm}^{2}$ image showing a different image of the same type of defect. Imaging conditions: $0.3 \mathrm{nA}$ (tunneling current) and $500 \mathrm{mV}$ (bias voltage) (a,c,e,g,i); 1-4 nA and 5-10 mV (b,d,f,j); $0.5 \mathrm{nA}$ and $80 \mathrm{mV}(\mathrm{h})$; $0.8 \mathrm{nA}$ and $50 \mathrm{mV}$ (inset). Reproduced with permission from ref. 41. Copyright 2014 American Chemical Society.

As STM provides details about the electronic density of the investigated specimen, it is a viable tool to detect changes resulting from doping. In terms of graphene, this tool is critical when probing heteroatom doped graphene sheets, namely doping with boron or nitrogen atoms by means of replacing carbon atoms in the hexagonal framework.

For $p$-doped graphene, boron is an outstanding element in terms of electron deficiency and Lewis acidity, yet being of comparable size with carbon. In this context, the a programmed synthesis of boron-doped graphene nanoribbons with a uniform distribution of Lewis acidic site has been recently reported with great potentials as a novel type of graphene transistors. ${ }^{45}$ Similarly, nitrogen atoms, which have a similar size compared to carbon, have been incorporated in the graphene nanostructures affording $n$-doped graphene sheets. With STM, it is possible to visualize these localized electron deficiency or excess and ultimately show the precise positions of the dopants in the graphene sheets as a function of electron density (Fig. 4). ${ }^{46,47}$ 


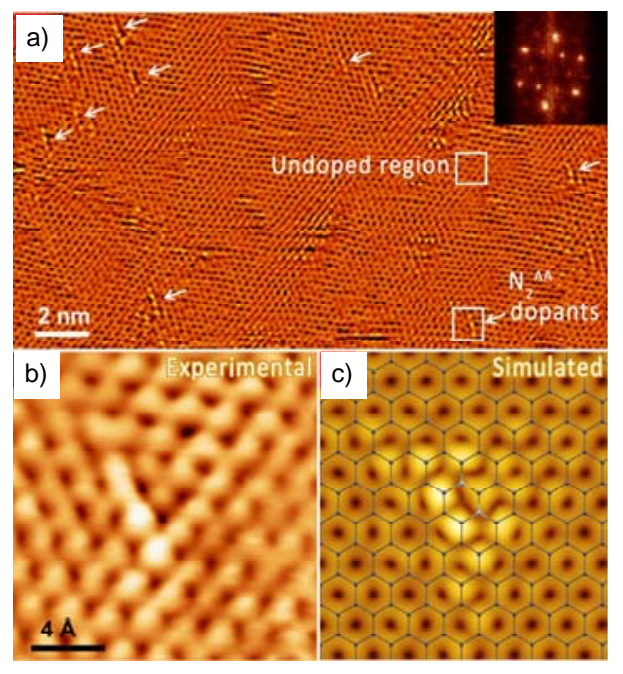

Fig. 4 (a) Large-area STM image of the NG illustrating the presence of numerous $\mathrm{N}$ dopants with similar peapod-like configuration (highlighted by white arrows), $\mathrm{V}_{\text {bias }}=-75$ $\mathrm{mV}, \mathrm{I}_{\text {set }}=100 \mathrm{pA}$. The upper and lower squares are used to indicate the undoped region and $\mathrm{N}_{2}{ }^{\mathrm{AA}}$ dopants. (Inset) FFT of topography presents reciprocal lattice (outer hexagon) and intervalley scattering (inner hexagon). The STM image shown here is obtained in flattening mode to remove the overall roughness of the substrate and enhance the atomic contrast of dopants. (b) Highly resolved STM image of $\mathrm{a} \mathrm{N}_{2}{ }^{\mathrm{AA}}$ dopant. (c) Ball-stick structural model of the $\mathrm{N}_{2}{ }^{\mathrm{AA}}$ dopant and simulated STM image obtained using first-principles calculations. The bias is $-1.0 \mathrm{eV}$. The carbon and nitrogen atoms are illustrated using gray and cyan balls, respectively. Reproduced with permission from ref. 47. Copyright 2012 Nature.

In such a way, a locally available description of the dopant position renders STM a key tool to characterize graphene based multifunctional materials for chemical sensing, nanoelectronics, photocatalysis, etc. It is, nevertheless, important to gather additional information, which is possible by Raman spectroscopy as it allows an even more precise investigation of graphene and graphenoid materials.

Turning to Raman, it allows to obtain important spectroscopic information regarding GBMs. For monolayer graphene, six phonon dispersion bands, three acoustic (A) and three optic $(\mathrm{O})$ branches, exist - Fig. $5{ }^{48,49}$ From the doubly degenerate phonon modes LO and iTO at the $\Gamma$ point the first order Raman $G$ band arises at $\sim 1580 \mathrm{~cm}^{-1}$. This $E_{2 \mathrm{~g}}$ symmetry vibration originates from $\mathrm{C}-\mathrm{C}$ stretch vibrations in a $\mathrm{sp}^{2}$ lattice. The G-band is subject to a Kohn anomaly, which renders it very sensitive to changes in the Fermi Level. At the K- 
point, second order processes give rise to the D- and the G'-band at $\sim 1350$ and $\sim 2700 \mathrm{~cm}^{-1}$, respectively. The D-band originates from one iTO phonon and a defect phonon, for example, at defects in the lattice or edges. Besides the D-band, another defect induced band, namely the D'-band, is located at $\sim 1620 \mathrm{~cm}^{-1}$ with its overtone, 2D'-band at $\sim 3200$ $\mathrm{cm}^{-1}$. The G'-band, although not a first order mode and located at approximately double the frequency of the D-band, is not due to defects, but evolves from two iTO phonons by a double resonance process. In the literature it is also often called "2D"-band. But, we prefer to refer to G'-band to avoid confusion and clarify its existence even in absence of defects, in contrast to the D- and D'-bands.
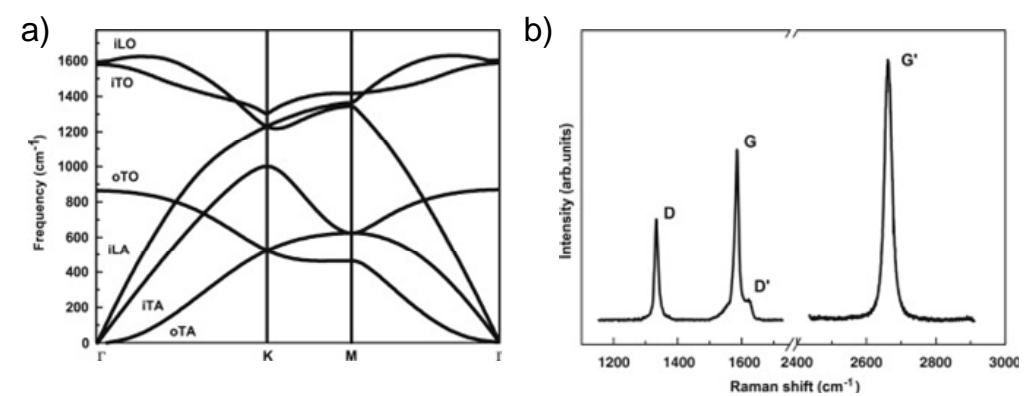

Fig. 5 a) Calculated phonon dispersion of graphene. b) Typical Raman spectrum of a graphene edge showing next to $G$ and $G$ '-bands also the defect induced D and D'-bands. Reproduced with permission from ref. 48. Copyright 2009 Elsevier.

This double resonance process then also enables the possibility to distinguish between graphene and graphite, and even the individual number of graphene layers - Fig. $6 .{ }^{48}$ For a single layer of graphene, the G'-band displays a single Lorentzian fit with a FWHM of $\sim 24$ $\mathrm{cm}^{-1}$ and a very high intensity originating from a triple resonance process only present in the perfect monolayer. Already in the bilayer, due to $\pi-\pi^{*}$ interactions between the two layers, more electron-phonon scattering processes are possible, which leads to a broadening of the G'-band. Now, four different processes contribute to the G'-band, where each Lorentzian fit displays a FWHM of $\sim 24 \mathrm{~cm}^{-1}$. Finally in HOPG, only two processes contribute to the double resonance and the G'-band is shifted to higher frequencies. 


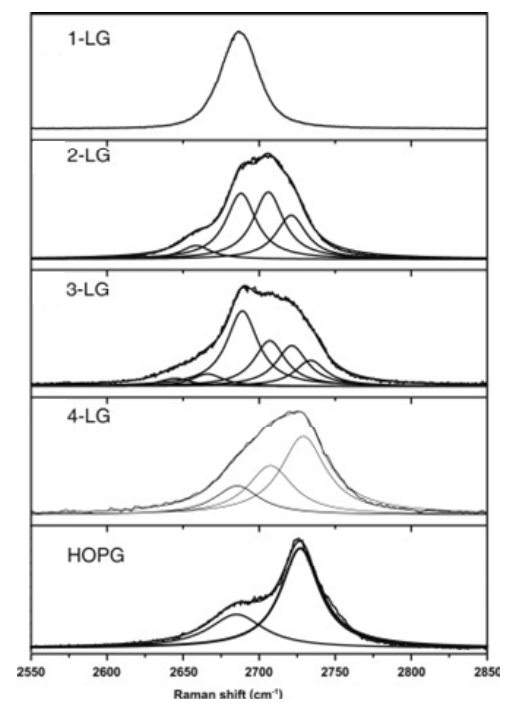

Fig. 6 Differences in the FWHM, the number of Lorentzian fits and the peak position of the G'-band depending on the number of layers. Reproduced with permission from ref. 48 . Copyright 2009 Elsevier.

Up to 4 layers roughly can be distinguished via the shape of the G'-band, before it appears like that of graphite. For all these cases, the layers stack with a regular AB Bernal stacking. ${ }^{50}$ In case of turbostratic graphite, where the layers restack in random order, the $\pi$ $\pi^{*}$ interactions between the layers is absent and, therefore, the G'-band displays again only a single Lorentzian fit. With that, turbostratic graphite shows a similar G'-band as a monolayer, but at upshifted frequencies and with a broader FWHM. Whereas the FWHM of a monolayer is $\sim 24 \mathrm{~cm}^{-1}$, that of turbostratic graphite is $\sim 45-60 \mathrm{~cm}^{-1}$. Next to turbostratic graphite, also folded flakes can occur, so called misoriented layers. For example, overlapping monolayers in random bilayer configurations also show only a single G'-peak, but at upshifted frequencies.

Shifts in the G'-band, and also the D-band, have to be considered with caution, as they are subject to a linear dispersive behaviour as the exciting laser power is varied - Fig. $7 .^{51,52}$ On the other hand, shifts might be significant when looking at doped graphene, because of the Kohn anomaly, for example, in non-covalent hybrids, GO, hydrogenated graphene, fluorinated fluorographene, or hydroxylated graphene. Most of the shifts relate to the defects when compared to pristine graphene, like changes in the $\mathrm{sp}^{2}$ hybridization, lattice distortions, and doping induced changes in the Fermi level. As the amount of defects is 
increased, the following trends are observed: Firstly, the D-band first increases in intensity, then decreases, broadens and upshifts in frequency. Secondly, the D'-band rises next to the G-band and the two merge into one broad band. Thirdly, the G'-band first decreases and broadens, then vanishes completely, and a D+D'-band arises. Depending on the nature of the defects, the aforementioned trends differ. Casiraghi et al. showed that by means of evaluating the $\mathrm{I}_{\mathrm{D}} / \mathrm{I}_{\mathrm{D}}$, and $\mathrm{I}_{\mathrm{D}} / \mathrm{I}_{\mathrm{G}}$ ratios information about the nature of the defects, that is, $\mathrm{sp}^{3}$ or vacancies, respectively, are obtained. ${ }^{53}$ Furthermore, the dependence of $\mathrm{I}_{\mathrm{D}} / \mathrm{I}_{\mathrm{D}}$, on the excitation wavelength differs with different defects.

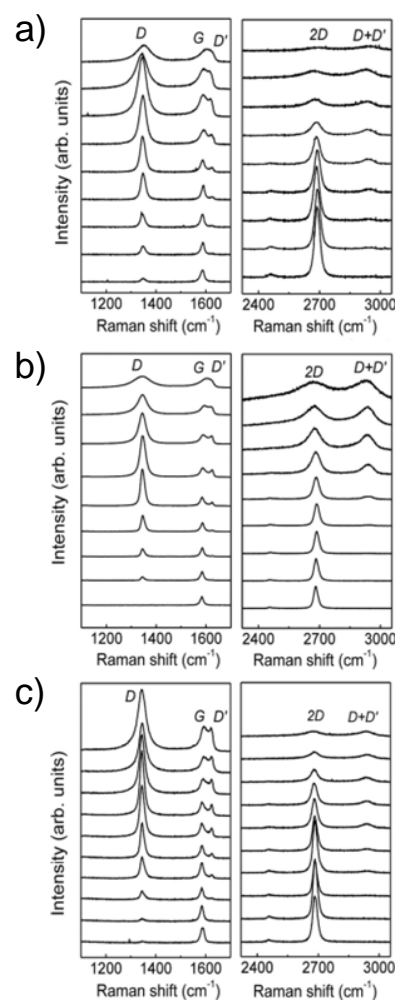

Fig. 7 Defect induced changes of the Raman spectra of a) fluorinated, b) oxidized, and c) anodic bonded graphene with increasing defect density (from bottom to top). Reproduced with permission from ref. 53. Copyright 2013 American Physical Society.

Specific type of defects are linked to edge effects. In particular, contributions stemming from edge effects in the Raman spectra of nanoribbons are of great importance to distinguish between zigzag and armchair conformations. The D-band of a zigzag-like structure is, for example, lower in intensity than that of an armchair conformation - 
perfectly grown zigzag structures lack any D-band. The D-band contribution of the zigzag derives only from potential disorder. ${ }^{54}$

Furthermore, Jorio et al. confirmed that the G-band exhibits an intensity dependence on the light polarization relative to the ribbon axis - Fig. $8 .^{55}$ Thus, the G-band presents another way to distinguish zigzag from armchair conformation, as well as, edges from interior regions of the ribbon.

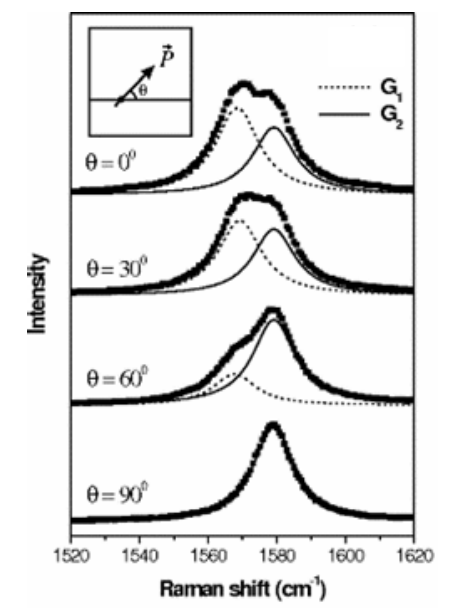

Fig. 8 Polarization angle depending Raman spectra with respect to carbon ribbon direction. Reproduced with permission from ref. 55. Copyright 2004 American Physical Society.

In the case of non-covalent immobilization onto the basal plane, planar aromatic molecules are the dopants of choice, since the planarity and their high electron density are useful to facilitate the interactions of both entities. Another approach is to deposit metals onto the basal plane: cobalt, for example, as electron and nickel as hole dopant. ${ }^{56}$

Li et al. showed that by monitoring the G- and G'-bands of the sample and comparing it to a reference, besides an overall broadening of the two modes, $p$-doping ( $n$-doping) results in an upshift (downshift) of the G-band as well as an upshift (upshift) of the G' band (Fig. 9). ${ }^{57}$ 


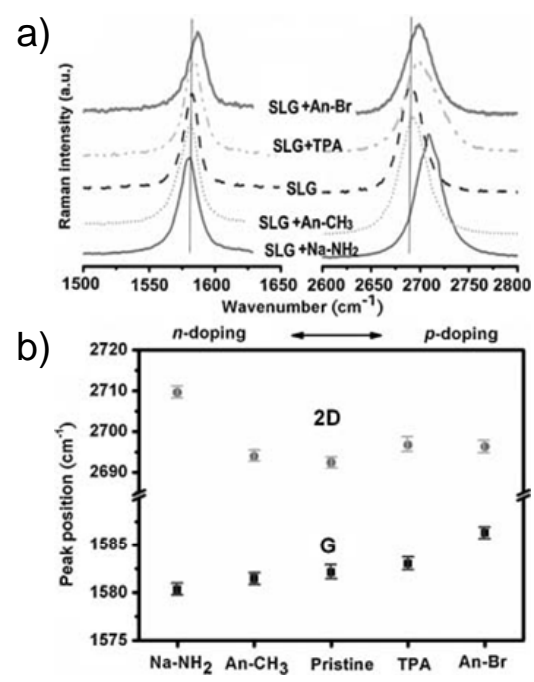

Fig. 9 a) Raman spectra with G (left) and G' (right) for doped single layer graphene with various aromatic molecules. b) Raman shifts for $G$ and G' (2D) band related to the doping effect. An-Br (9,10-dibromoanthracene), TPA (tetrasodium 1,3,6,8-pyrenetetrasulfonic acid), $\mathrm{An}-\mathrm{CH}_{3}$ (9,10-dimethylanthracene) and $\mathrm{Na}-\mathrm{NH}_{2}$ (1,5-naphthalene diamine). Reproduced with permission from ref. 57. Copyright 2009 Wiley-VCH.

In contrast to molecular doping, graphene can also be doped electrochemically by applying an external potential and, thus, injecting either holes or electrons. Dresselhaus et al. probed the Raman spectra of chemical vapour deposited (CVD) graphene in a range from -1.5 to $+1.5 \mathrm{~V}$ in $0.1 \mathrm{~V}$ steps and monitored changes in the position of the G-, as well as, in the G'band. The overall results were that, whereas, the G-band was upshifted in both cases, the G'-band upshifted in the case of hole doping (p-doping) and down for electron injection ( $n$ doping). These results are in sound agreement with the results obtained for non-covalent doping with planar aromatic molecules by Dong et al. described above. ${ }^{58-61}$

Another approach to alter the electronic structure of graphene is the selective covalent functionalization of graphene with possible dopants. Upon functionalization, the intensity of the D-band increases while, simultaneously, the G-band decreases in intensity due to the distortion of the $\mathrm{sp}^{2}$ hybridized framework. By varying the attached functional group it is possible to tailor the doping direction. Sandip et al. chose nitrophenyl as functional group for their experiments. Raman spectra, shown in Fig. 10 reveal $p$-doping of graphene due to 
nitrophenyl. Thus, band gap tailoring of graphene is possible with non-covalent as well as covalent approaches. ${ }^{62}$
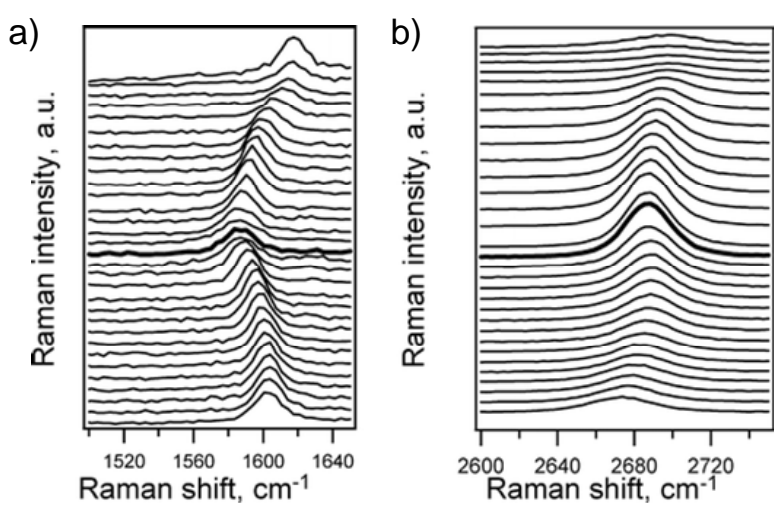

Fig. 10 Spectroelectrochemical Raman data upon excitation with $532 \mathrm{~nm}$ for the a) $\mathrm{G}$ and b) $\mathrm{G}^{\prime}$ band of graphene monitored for an applied potential range from -1.5 to $+1.5 \mathrm{~V}$ (bottom to top). The bold spectra correspond to $0 \mathrm{~V}$ while each additional spectra equals a potential change of $0.1 \mathrm{~V}$. Reproduced with permission from ref. 58. Copyright 2010 American Chemical Society.

$\mathrm{GO}$ and $\mathrm{rGO}$ are the most investigated graphene derivatives so far. It becomes clear, when speaking of $\mathrm{GO} / \mathrm{rGO}$ one has to take caution of the changes in the graphene lattice due to occurring defects. As discussed above, the arising of a D-band gives evidence for the introduced vacancies, as well as, the functional groups like oxygen. All of these distort the former perfect $\mathrm{sp}^{2}$ hybridized planar framework, yielding a broadening of all bands - Fig. 11. ${ }^{63}$ Hence, Ferrari et al. correlated the FWHM of the aforementioned graphene related Raman modes to the defect density. ${ }^{64,65}$ 


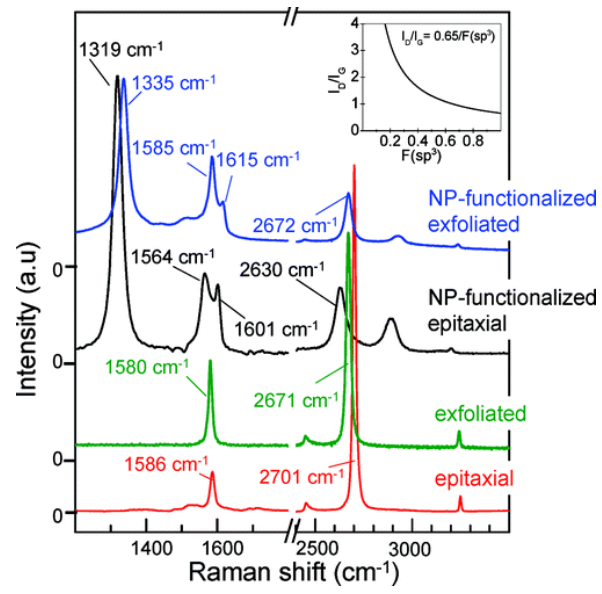

Fig. 11 Raman spectra of exfoliated and epitaxial graphene before and after covalent functionalization with nitrophenyl. Reproduced with permission from ref. 62. Copyright 2010 American Chemical Society.

In 2012, Hirsch et al. based their assumptions of the defect densities on the intensity ratios of $\mathrm{I}_{\mathrm{D}} / \mathrm{I}_{\mathrm{G}} .{ }^{66}$ They performed statistical Raman microscopy and evaluated data of a distinct area of the sample, recording thousands of locally resolved spectra - Fig. 12. With that information at hand, they proposed a simple tool to directly link the integrity of the carbon lattice to the $\mathrm{I}_{\mathrm{D}} / \mathrm{I}_{\mathrm{G}}$ ratio, in accordance with aforementioned results of Ferrari et al. ${ }^{65}$

a)

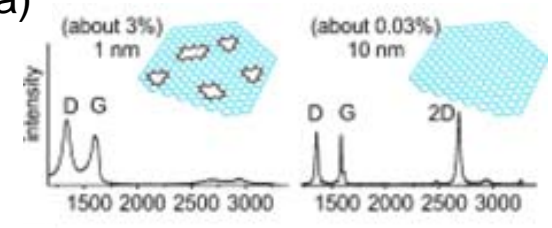

b)

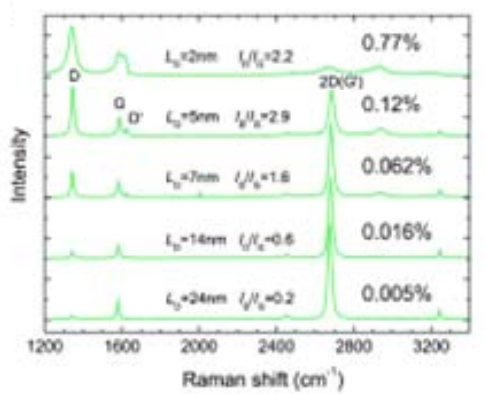

Fig. 12 a) Raman spectra of GO with different defect densities. Reproduced with permission from ref. 66. Copyright 2014 Wiley-VCH. b) Selected Raman spectra with 
defined defect densities. Reproduced with permission from ref. 65. Copyright 2011 American Chemical Society.

In 2016, Minett et al. showed, however, that the methodology to link the $\mathrm{I}_{\mathrm{D}} / \mathrm{I}_{\mathrm{G}}$ intensity ratio to the inter defect distances is not applicable to GO - Fig. $13 .{ }^{67}$ As a matter of fact, the overall broadening leads to a merging of the G- and D'-band into a single signal, and, thus, distort the obtained ratio. They called the superimposed feature $\mathrm{G}_{\mathrm{a}}$-band. Their concept involved the overtone of the D'-band, namely 2D' at $3200 \mathrm{~cm}^{-1}$, rather than the D'-band, as the 2D'-band of rGO occurs at higher frequencies compared to GO. Their measure to quantify the defect ratio and the quality of the resulting rGO, is the difference in the positions of the 2D'-band compared to the G-band. ${ }^{68-71}$

a)

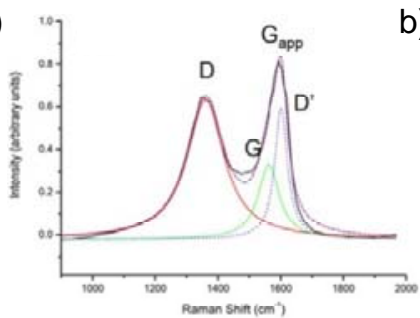

c)

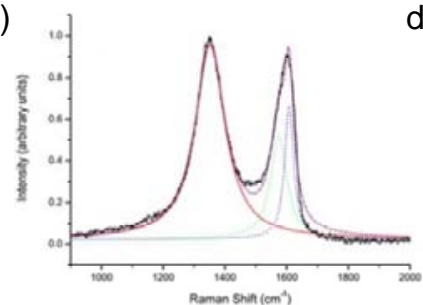

b)

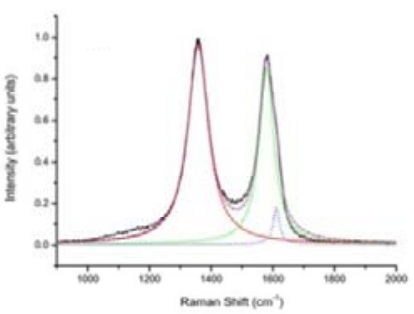

d)

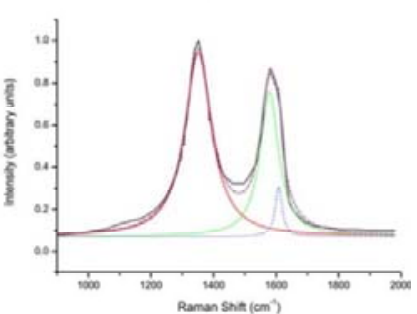

Fig. 13 Raman spectra of a) GO before thermal reduction at $1000^{\circ} \mathrm{C}$, b) GO after thermal reduction, c) GO before reduction with hydrazine, and d) GO after reduction with hydrazine. Reproduced with permission from ref. 67. Copyright 2016 Nature Publishing Group.

Novoselov et al. synthesized and characterized the first hydrogenated graphene, so called graphane. ${ }^{72}$ Upon hydrogenation by plasma treating, $\mathrm{sp}^{3}$ defects are introduced that give rise to D- and D'-bands, in accordance with the examined defect structures. ${ }^{73}$ They monitored the degree of the hydrogenation by comparing a free standing sample and a sample with an underlying substrate - Fig. 14. The D-band was twice as intense for the freestanding sample, proofing the possible hydrogenation from two sides instead of one. 
Annealing experiments showed, furthermore, an upshift in the G- and G'-bands, from which $p$-doping was concluded. Hirsch et al. performed the synthesis of graphane in the liquid phase via a Birch reduction, but annealing of the samples was necessary to obtain qualitative Raman spectra. ${ }^{74}$ To this end, the typical Raman bands for hydrogenated graphene are recovered, revealing an $\mathrm{I}_{\mathrm{D}} / \mathrm{I}_{\mathrm{G}}$ ratio of approximately $1: 1$ and a broadened $\mathrm{G}^{\prime}-$ band.
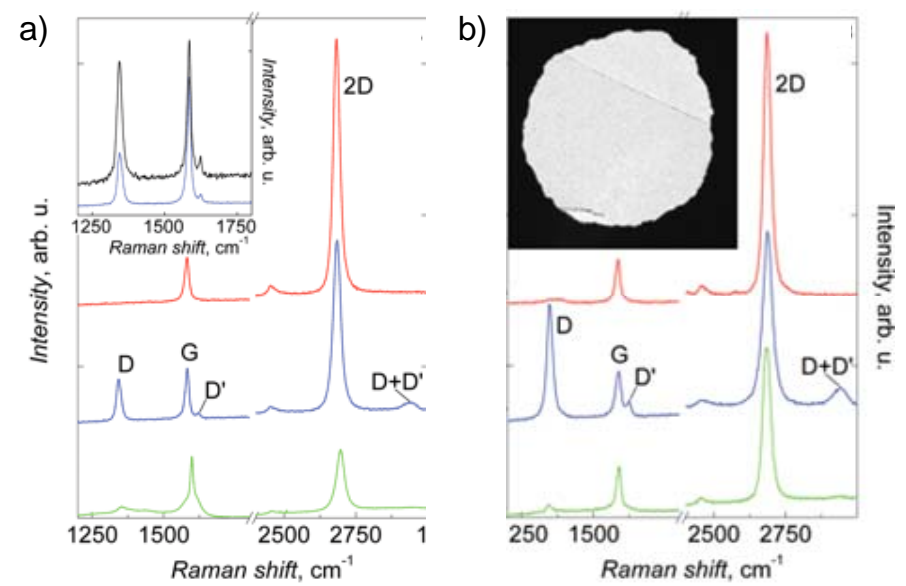

Fig. 14 Differences in the Raman spectra of a) graphene on a $\mathrm{SiO}_{2}$ substrate, and b) free standing graphene membrane with its TEM image in the right inset, before (green) and after hydrogenation (blue), and after annealing (red). Reproduced with permission from ref. 72. Copyright 2009 American Association for the Advancement of Science.

Another well examined graphene is fluorographene. Geim et al. showed that during fluorination, for an initially formed partial fluorinated graphene the spectra resemble those found for GO, with increasing D-band and decreasing G'-band - Fig. 15. ${ }^{75}$ After further fluorination, the underlying transformation of graphene to a wide band gap material leads to a lack of Raman activity upon using visible light. Then, Zhu et al. applied UV excitation to monitor C-F vibrations at $\sim 1270$ and $\sim 1345 \mathrm{~cm}^{-1}$. ${ }^{76}$ 


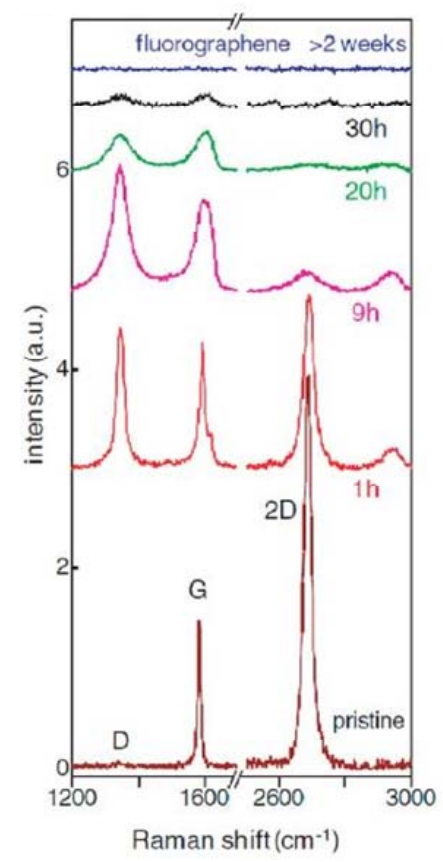

Fig. 15 Changes in the Raman spectra as a function of exposure to atomic fluorine. Reproduced with permission from ref. 75. Copyright 2010 Wiley-VCH.

\section{Reductive covalent functionalization of graphene}

The covalent chemistry of graphene which is basically addition of suitable reactive building blocks to the conjugated $\pi$-system of the carbon lattice is still in its infancy. ${ }^{77-82}$ Typical examples are the addition of phenyl radicals, ${ }^{9}$ diazonium compounds, ${ }^{10-13}$ azomethyne ylids, ${ }^{14}$ fluorinated phenyl nitrenes, ${ }^{15}$ carbenes, ${ }^{16}$ as well as Diels-Alder cycloaddition reactions. ${ }^{17}$ A general problem of all of these reactions is the rather low degree of functionalization. Moreover, it is still very challenging to unambiguously prove the success of covalent addition chemistry, since classical characterization tools of synthetic organic chemistry such as NMR spectroscopy or mass spectrometry cannot be applied. The most important aspects of the introduction of new and reliable characterisation tools for covalent graphene functionalization will be pointed out below.

The comparatively low degree of functionalisation of most covalent additions to neutral graphene is due to the fact that graphene is a rather inert system. Because of its planar structure and the lack of any carbon atom pyramidalization it is much less reactive with respect to initial additions than fullerenes or carbon nanotubes. In order to overcome the 
problem of low reactivity, Hirsch and co-workers have recently developed a new concept for graphene functionalisation which allows for the generation of a large variety of covalent adducts exhibiting comparatively high degrees of addition, which at the same time can easily be modified by varying the reaction conditions. ${ }^{83}$ The key point is the use of negatively charged graphites or graphenides, the so called graphite intercalation compounds (GICs). In GICs alkaline metals such as potassium are placed in between in the sheets of the graphite starting material. They have been known for a very long time and have been studied in quite some detail both what the physical and chemical properties is concerned. The advantage of using negatively charged graphene sheets (graphenides) instead of normal graphite/graphene is the fact that i) the negative charging introduces Columbic repulsion of the graphene sheets allowing for improved dispersion in solvents, and ii) chemical activation because of the high degree of reduction. The electron charging of the graphene sheets can in principle have two chemical consequences namely, i) electron transfer to suitable reagents leading to radicals which can subsequently undergo addition reactions with the graphene sheet, and ii) nucleophilic additions of the graphene carbon atoms to suitable electrophiles.

Next to these two possible reaction pathways, one has also to consider a very important topological aspect associated with covalent graphene functionalisation. This is related to the way how the graphene sheets are presented in a chemical reaction. If the graphene sheets are homogeneously dispersed in a suitable medium, attacks from both sides of the graphene plane can in principle take place. This addition mode can lead to comparatively strain free addition geometries. If on the other hand the graphene is deposited on a surface, then attacks from only one side can take place. In this case the subsequent additions to neighbouring carbon atoms within the graphene sheet lead to rather bent and strained binding geometries. As a consequence lower degrees of additions are expected. Within this section, we will differentiate between these binding scenarios and compare wet chemical bulk functionalization with the monotopic addition to graphene sheets supported on a surface.

As a first example of the reductive covalent functionalisation of graphene, the treatment of potassium graphenides with aryl diazonium salts was investigated (Scheme 1). ${ }^{83}$ The in situ reduction was carried by the treatment with a sodium/potassium alloy $\left(\mathrm{NaK}_{3}\right)$ in 1,2- 
dimethoxyethane (DME) as an inert electride/alkalide stabilizing solvent. The subsequent addition of the diazonium salt dispersion to the reduced and exfoliated graphene sheets was accompanied by vigorous nitrogen evolution.

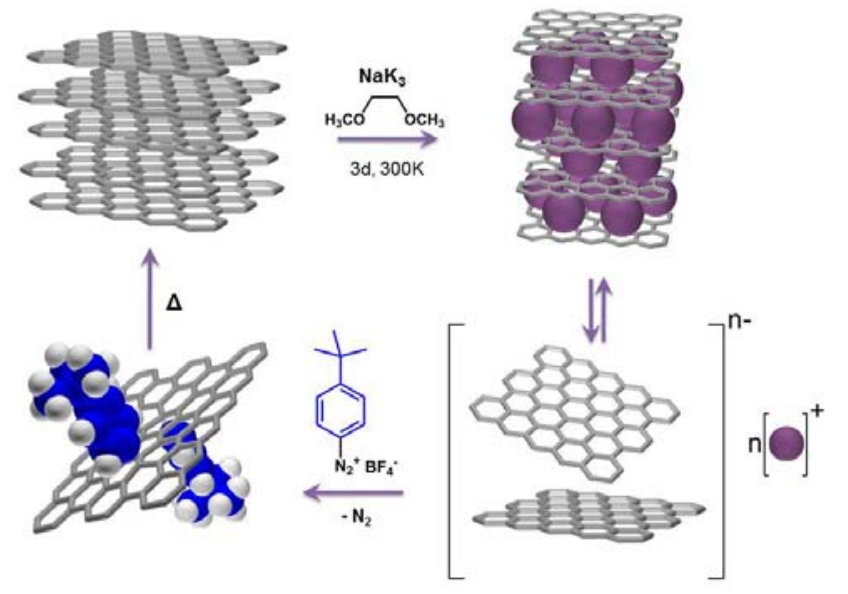

Scheme 1 Reductive covalent functionalization of GICs with phenyl diazonium tetrafluoroborate. Reproduced with permission from ref. 83. Copyright 2011 Nature Publishing Group.

The structural analysis of the reaction product by high resolution transition electron microscopy (HR-TEM) revealed the formation of domains of highly arylated regions located next to intact nanographene islands within the flake. Obviously, no homogeneous functionalization has taken place. Raman spectroscopy is among the most powerful methods to analyse covalently functionalised graphene products. The introduction of $\mathrm{sp}^{3}$ defects due to covalent addend binding becomes apparent by an increase of the D peak. At the same time the two 2D peak sharpens and becomes more symmetrical finally reaching a Lorentzian shape which is characteristic for a decoupled graphene monolayer. In order to get even more information about the outcome of the addition reactions statistical Raman spectroscopy (SRS) and statistical Raman microscopy (SRM) by analysing spatially resolved Raman mappings was introduced. ${ }^{83,84}$ Annealing of the reaction products at elevated temperatures (up to $1000{ }^{\circ} \mathrm{C}$ ) led to the complete regeneration of the hexagonal $\mathrm{sp}^{2}$ carbon framework and the restauration of graphite (Scheme 1). This clearly demonstrates that the covalent graphene functionalisation is a reversible process that can be used as a 
vehicle for the modification of graphene resulting in increased solubilisation which can be very helpful for materials processing. Since reduced graphite in form of GICs applied in this study is a very good reducing agent and since the reaction is accompanied by vigorous nitrogen evolution one can assume that the first reduction pathway (see above), namely the reduction of the aryl diazonium salts accompanied by cleavage of $\mathrm{N}_{2}$ and a subsequent attack of phenyl radicals to the graphene framework is the predominant reaction mechanism.

Next to diazonium salts also alkyl iodides have been used as reaction partners for the covalent functionalisation of GICs. ${ }^{85}$ In a systematic study three different graphite starting materials namely flake-graphite, powder graphite and spherical graphite have been employed. It was demonstrated that also the alkylation of graphite using alkyl iodides is possible. Interestingly, the degree of addition depends on the nature of the graphite starting material. The morphology and crystallinity of the graphite starting material as well as the flake size determines the degree of functionalisation, the exfoliation efficiency and the product homogeneity. The highest degree of functionalisation of $3.6 \%$ was determined for the alkylated graphene powder. This result can be interpreted by the fact, that graphene powder exhibits the lowest bulk density in comparison to graphene-flakes and spherical graphene. As a consequence, the in situ reduction and the resulting dispersion in organic media is most efficient in this type of graphite starting material.

In a study comparing the reactivity of carbon nanotubes with that of graphene, $\lambda$-iodanes were used as reaction partners for GICs. In order to tune the degree of functionalisation different ratios (i.e., 1:4, 1:8, 1:16 and 1:24) of potassium to carbon in the corresponding GIGs where applied. As in the case of the aryl diazonium compounds and also the alkyl iodides, the hypervalent iodine compounds easily form radicals and can therefore be used as suitable reagents in these functionalisation sequences. ${ }^{86}$ The corresponding functionalisation efficiency was determined by thermogravimetric analysis (TGA= measurements. A linear correlation between the degree of negative charging and the incremental mass loss promoted by thermal cleavage was observed. The coupling of TGA, mass spectrometry and gas chromatography analysis unambiguously allowed the assignment of the thermal cleavage products, the corresponding masses and elution properties of the cleaved addends. For the first time, it was possible to separate and to quantify the 
detached addends formerly covalently linked to the carbon lattice of graphene through thermogravimetric GS MS measurements.

In order to carry out reductive hydrogenation of graphene in the bulk a different approach has been established. For this purpose, a Birch-type reaction sequence to generate polyhydrogenated graphene has been applied (Scheme 2). ${ }^{87}$ The Birch reduction is widely used for hydrogenation of PAHs and carbon allotropes. ${ }^{88-90}$ In the first reaction step one equivalent of spherical graphite flakes was intercalated with a five-fold excess of lithium metal in liquid ammonia at $-78^{\circ} \mathrm{C}$. After the addition of 10 equivalents of deionized water, the ammonia was evaporated and the mixture was allowed to warm to room temperature.

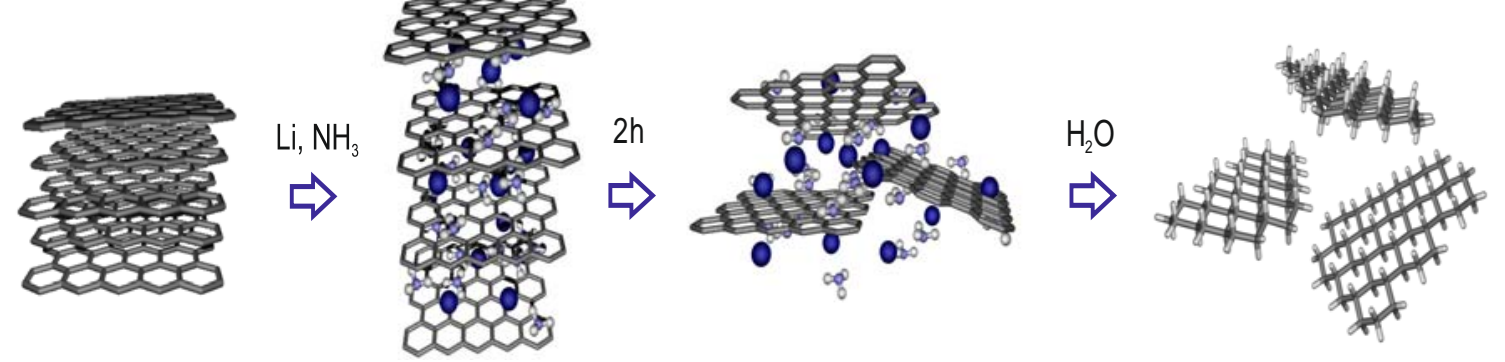

Scheme 2 Schematic representation of the Birch-type hydrogenation of graphene. Reproduced with permission from ref. 87. Copyright 2013 Wiley-VCH.

Interestingly, the alkalide solution retained its reducing activity after the addition of water which was indicated by the persistence of the blue colour of the solvated electrons. No significant $\mathrm{H}_{2}$ formation took place at this stage. It turned out that water which under these reaction conditions exists as frozen ice is the most suitable proton source. If alcohols where used instead the degree of hydrogenation where significantly lower and also $\mathrm{H}_{2}$ evolution was determined. Obviously, the reaction with ice on the surface leads to a very gentle supply of protons and an efficient suppression of hydrogen evolution. The corresponding reaction product appears as a golden/brown powder. This already indicates rather extensive covalent hydrogenation and extensive rupture of the $\pi$-conjugation. Systematic TGA studies clearly showed the loss of $\mathrm{H}_{2}$ after heating the sample to over $400{ }^{\circ} \mathrm{C}$. Interestingly, the typical Raman features of graphene/graphite are lost in the reaction product and only very brought and structure less features appear. However, the Raman spectrum of graphite can be restored if the sample is heated to over $700{ }^{\circ} \mathrm{C}$. The restauration of these Raman 
features was followed by subsequent heating steps of $100{ }^{\circ} \mathrm{C}$ and the simultaneous recording of the corresponding Raman spectra. These measurements revealed continuous regaining of the graphite features. For further unambiguous structural characterisation also the corresponding deuteration of graphene has been carried out by using deuterated ammonia and deuterated water as deuterium source. The clear proof for the successful deuteration was obtained from IR-measurements where the circular dichroism vibration at about $2135 \mathrm{~cm}^{-1}$ was observed clearly demonstrating the expected isotope shift. In contrast, the corresponding hydrogenated compounds showed the $\mathrm{CH}$ vibrations at $2852 \mathrm{~cm}^{-1}$. Interestingly, the polyhydrogenated graphene shows yellow fluorescence even in the solid state. This can be explained by the presence of a band gap and the change of the electronic properties generated by the high degree of functionalisation. From excitation of the polyhydrogenated graphene with a conventional portable UV-lamp $\left(\lambda_{\text {exc }}=366 \mathrm{~nm}\right)$ a bright yellow emission can be seen. Broad emission features spreading from 450 to $650 \mathrm{~nm}$ are observed at an excitation at $280 \mathrm{~nm}$. This broad spectrum indicates the presence of predominantly isolated and randomly $\mathrm{nm}$-sized graphene domains that all contribute independently to the fluorescence events. Obviously, the hydrogenation does not take place in a homogenous fashion but instead the formation of highly hydrogenated islands which are located next to intact nanographene regions takes place. Similar results have already been seen with the related arylation of graphene using diazonium compounds. The optical properties of this polyhydrogenated graphene were also further investigated by steady-state as well as by time-resolved femtosecond transient absorption spectroscopy. ${ }^{91}$

Next to the Birch reduction also the protonation of GIGs was investigated as a method for graphene hydrogenation. In order to study the general feasibility of this type of protonation, three different graphite sources, synthetic spherical (SGN18), natural flake (NG) and expended powder (PEX10) were used as starting material. Moreover, the amount of potassium and the nature of the proton/deuterium source were varied in order to obtain deeper insights into the underlying reactivity principles. ${ }^{92}$ The potassium to carbon ratio has been varied between 1:4, 1:8, and 1:24. Three different sources for protons (i.e., $\mathrm{H}_{2} \mathrm{O}$, $\mathrm{MeOH}$, tert- $\mathrm{BuOH})$ as well as the corresponding deuterated analogues have been investigated. The results clearly showed that in this way the bulk synthesis of hydrogenated graphene with tuneable hydrogen content between $5 \%$ and $66 \%$ can be accomplished. In 
addition to the hydrogenation of bulk material, the hydrogenation of flakes deposited on a surface has also been accomplished. ${ }^{92}$ The advantage of the latter method is that the product formation of an entire flake can be investigated using SRM. Both the hydrogenation as well as the deuteration of monolayer graphene was investigated.

For a direct comparison of the outcome of the reductive functionalization of graphene in the bulk versus graphene sheets deposited on a surface, a series of bis-functionalization experiments was carried out (Schemes 3 and 4). ${ }^{93}$ Subsequent addition reactions were done by the treatment of graphenides with diazonium salts and alkly iodides. The addition chemistry of graphenides dispersed in a solvent can in principle take place from both sides of the plane (ditopic) whereas in CVD graphenides supported on a $\mathrm{Si} / \mathrm{SiO}_{2}$ surface attack can only take place single sided (monotopic). For the corresponding bulk functionalisation, pristine natural graphite was exfoliated by wet chemical reduction using $\mathrm{Na} / \mathrm{K}$ alloy in $1,2-$ dimethoxy-ethane (DME). After activation with sodium potassium alloy, the graphenide sheets where treated with the first electrophile. After work up, a second activation with $\mathrm{Na} / \mathrm{K}$ alloy was initiated followed by addition of the second electrophile. As electrophile, 4methoxyphenyl diazonium tetrafluoroborate (A) and hexyl iodide (B) were used. Both combinations of reaction sequences using $\mathrm{A}$ first and $\mathrm{B}$ second and the other way round where systematically applied.

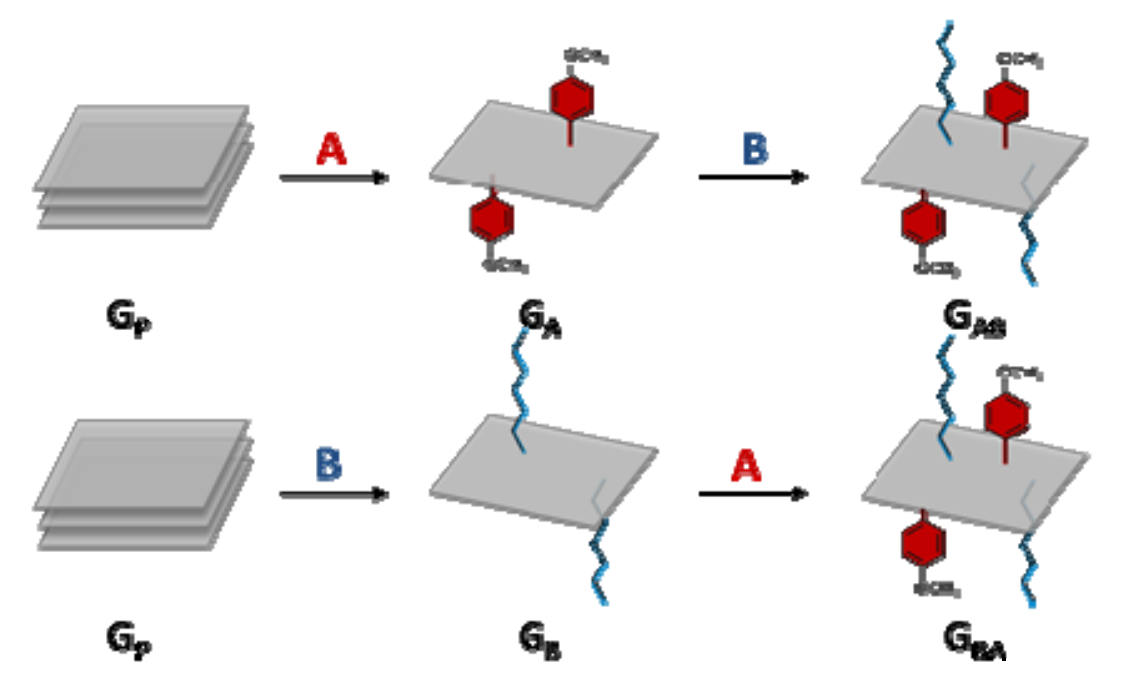

Scheme 3 Schematic representation of the ditopic bisfunctionalization of graphenides in the bulk. Reproduced with permission from ref. 93. Copyright 2016 Wiley-VCH. 
In the case of the bulk functionalization of dispersed graphenide sheets (Scheme 3), after each addition step the degree of functionalization increased as expected. However, in the case of the treatment of the surface supported graphenides the situation was different (Scheme 4). In this case, after the initial addition of hexyl iodide the degree of functionalization could be increased when in the second step the diazonium salt was allowed to react. On the other hand, the degree of functionalization decreased when first the aryldiazonium salt and then the treatment with hexyl iodide was applied. It turned out that in the second case, the retro-reaction, which is stimulated by the charging of the arylated monolayer is the predominant process compared to the subsequent hexylation with hexyl iodide.

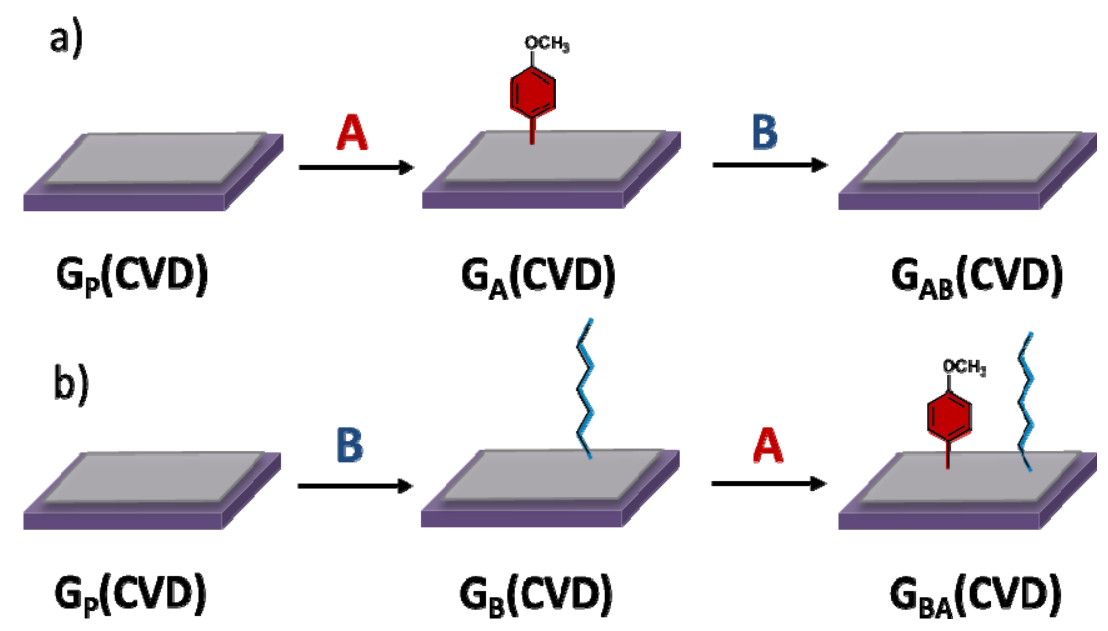

Scheme 4 Schematic representation of the monotopic bisfunctionalization of graphenides supported on a surface. Reproduced with permission from ref. 93. Copyright 2016 Wiley$\mathrm{VCH}$.

It has to be pointed out that related reductive retro-functionalizations were already observed in fullerene and carbon nanotube chemistry. ${ }^{94-96}$ The difference between the two reaction sequences for the surface functionalisation of graphene can be explained by the fact that the aryl anion is the better leaving group compared with the alkyl anion. On the other hand, the difference between the ditopic bulk functionalization and the monotopic functionalization is due to the fact that strain-free addition geometries can be adopted in the former case, whereas in the latter case the increasing degree of addition leads to a decreased 
thermodynamic stability of the adducts. This eventually leads, when sufficiently good leaving groups can be generated, to retro-reactions being preferred.

Another very interesting question with respect to the reductive functionalization of graphene deposited on a surface is whether the reactivity of bilayer and monolayer graphene is different. This problem has been addressed with the identification of a graphene flake consisting of a monolayer region located next to a bilayer region (Fig. 16). ${ }^{97}$
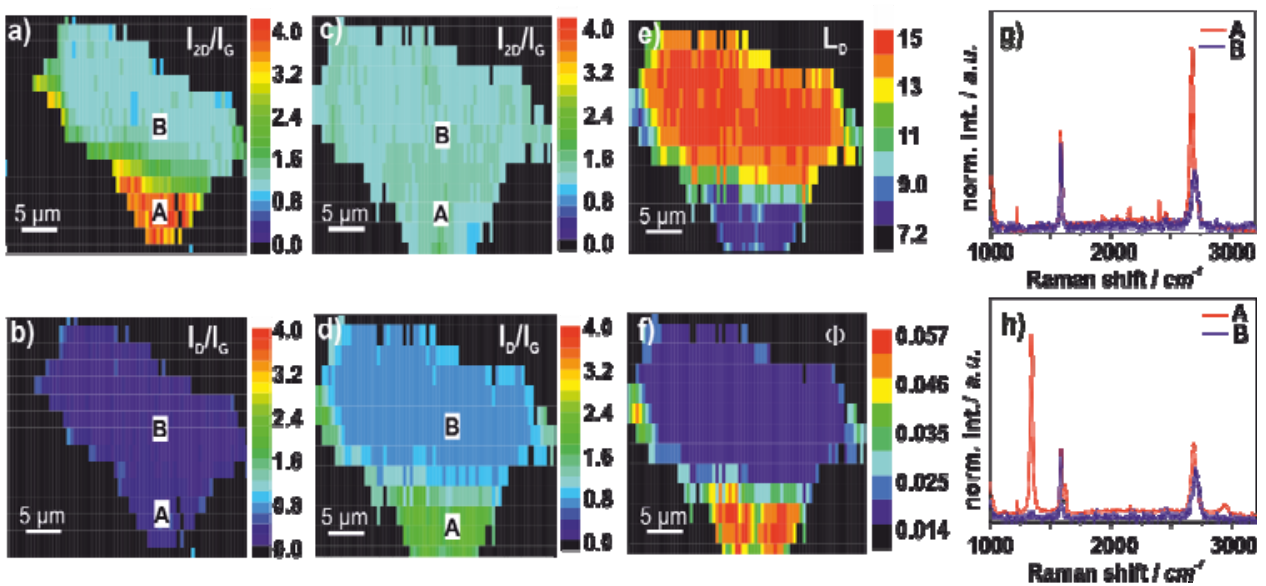

Fig. 16 Scanning Raman microscopy images of an arylated graphene flake (monolayer region A, bilayer region B): $I_{2 D} / I_{G}$ and b) $I_{D} / I_{G}$ map of pristine mechanically exfoliated graphene; c) $I_{2 D} / I_{G}$ and d) $I_{D} / I_{G}$ map of functionalized graphene; e) $L_{D}$ map of functionalized graphene; f) $\Phi$-map of functionalized graphene; g) Raman spectra of monolayer area A and bilayer area B of pristine graphene; h) Raman spectra of monolayer area A and bilayer area B of functionalized graphene. Reproduced with permission from ref. 97. Copyright 2016 Wiley-VCH.

This flake was functionalized by the addition of the blue solution of $\mathrm{NaK}_{3}$ in DME and the subsequent treatment with bis(4-tert-butyl-phenyl)iodonium-hexafluorophosphate (Scheme $5)$.

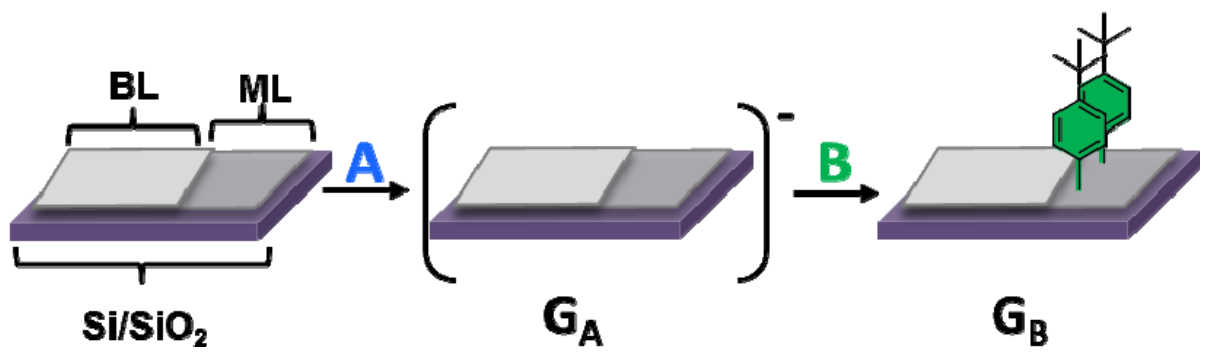


Scheme 5 Schematic representation of the preferred monolayer functionalization of a graphenide flake consisting of both mono- (ML) and bilayer (BL) regions. Reproduced with permission from ref. 97. Copyright 2016 Wiley-VCH.

By carrying out SRS and SRM, it was realized that the monolayer region of this flake exhibited a much higher degree of functionalization (Fig. 16). Obviously, in case of the bilayer graphene, the underlying graphene layer serves as a buffer layer which disfavours multiple outside attacks in the same region. This implies that an attack of an addend to the outer layer is disfavoured because no quenching reaction at the other side can take place. The underlying graphene sheet is too inert to do so. If ditopic quenching cannot take place, the only possibility for quenching the resulting dangling bonds would be a second attack from the same side (monotopic). Such a process however would be associated with an increase of strain energy due to unfavourable bond angles. On the other hand, if an initial attack of an electrophile can be supported by a second attack from a reactive group of the substrate, an almost strain-free binding situation can be accomplished. This is actually guaranteed by the substrate $\mathrm{Si} / \mathrm{SiO}_{2}$ where a lot of surface reactive sites like for example hydroxy groups are present. This assumption was also collaborated by theoretical calculations on monolayer and bilayer graphene on a nickel substrate which also can play the role of a reactive surface being able of forming covalent substrate-graphene bonds. More recently, the covalent functionalization of single and bilayer graphene on $\mathrm{SiO}_{2} / \mathrm{Si}$ was effected through sequential treatment with the alkalide reductant $\left[\mathrm{K}(15 \text {-crown-5 })_{2}\right] \mathrm{Na}$ and electrophilic aryl or alkyl halides, using Raman and X-ray photoelectron spectroscopies to evaluate the degrees and uniformity of the functionalization. ${ }^{98}$ Interestingly, a study on the reactivity of isotopically labelled bilayer graphene indicates the functionalization of both layers, in a mechanism which may involve the diffusion and/or intercalation of the reactants between the graphitic layers.

A very important question that arises with respect to the chemistry of graphenides is what happens if GICs or graphenides deposited on surfaces are exposed to ambient conditions such as oxygen and water. In this regard recently a very important discovery was made. ${ }^{99}$ It was found that reduced graphites such as GICs, graphite dispersions and graphenides deposited on surfaces can by quantitatively discharged by the simple treatment with 
benzonitrile. This is because of its comparatively low reduction potential which allows for the easy formation of the corresponding radical anion which exhibits a red colour and can serve as a reporter molecule for the quantitative determination of the negative charges. This discovery has a variety of consequences, namely a quantitative solvent-induced reduction and electrostatically driven mass transport of $\mathrm{K}^{+}$from the GICs into the liquid. On the other hand, the simple treatment of dispersed graphenides suspended on silica substrates leads to the clean conversion to graphene. If on the other hand such graphenide deposits are not treated with benzonitrile and are exposed to ambient conditions, it was observed that hydrogenation and hydroxylation of the graphenides takes place. The latter side reaction can also be considered as a reductive functionalization of graphene.

\section{Non-covalent functionalization of graphene}

\subsection{Sonication-induced, liquid-phase exfoliation of graphene}

The liquid phase exfoliation of graphene typically involves three steps: i) exfoliation of graphite, ii) stabilization of the graphene sheets, and iii) separation from the bulk graphitic starting material (usually by centrifugation). Although van der Waals forces are classified as weak attractive interactions, they play a major role in graphite maintaining the graphene layers stacked following an $\mathrm{AB}$ or Bernal-stacked pattern structure (Fig. 1b). ${ }^{50}$ Thus, the successful exfoliation of graphite towards graphene necessarily should overcome the van der Waals attraction between adjacent layers by applying external physical forces. In this regard, the mechanical liquid phase exfoliation of graphite combines a liquid immersion that diminishes the strength of the van der Waals interactions and allows the liquid to slide between sheets favouring their separation, and an ultrasonication process, as exfoliation strategy (Fig. 17a). The microscopic gas bubbles that usually exist in the liquid are submitted to an acoustic field (sound energy of frequencies $<20 \mathrm{KHz}$ ), oscillating and growing in size until they rapidly collapse by a pressure increase. This physical phenomenon called cavitation, induces compressive stress to the bulk graphite, and overcome the van der Waals forces resulting in exfoliation. ${ }^{100,101}$ 
a)

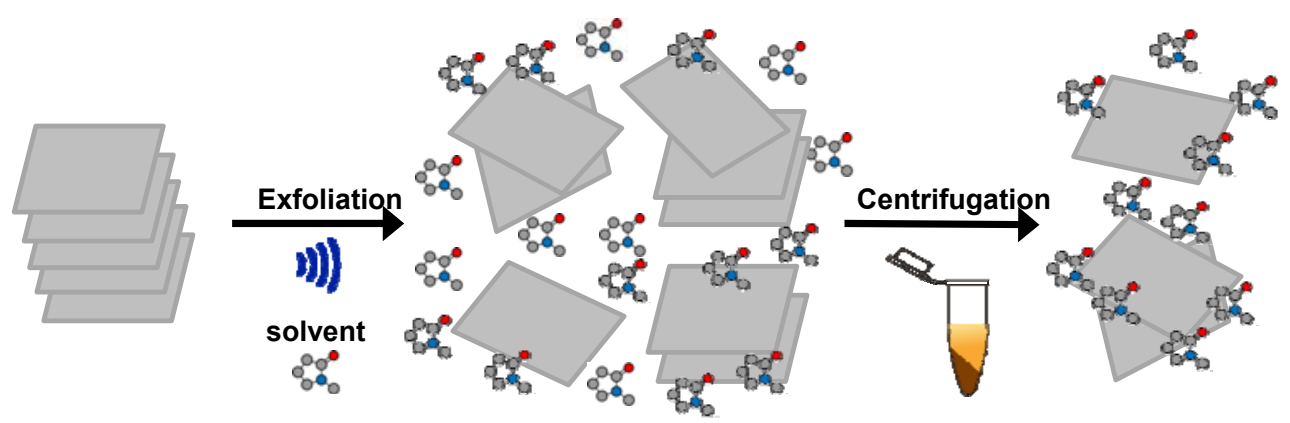

b)

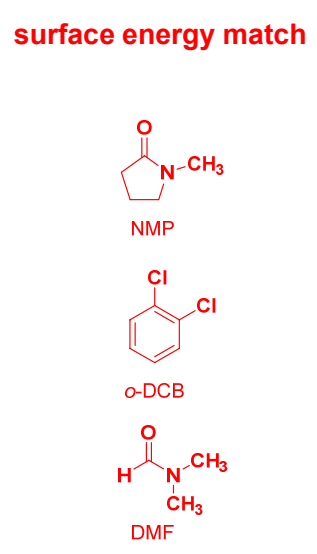

charge transfer contribution
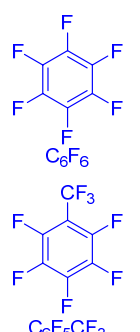

$\stackrel{\mathrm{F}}{\mathrm{C}_{6} \mathrm{~F}_{5} \mathrm{CF}_{3}}$

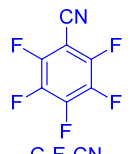

$\mathrm{C}_{6} \mathrm{~F}_{5} \mathrm{CN}$

(F)
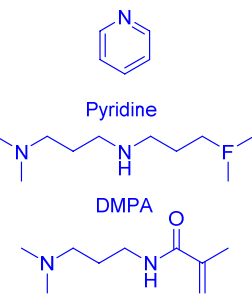

DMAPMA

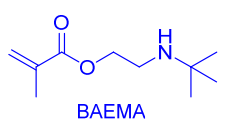

$\overbrace{\text { MAEMA }}^{O} \overbrace{}^{\prime}$
Ionic Liquids
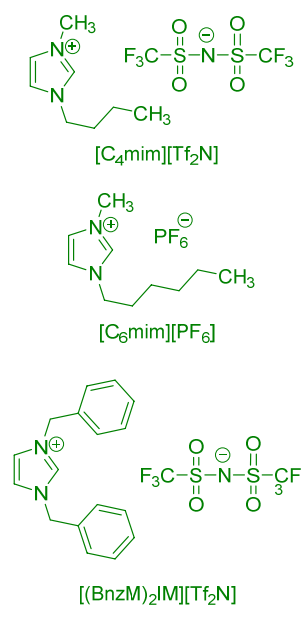

Fig. 17 a) Schematic representation of the sonication-induced, liquid-phase exfoliation of graphite using NMP as representative solvent. b) Chemical structures of solvents and ionic liquids used in graphene exfoliation and discussed in the text.

It has been demonstrated that the liquid phase exfoliation of graphite can only take place through a small energetic cost (mixing enthalpy per unit volume). ${ }^{102}$ Coleman and coworkers realized that closer surface energies between graphene and solvent diminish the mixing enthalpy favouring exfoliation. They tested graphite exfoliation in a wide range of solvents, measuring the resulting concentration of the graphene dispersions after ultrasonication and centrifugation using UV-vis spectroscopy. The best performances were obtained for low viscosity solvents, with a surface tension $(\gamma)$ of $40-50 \mathrm{~mJ} / \mathrm{m}^{2}$ at room temperature and for short sonication times (long or intensive sonication periods can cause the solvent degradation, therefore changing its properties). ${ }^{100}$ The most popular candidates 
for the liquid exfoliation protocol are $N$-methyl pyrrolidone (NMP, $\gamma=40 \mathrm{~mJ} / \mathrm{m}^{2}$ ) (in a recent report used in combination with $\alpha$ functionalized alkanes), ${ }^{103} o$-DCB $(\gamma=37$ $\mathrm{mJ} / \mathrm{m}^{2}$ ), and $N, N$-dimethylformamide (DMF, $\gamma=37.1 \mathrm{~mJ} / \mathrm{m}^{2}$ ), which all present surface energy values within the previously mentioned range. ${ }^{102}$ However, even when the sonication protocol produces pristine graphene of high quality and in amounts suitable for laboratory experiments, the concentration of the graphene dispersions obtained do not exceed $0.01 \mathrm{mg} / \mathrm{mL}$, which is unfeasible for applications in the industrial field. Alternative strategies that have been explored to increase the graphene's concentration, while maintaining the quality of the flakes, are: i) the increase of the sonication time up to 460 hours, which provides highly concentrated graphene dispersions $(1.2 \mathrm{mg} / \mathrm{mL}),{ }^{104}$ ii) shear mixing, ${ }^{105}$ or iii) tip instead of bath sonication. ${ }^{106,107}$ However, all the mentioned processes reduce the flake size and induce edges defects. Moreover, the high boiling point solvents used are difficult to be removed which is detrimental in terms of practical purposes.

Besides ultrasonication, a mechanical stimulus such as the ball-milling treatment has been also recently employed to exfoliate graphite through interactions with commercially available melamine under solid conditions. ${ }^{108}$ This procedure allows the fast production of relatively large quantities of material with a low presence of defects.

The use of mixed solvents has been also pursued as an alternative strategy for the exfoliation of 2-D materials - not only graphene but also $\mathrm{MoS}_{2}, \mathrm{WS}_{2}, \mathrm{~h}-\mathrm{BN}, \mathrm{Bi}_{2} \mathrm{Se}_{3}$, $\mathrm{MoSe}_{2}, \mathrm{SnS}_{2}$, and $\mathrm{TaS}_{2}$ - combining two "mediocre" solvents that form strong cosolvents. ${ }^{109,110}$ This approach incorporates several interesting advantages: i) avoids the health damage that the high toxicity of the typically used NMP, $o$-DCB and DMF solvents can produce, by replacing them with environmentally-friendly solvents (alcohols, acetone and water) that attain similar surface tension properties; ii) reduces the costs by using common co-solvents instead of more expensive pure solvents, and iii) provides a large library of co-solvents by multiple combinations. In particular, water/acetone mixtures achieve graphene dispersions composed of around a $50 \%$ of thin nanosheets, concentrations up to $0.21 \mathrm{mg} / \mathrm{mL}$, and free of basal plane defects or oxygenated groups. ${ }^{111}$

Besides physical forces like the surface tension, charge transfer phenomena also play an important role in liquid phase exfoliation of graphene. A research study published in 2009 by Bourlinos, Georgakilas et al. showed an enhancement on graphene dispersions 
concentration (between $0.05-0.10 \mathrm{mg} / \mathrm{mL}$ ) while exfoliating graphite with aromatic electron-accepting solvents, such as hexafluorobenzene, octafluorotoluene, pentafluorobenzonitrile, and pentafluoropyridine (Fig. 17b). ${ }^{112}$ The electron-withdrawing solvents stabilize the electron-rich graphene in a charge transfer phenomena through $\pi-\pi$ stacking. Furthermore, the exfoliation capability of each solvent is related with its electronic nature. Control experiments using non-fluorinated analogues such as benzene, toluene and nitrobenzene, reveal little effectiveness in terms of stabilization of the graphene dispersions. Following the same strategy, when mixing hexafluorobenzene with benzene in an equimolar ratio, graphene dispersions with very high concentrations - up to $50 \mathrm{mg} / \mathrm{mL}$ were obtained. ${ }^{113}$ The situation was markedly different when using pyridine as exfoliating solvent, where dispersions of concentrations about $0.3 \mathrm{mg} / \mathrm{mL}$ and stable for a week were obtained. ${ }^{112}$ The pyridine case suggests that aromatic donors may also exfoliate graphite in the reverse way, that is, charge transfer through $\pi-\pi$ stacking from the solvent molecules to graphene, forcing the latter to act as an electron-withdrawing species. In this sense, other nitrogen-based organic solvents also provided colloidal dispersions of graphene. Significant examples are the direct exfoliation of graphite with 3,3'-iminobis $(N, N$ dimethylpropylamine) (DMPA), $\quad N$-[3-dimethylamino)propyl]methacrylamide (DMAPMA), 2-(tert-butylamino)ethyl methacrylate (BAEMA) and 2(dimethylamino)ethyl methacrylate (MAEMA). An extremely high concentration $(\sim 15$ $\mathrm{mg} / \mathrm{mL}$ ) was achieved and approximately $98 \%$ of the obtained graphene remained dispersed, without sedimentation, over long periods of time. ${ }^{114}$

Ionic liquids (ILs) have also been employed as dispersing and stabilizing media in graphene exfoliation. From the initial trial of Dai et al., ${ }^{115}$ where pristine graphene nanosheets were obtained by direct ultrasound exfoliation of graphite flakes in a conventional IL, 1-butyl-3methylimidazolium bis(trifluoromethanesulfonyl)-amide $\quad\left[\mathrm{C}_{4} \mathrm{mim}\right]\left[\mathrm{Tf}_{2} \mathrm{~N}\right]$, with concentrations of $0.95 \mathrm{mg} / \mathrm{mL}$, other examples have been reported with increasing yields of exfoliated graphene in 1-hexyl-3- methylimidazolium hexafluorophosphate $\left[\mathrm{C}_{6} \mathrm{mim}\right]\left[\mathrm{PF}_{6}\right]$ $(5.33 \mathrm{mg} / \mathrm{mL})^{116}$ or 1,3-bis(phenylmethyl)imidazolium bis(trifluoromethanesulfonyl)amide $\left[(\mathrm{BnzM})_{2} \mathrm{IM}\right]\left[\mathrm{Tf}_{2} \mathrm{~N}\right](5.8 \mathrm{mg} / \mathrm{mL}) .{ }^{117}$ One of the most striking investigations carried out with ILs is a recent work published by Aida and co-workers, ${ }^{118}$ based on a microwaveassisted exfoliation of graphite with molecularly-engineered oligomeric ILs, almost 
quantitative yields (93\%) were obtained, with a high selectivity (95\%) towards single layer graphene. The authors claim that this technique is able to exfoliate graphite in 30 minutes with an excellent structural integrity of the graphene flakes obtained.

\subsection{Non-covalent functionalization of graphene in solution}

Dispersions of graphene in water have been obtained with classical surfactant stabilizers such as sodium dodecylbenzenesulfonate (SDBS), sodium cholate (SC) or cetyltrimethylammonium bromide (CTAB), where the dispersed graphene flakes are stabilized against re-aggregation by Coulomb repulsion due to the adsorbed surfactant. Coleman and co-workers pioneered this method to disperse and exfoliate graphite to give graphene suspended in water-SDBS solutions. ${ }^{119}$ By means of TEM, they demonstrated that the dispersed phase consists of small graphitic flakes, which are monolayer $(\sim 3 \%)$ and $<5$ layers (40\%). In addition, atomic resolution TEM showed the monolayers to be, on average, free of defects. In further investigations, graphene dispersions of up to $0.3 \mathrm{mg} / \mathrm{mL}$ were obtained in water with the aid of SC. Detailed TEM analysis showed that the flakes consist of 1-10 stacked monolayers with up to $20 \%$ of the material containing just one layer. The average flake consists of $\sim 4$ stacked graphene layers and has a length and width of $\sim 1 \mu \mathrm{m}$ and $\sim 400 \mathrm{~nm}$, respectively. However, the mean flake length decreases with increasing centrifugation rates. ${ }^{120}$ The ultrasound-induced, liquid phase exfoliation was employed to directly exfoliate graphene in DMF from graphite and using CTAB as surfactant. ${ }^{121}$ Characterization of the flakes by UV-vis spectroscopy, SEM, TEM, AFM and Raman spectroscopy showed the successful exfoliation into graphene flakes of average thickness $\sim 1.2 \mathrm{~nm}$.

Stable dispersions of graphene sheets in water and organic solvents can also be obtained through non-covalent functionalization with a wide range of polymers. ${ }^{26}$ The combination of both materials results in hybrids with improved mechanical strength, flexibility, or tuneable electro-optical properties by doping. Different non-ionic and ionic polymers have been used to disperse and stabilize graphene and, in general, it has been observed that nonionic polymers significantly outperform their ionic counterparts. However, more recent investigations considering three polysaccharides with different electrostatic nature: nonionic pullulan, cationic chitosan and anionic alginate, demonstrated that the different surface free energy and thermodynamic affinity also play a major role in the stabilization of 
the graphene sheets. ${ }^{122}$ Graphene aqueous dispersions, with concentrations of up to 2.3 and $5.5 \mathrm{mg} / \mathrm{mL}$ in pullulan and chitosan solutions, respectively, were achieved, whereas alginate barely interacts with graphene. In any case, the strong polymer/graphene interactions make extremely difficult to separate the produced composites, which in some cases resulted in interesting materials. In this connection, Yoon and co-workers produced stable graphene dispersions by simply sonicating graphite with four different polymers based on either poly(vinyl alcohol) (PVA) or dextran. ${ }^{123}$ The water-soluble polymers with phenyl- and pyrenyl-functionalized side chains facilitated the formation of stable aqueous dispersions of graphene without degrading its $\mathrm{sp}^{2}$ hybridized structure. From the aqueous dispersion of the graphene/polymer it is possible to prepare hydrogels and aerogels. By cross-linking the dispersed polymers in the solution, hydrogels with embedded graphene flakes inside the polymer chain networks are obtained. The subsequent freeze-drying of the hydrogel resulted in an aerogel (Fig. 18a). The variety of possible applications for graphene/polymer gels was demonstrated in two examples where the composite acts as dye adsorbent or gel electrolyte.

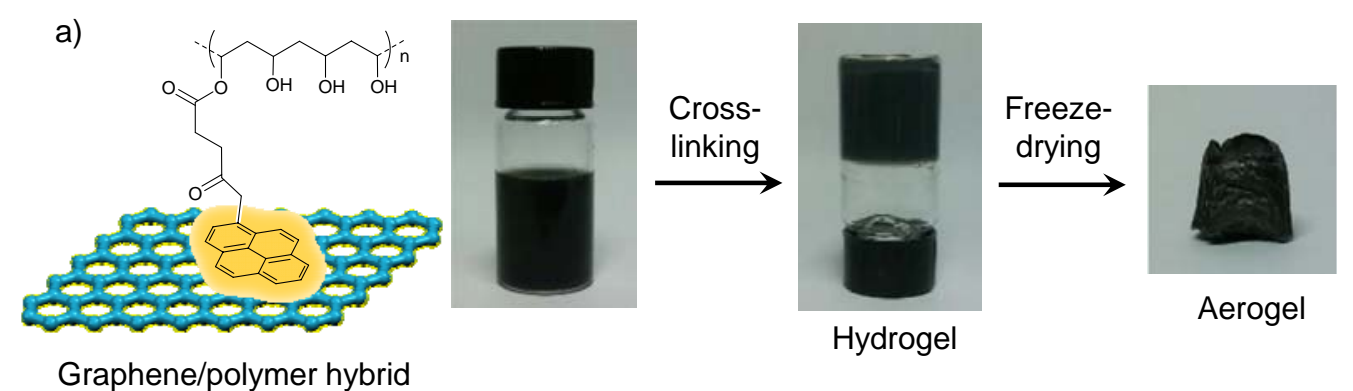

b)
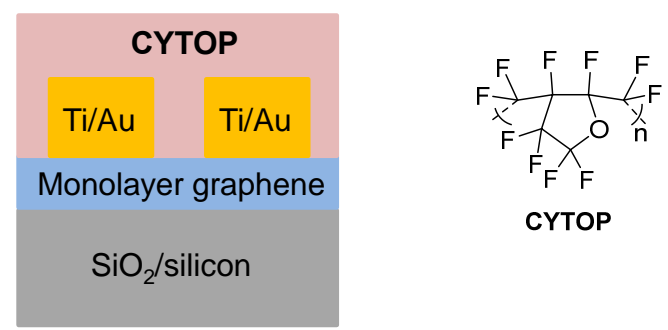

Fig. 18 a) Molecular structure of graphene/pyrene-PVA hybrid (left) and photographs describing the sequential preparation of a hydrogel and an aerogel from an aqueous dispersion of graphene/pyrene-PVA (right). Reproduced with permission from ref. 123. 
Copyright 2015 American Chemical Society. b) Schematic cross-section of a monolayer graphene FET and chemical structure of CYTOP.

The non-covalent interactions of electroactive polymers with graphene can cause the doping by charge transfer processes and the modulation of graphene's work function. This approach has been extensively investigated to improve the performance of graphene-based electronic devices. As a model example, Ruoff and co-workers developed an approach to dope monolayer graphene field effect transistors (FETs) by employing the fluoropolymer poly(perfluoroalkenylvinyl ether) (CYTOP), which resulted in an improvement of the onoff current ratio from 5 to 10 , as well as an increase of the field-effect mobility by as much as a factor of two compared to plain graphene FETs (Fig. 18b). ${ }^{124}$

Decorating graphene with conjugated compounds can be used to obtain stable suspensions of graphene sheets. In particular, PAHs have resulted very useful in this regard due to their $\pi-\pi$ stacking interactions with graphene. In this context, frequently used anchoring groups are naphthalene, anthracene, pyrene, coronene, Pors and their derivatives. ${ }^{26}$ However, the supramolecular hybrids that these compounds form with graphene are often unstable in solvents where the molecular binding groups are highly soluble.

Considering these stability aspects, Dichtel and co-workers designed a multivalent tripod capable of binding graphene through three pyrene units, which enhanced the overall stability. ${ }^{25}$ A Co(II) bis-terpyridyl complex $\left(\left[\mathrm{Co}_{-t p y}\right]^{2+}\right)$ (TPyrCo) was, for example, incorporated onto the graphene-immobilized tripod (Fig. 19). The TPyrCo binding constant and its surface coverage were measured through electrochemical detection of the $\mathrm{Co}^{2+/ 3+}$ redox couple and compared to the monopod. Interestingly, the binding energies of the monopod and the tripod are similar with $38.3 \pm 0.5$ and $38.8 \pm 0.2 \mathrm{~kJ} \mathrm{~mol}^{-1}$, respectively. The authors postulated that the comparable values for the different systems could be caused by the energetic cost for the disruption of the non-covalent interaction among the pyrene rings upon binding to graphene. In a further study, the tripodal receptors were modified to anchor proteins relevant to graphene biosensors (TPyrNHS) (Fig. 19). The binding specificity of an anti E. coli antibody depends strongly on the nature of the graphene functionalization method. The antibodies retain their specificity when conjugated to tripodal receptors, but exhibit poor $E$. coli cell recognition when immobilized onto the 
monovalent binding group or bare graphene. The same anchoring unit has been recently employed by D'Souza and co-workers for the design of multimodular D-A conjugates, composed of three molecules of pyrene, a subphthalocyanine ( $\mathrm{SubPc}$ ), and a fullerene $\left((\mathbf{P y r})_{3}\right.$ SubPc-C $\mathbf{C}_{\mathbf{6 0}}$ ) (Fig. 19). ${ }^{125}$ The measured redox potentials revealed the influence of graphene on the pyrene and SubPc units, leaving $\mathrm{C}_{60}$ unperturbed because of its distant position from the graphene surface.

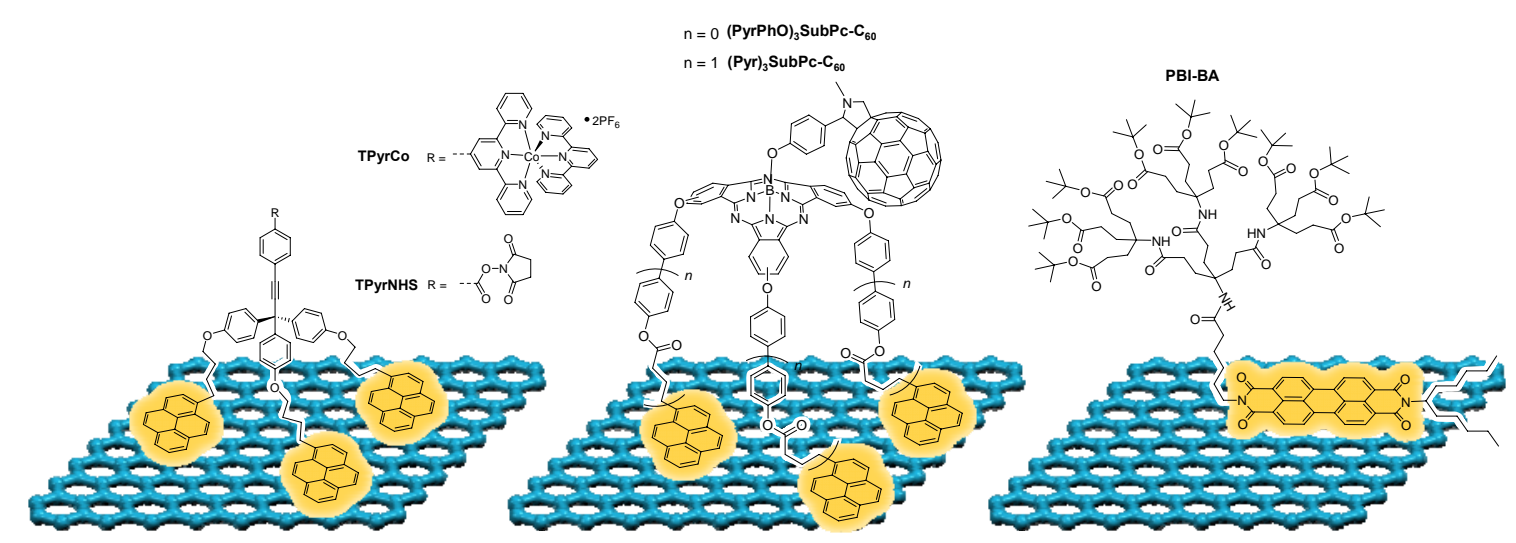

Fig. 19 Representative examples of graphene-based hybrids with tripodal pyrene derivatives (TPyrCo, TPyrNHS, (Pyr) $)_{3}$ SubPc-C 60 and (PyrPhO) $)_{3}$ SubPc-C 60 ) (left and middle) and perylene bisimide bolaamphiphiles (PBI-BA) (right).

The use of multivalent scaffolds for the supramolecular interaction with graphene was also considered by Martín et al. ${ }^{126}$ in the supramolecular modification of graphene with the geometrically mismatched, curved 9,10-di(1,3-dithiole-2-ylidene)-9,10 dihydroanthracene (exTTF) molecule. In this case, gold nanoparticle (AuNP) scaffolds, where multiple units of exTTF are linked, allowed magnifying the exTTF-graphene $\pi-\pi$ and $\mathrm{CH}-\pi$ interactions (Fig. 20).
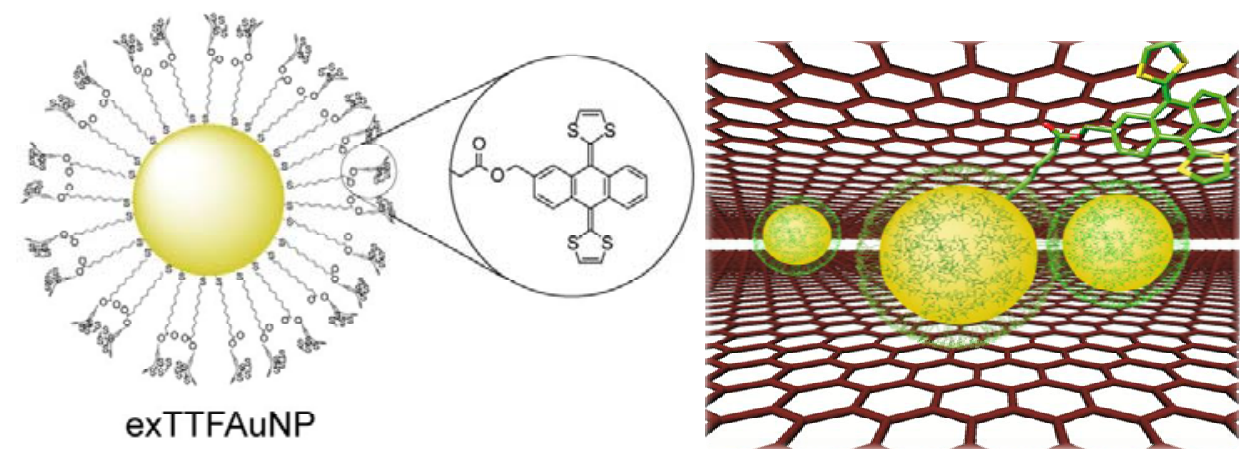
Fig. 20 Representation of a gold nanoparticle endowed with multiple exTTF units (exTTFAuNP) (left) and the resulting graphene-exTTFAuNP conjugates forming sandwich-type nanostructures (right). Adapted with permission from ref. 126. Copyright 2013 Wiley-VCH.

Perylene bisimides (PBIs) are attractive PAHs, which besides being well-suited for $\pi-\pi$ stacking interactions are powerful chromophores. Hirsch and co-workers demonstrated that PBI-based bolaamphiphiles (PBI-BA) (Fig. 19) exhibit a remarkable stability in its interaction with graphene. They can delaminate and stabilize graphene with a flake size of about $1 \mu \mathrm{m}$ and a moderate defect density, of approximately a $0.01 \%{ }^{127}$ Although PBIs exhibit strong fluorescence, no emission is present in the Raman spectrum of a dispersion in NMP of turbostratic graphite with the dendronized PBI, which indicates a pronounced quenching due to the electronic communication with graphene.

\subsection{Non-covalent functionalization of graphene on solid surfaces}

In general, the coating of solid surfaces, namely metals, with atoms, (organic) molecules, nanoparticles and polymers is an innovative area that has received the attention of the scientific community along the last recent years. This is particularly interesting when considering graphene since the presence of more or less active metal atoms, can significantly modify the electronic properties of graphene, resulting in new materials with enhanced or new properties of interest in the preparation of devices for different purposes. $^{128}$

In this regard, the coverage of solid surfaces, namely active metals such as $\mathrm{Au}, \mathrm{Cu}$, or $\mathrm{Ag}$ or semimetals like graphite, requires the detailed understanding of the phenomena occurring at the atomic scale. More precisely, at the interface between the solid surface and the added (organic) molecules. ${ }^{129-131}$ However, despite fascinating studies have been carried out on different solid substrates with a large number of (organic) molecules, this is not the case when pristine graphene is considered to interact with them. The controlled coverage of solid surfaces by chemical species needs from new tools, namely microscopy techniques (STM, AFM, and TEM), and a precise understanding of the electronic interactions occurring between the substrate and the adsorbed chemical systems as well as those interactions existing between the deposited molecules in the presence of the chemically 
non-innocent solid surface. Actually, this study paves the way to graphene as a reactive surface and to a new and less-explored 2-D supramolecular chemistry. ${ }^{132}$

Graphene grown on metallic surfaces forms a moiré superlattice showing a singular corrugation on the graphene surface with structural and electronic modifications when compared to pristine graphene. The impact of this corrugated graphene surface on the molecular deposition of chemical species is strongly dependent on the nature of the underlying metal substrate. Thus, whereas some metals such as $\operatorname{Ir}(111)$ have a weak electronic interaction with the deposited chemical species, others such as $\operatorname{Ru}(0001)$ and $\mathrm{Ni}(111)$ have a strong electronic communication. ${ }^{133,134}$ It is interesting to note that most of the molecular self-assemblies on metal surfaces are performed under ultra-high vacuum conditions to ensure the best experimental conditions.

Although some studies on depositing molecules on graphene grown on metallic surfaces have been reported in the literature, we will discuss just a few examples to illustrate the interaction between electroactive molecules and the epitaxially grown graphene on different metal surfaces affording doped graphene.

An interesting example showing the influence of moiré corrugation is that of the adsorption of 2,4'-bis(terpyridine) (2,4'-BTP, Fig. 21a) on graphene. The molecule form 2-D supramolecular arrays due to the formation of $\mathrm{C}-\mathrm{H} \cdots \mathrm{N}$ H-bonding (Fig. 21b). ${ }^{135}$ Interestingly, all the molecules were located in the lower part of the corrugated surface (valleys) remaining the upper parts (hills) mostly free (Fig. 21c). Again, the strong influence of the underlying metal surface is responsible for the observed findings since when using $\mathrm{Ag}$ as a base substrate, highly ordered 2-D monolayers were formed. ${ }^{135}$
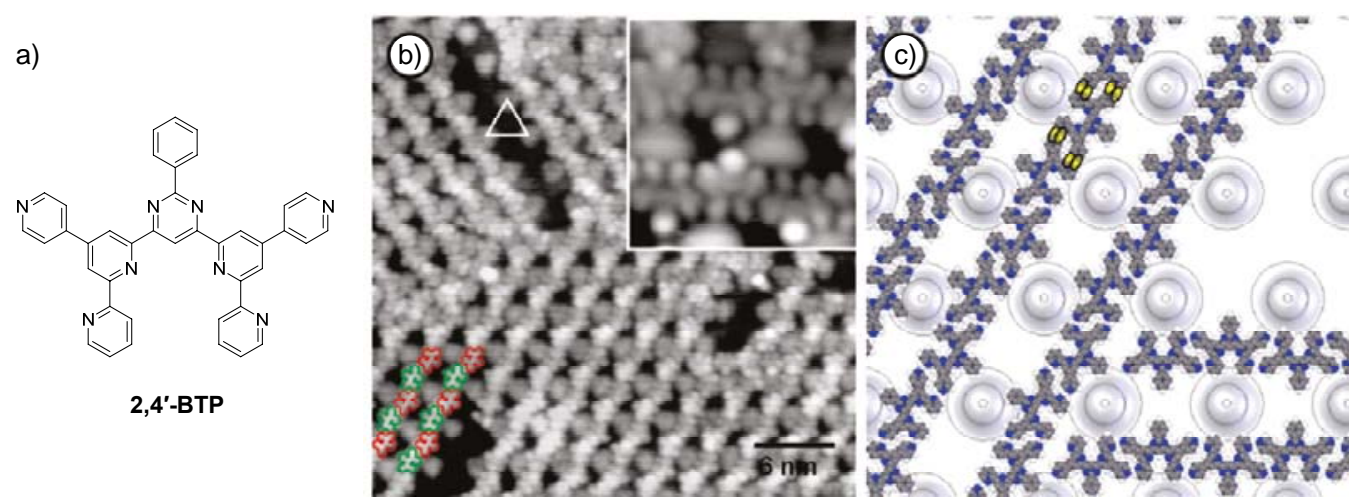

Fig. 21 a) Molecular structure of 2,4'-BTP. b) STM image of an adlayer of 2,4'-BTP molecules on graphene $/ \mathrm{Ru}(0001)$ at $\mathrm{T}=115 \mathrm{~K}$. The antiparallel arrangement of the 
molecules is indicated by alternating colors. Inset: detail of the structure at $\mathrm{T}=130 \mathrm{~K}$. c) True to scale model of $\mathbf{2 , 4}$ '-BTP admolecules on graphene with hydrogen bonding configuration (double hydrogen bonds, yellow ellipses). Reproduced with permission from ref. 135. Copyright 2011 American Chemical Society.

Oligothiophenes, namely QT and QTDA (Fig. 22a) have been deposited on CVD-grown graphene on copper foil by using STM under ambient conditions. Remarkably, QT molecules form large domains, in the range of hundreds of nanometers, showing lamellae features mostly covering the underlying mono- and multi-atomic steps. Similarly, QTDA molecules form smaller domains in a preferentially lamellar array. However, in contrast to the QT, these domains span over the step edges as well as terraces of the underlying copper surface (Fig. 22b-g). It is interesting to notice that although $\mathrm{Cu}(111)$ and $\mathrm{Cu}(100)$ facets dominate the surface of polycrystalline copper after annealing, the authors reported to get identical supramolecular assemblies regardless of the facets nature. ${ }^{136}$

a)
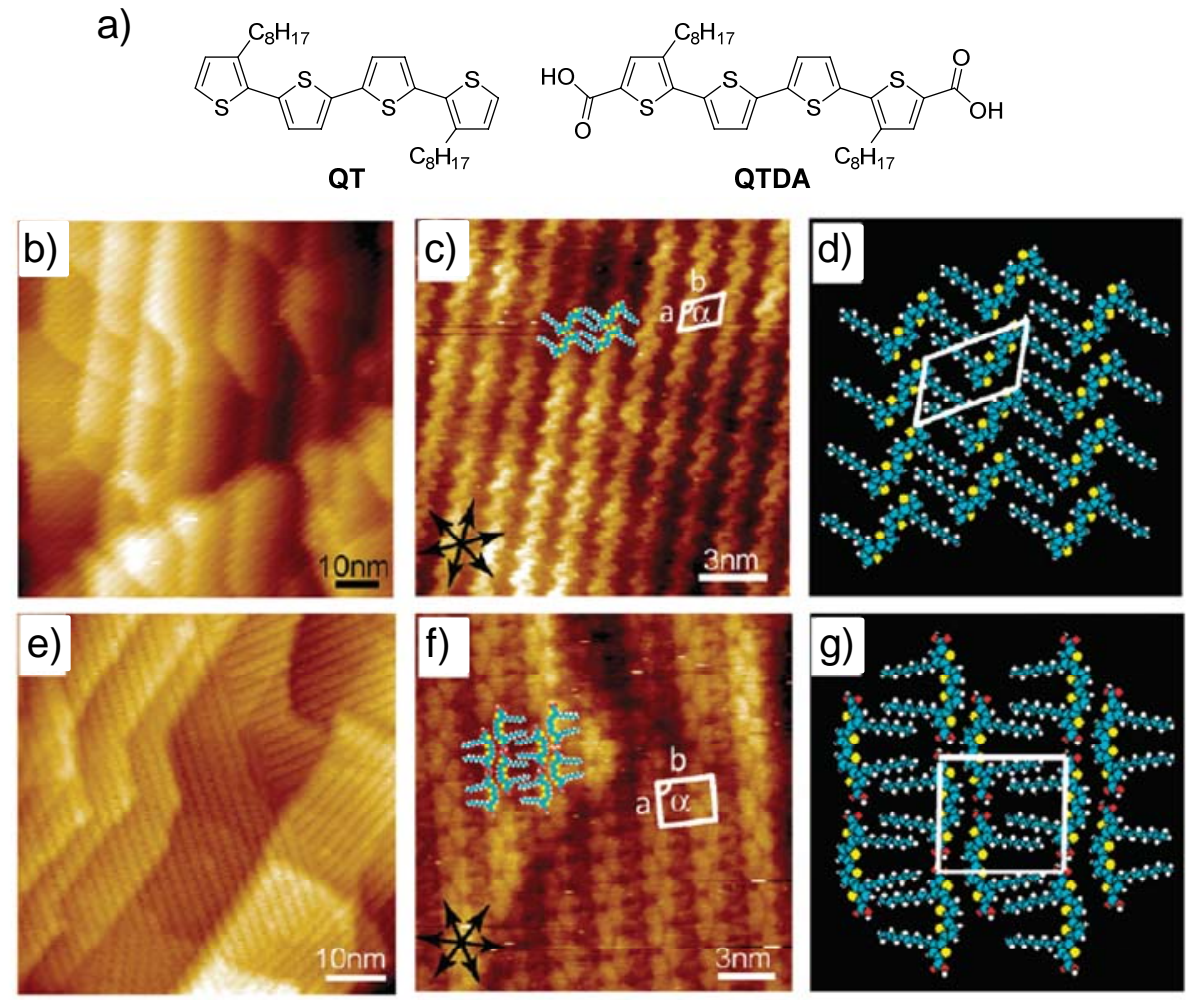

Fig. 22 a) Molecular structure of QT and QTDA. Large scale and high resolution STM images of the (b, c) QT and (e, f) QTDA adlayer on G-copper.. d) and g) are the tentative 
models of the QT and QTDA assembly structures, respectively. The black stars in the lower left corner of the c) and f) STM images indicate the orientation of the graphene lattices. The orientation of the step edges in b) and e) indicates that graphene is grown on the $\mathrm{Cu}(111)$ facet. Reproduced with permission from ref. 136. Copyright 2013 Royal Society of Chemistry.

Remarkable examples are the electron acceptor molecules 7,7,8,8-tetracyano- $p$ quinodimethane (TCNQ) and 2,3,5,6-tetrafluoro-7,7,8,8-tetracyano-p-quinodimethane $\left(\mathrm{F}_{4}\right.$ TCNQ) affording hole doped graphene. ${ }^{137}$ Both molecules exhibit the same molecular geometry but with a different chemical behaviour. In TCNQ, the four hydrogen atoms on the quinonoid moiety are able to form a net of hydrogen bonding leading to the formation of a compact 2-D self-organized monolayer. Furthermore, in contrast to the negatively charged four fluorine atoms in $\mathrm{F}_{4}$-TCNQ, these hydrogen atoms show a positive electrostatic potential, thus interacting intermolecularly with the cyano groups of a neighbour molecule. The single monolayer of epitaxially grown graphene on $\operatorname{Ir}(111)$ electronically decouples the adsorbed electron acceptor molecules from the metallic substrate, thus allowing the study of their 2-D supramolecular self-organization (Fig. 23a,b). STM imaging of HOMO and LUMO orbitals are similar to those of the pristine TCNQ molecule, which indicates a low degree of charge transfer from the graphene. The repulsion between the fluorine atoms in $\mathrm{F}_{4} \mathrm{TCNQ}$ leads to a less compact organization with freely rotating molecules (Fig. 23c,d).
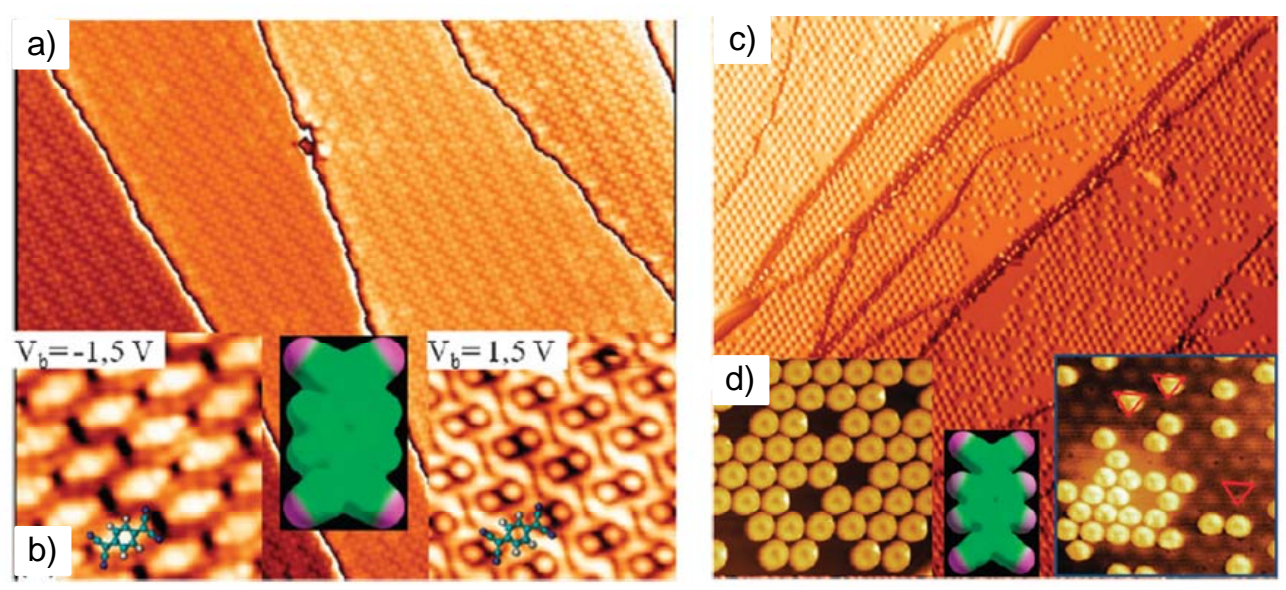
Fig. 23 a) STM image taken at $4.6 \mathrm{~K}$ after depositing TCNQ on graphene/Ir(111). b) STM images taken at $-1.5 \mathrm{~V}$ (left panel) and $+1.5 \mathrm{~V}$ (right panel). In both panels, a ball-and-stick model of the TCNQ molecule has been superimposed to show the molecular orientation. The central panel shows the electrostatic potential map of TCNQ (purple for negative and green for positive of TCNQ). c) STM image taken at $77 \mathrm{~K}$ after depositing $\mathrm{F}_{4}$ TCNQ on graphene/Ir(111). d) STM images taken at $-1.0 \mathrm{~V}$ (left panel) and $-0.05 \mathrm{~V}$ (right panel). Both images were recorded at $77 \mathrm{~K}$. Central panel shows electrostatic potential map of $\mathrm{T}_{4} \mathrm{TCNQ}$ (purple for negative and green for positive). Reproduced with permission from ref. 137. Copyright 2010 Royal Society of Chemistry.

In contrast to the aforementioned study involving $\operatorname{Ir}(111)$, deposition on an epitaxial buffer layer of graphene grown on $\mathrm{Ru}(0001)$ affords TCNQ molecules exhibiting a magnetic moment which induces the formation of ferromagnetic order in the 2-D molecular arrangement (Fig. 24). ${ }^{138}$ This magnetic moment stems from the charge transferred from the graphene $/ \mathrm{Ru}(0001)$ to the electron accepting TCNQ molecule. The epitaxial graphene layer is $n$-doped on $\mathrm{Ru}(0001)$, and it is further transferred to the TCNQ acceptor molecule deposited on the graphene layer. Actually, approximately one electron is transferred leading to a singly occupied orbital. This unpaired electron leads to a small but persistent magnetic moment of around $0.23 \mu \mathrm{B}$ per molecule. Surprisingly, the magnetic moment survives even when two or more TCNQ molecules couple together, which is a requirement for getting a magnetic order. Despite the deposition is monitored at low temperatures $(4.6 \mathrm{~K})$ and occurs on small areas of the surface, experimental evidences confirm both the existence of a small magnetic moment (scanning tunnelling spectroscopy, STS) as well as the formation of magnetic domains (STM). Furthermore, spin-polarized calculations for the TCNQ monolayer on graphene/ $\mathrm{Ru}(0001)$ predict that the magnetically ordered structure is energetically favoured. The total calculated magnetic moment per unit cell (containing 8 molecules) is $1.3 \mu \mathrm{B}$ which represents an average magnetic moment of $0.18 \mu \mathrm{B}$ per molecule. An analysis of the spin density shows that this magnetic moment stems from the unpaired electrons in the SOMO-derived band.

Although the reported results show the formation of magnetic domains, the mechanism that ferromagnetically couples the local moments to each other is complex provided that it 
involves simultaneously several chemical systems, namely the electron acceptor TCNQ molecules, the graphene layer and the ruthenium substrate. These results, however, open a new scenario in the use of graphene for further electronic applications since they show that other pathways in organic electronics and spintronics are possible. In particular, those aspects related with spin-filters or 2-D spin polarizers where the properties of graphene need to be skilfully combined with the appropriate magnetic properties.

a)
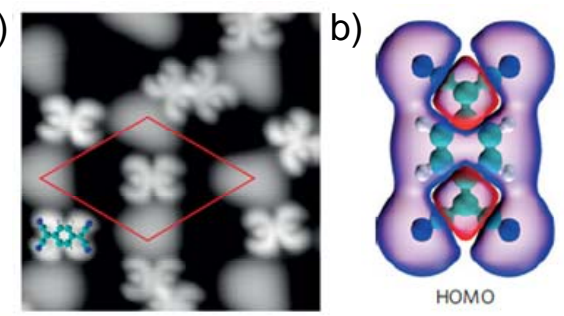

номо

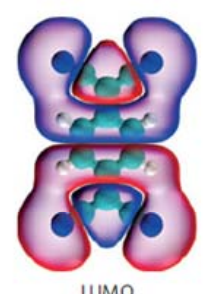

c)
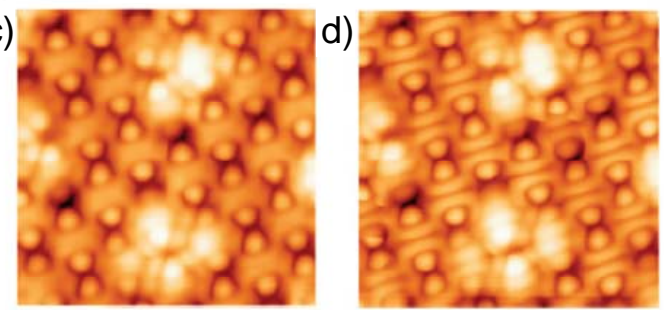

e)

LUMO

f)

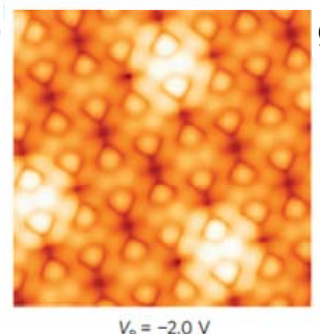

g)

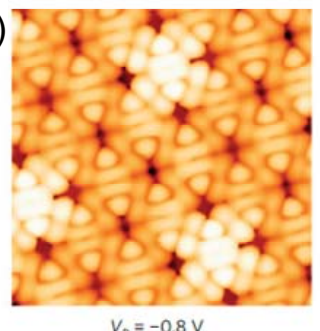

h)
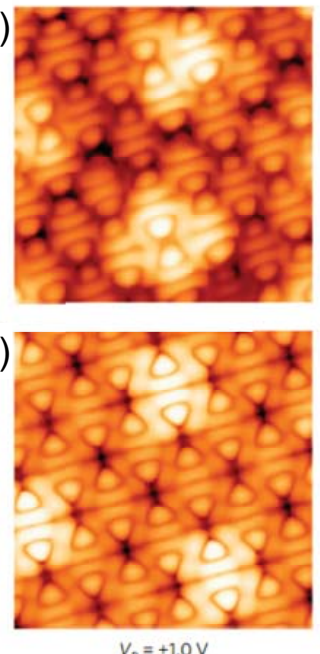

Fig. 24 a) STM image of individual TCNQ molecules adsorbed on graphene $/ \operatorname{Ru}(0001)$ measured at $4.6 \mathrm{~K}$. The white blobs are the upper part of the moiré pattern of graphene/ $\mathrm{Ru}(0001)$ whose unit cell is also indicated. b) Calculated HOMO and LUMO of TCNQ in the gas phase superimposed on the atomic structure. c-e) Spatially extended intermolecular bands in a TCNQ monolayer adsorbed on graphene/ $\mathrm{Ru}(0001)$. Topographic STM images of a TCNQ monolayer on graphene/Ru(0001) recorded at $c) V_{b}=-2 \mathrm{~V}$, d) $V_{b}$ $=-0.8 \mathrm{~V}$ and e) $\mathrm{V}_{\mathrm{b}}=+1 \mathrm{~V}$. f-h) The corresponding simulated topographic images. The bands result from the hybridization of the TCNQ frontier orbitals. Reproduced with permission from ref. 138. Copyright 2013 Nature Publishing Group.

\section{Donor-acceptor interactions of graphene}


The electron energy in graphene is linearly correlated to wave vector near the crossing points in the Brillouin zone. ${ }^{139}$ The charge carrier behaviour of graphene has been described by relativistic particles effect using Dirac equation ${ }^{140}$ which explains the high conductivity and luminescence quenching ability of graphene. ${ }^{141}$ The quenching effect of graphene materials is attributed to three possible mechanisms: i) Förster resonance energy transfer (FRET), ii) surface energy transfer (SET), and iii) photoinduced electron transfer (PET). ${ }^{142-}$ 147 To observe FRET, a suitable pair of energy donor and energy acceptor having appropriate spectral overlap and in proximity $(1-10 \mathrm{~nm})$ are required. ${ }^{148}$ The energy transfer from the excited donor to the acceptor in a non-radiative manner through a dipole-dipole interaction occurs whose transfer efficiency is inversely proportional to the sixth power of the distance between the D-A pair. ${ }^{148}$ In the process of SET, the energy transfer efficiency is inversely proportional to the fourth power of the D-A distance. ${ }^{149,150}$ Consequently, SET is more efficient than FRET at relatively longer distances $(20-30 \mathrm{~nm})$. PET occurs between a luminescent sensitizer (donor or acceptor) and graphene material. ${ }^{142-147}$ The quenching mechanism is a redox reaction between the excited sensitizer and the quencher with electron transferring from the donor to the acceptor. The efficiency of the quenching decreases exponentially as the distance increases.

An important aspect in the graphene research is in the development of functionalized graphene composites with organic/inorganic molecules to obtain improved functional properties. Some of these properties include, i) high electrical conductivity and improved mechanical properties, ii) unique ambipolar electronic property, iii) magnetic features upon hybridization with different magnetic nanoparticles, and iv) intriguing luminescent properties. $^{143-147,151-155}$

\subsection{Ground- and excited-state charge transfer in donor-acceptor, graphene-based supramolecular hybrids}

Interaction of photosensitizers such as Pors, Pcs, etc., with graphene provides electron D-A systems while improving the exfoliation and dispersion stability of the graphene by overcoming the van der Waals interactions between the individual graphene sheets. ${ }^{143-147}$ Electronic interactions both in the ground and excited state could be anticipated in such systems wherein shifting of Fermi energy is expected, thus opening of an electronic gap in graphene. Both non-covalent stacking and covalent functionalization is expected to yield 
such results. Consequently, fundamental studies related to electronic communications and interaction in graphene-based D-A systems are of great interest. In such systems, graphene could act either as an electron donor or acceptor depending on the relative energy levels of the entities. The following section provides summary of these findings.

Matte et al. ${ }^{156}$ reported on the fluorescence quenching by graphene of organic donor molecules such as pyrene butanoic acid succinimidyl ester (PyBS) and oligo( $p$ phenylenevinylene) methyl ester (OPV-ester) (Fig. 25). Absorption and fluorescence spectra of the donor molecules recorded in the mixture with increasing concentrations of graphene showed no change in the former, but remarkable quenching of fluorescence. The property of graphene to quench fluorescence of OPV-ester was shown to be associated with photo-induced electron transfer, on the basis of fluorescence measurements.
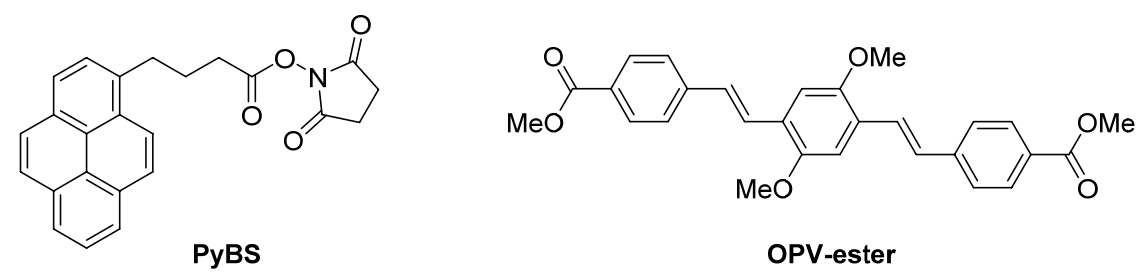

Fig. 25 Molecular structures of pyrene butanoic acid succinimidyl ester (PyBS) and oligo( $p$-phenylenevinylene) methyl ester (OPV-ester).

Supramolecular D-A hybrids composed of few-layer graphene as an electron acceptor and (Pyr) $)_{4} \mathbf{H}_{2} \mathbf{P c}$ or $\left(\mathbf{P y r}_{4}\right) \mathbf{Z n}$ (II)Por bearing four pyrene entities as photosensitizer donors (Fig. 26a) were formulated and characterized by various techniques. ${ }^{157}$ Due to the presence of four pyrene units, strong ground and excited state interactions were observed wherein the fluorescence of both Por and Pc was quenched over $80 \%$ due to interactions with graphene (Fig. 26b,c). Femtosecond transient absorption studies on these hybrids revealed occurrence of ultrafast charge separation whose rates were in the order of $10^{11}-10^{12} \mathrm{~s}^{-1}$. The fast kinetic process was attributed to the close proximity of the donor and acceptor entities in the $\pi$-stacked D-A hybrids. 

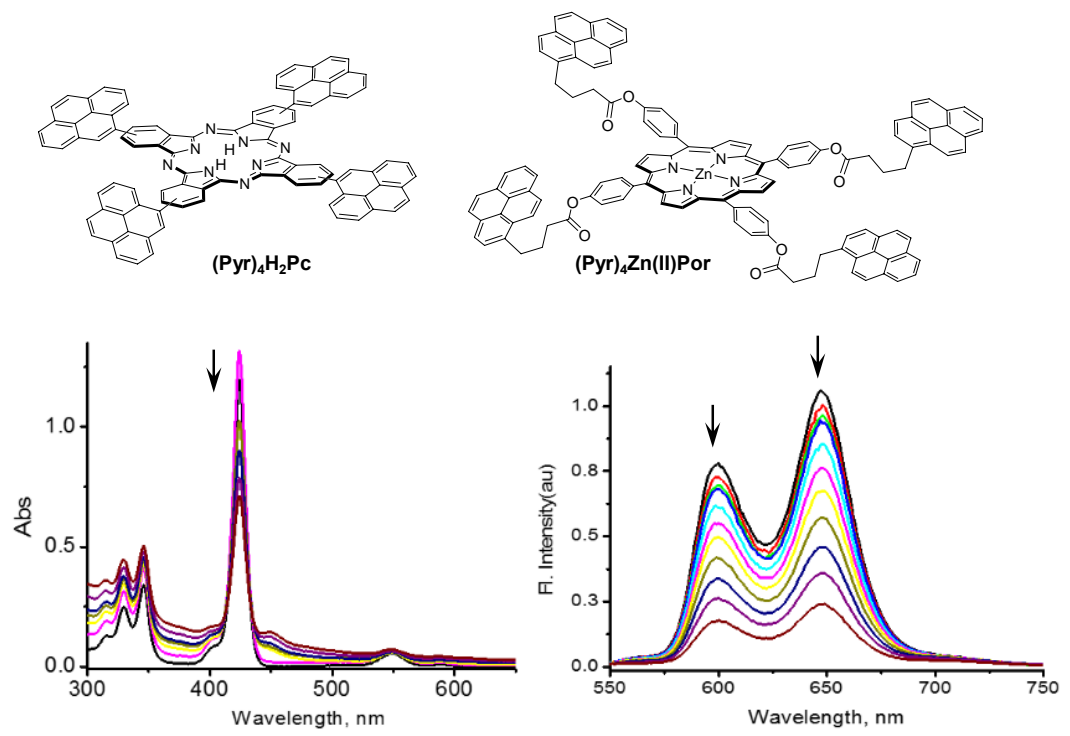

Fig. 26 a) Molecular structure of pyrene-substituted (Pyr) $)_{4} \mathbf{H}_{2} \mathbf{P c}$ and $\left(\mathbf{P y r}_{4}\right) \mathbf{Z n}(\mathrm{II}) \mathbf{P o r}$. b) Optical absorption and c) fluorescence spectral changes of $\left(\mathbf{P y r}_{\mathbf{4}}\right) \mathbf{Z n}(\mathbf{I I}) \mathbf{P o r}$ upon increasing the addition of graphene in $o$-DCB $\left(\lambda_{\mathrm{ex}}=424 \mathrm{~nm}\right)$.

On this connection, D-A nanohybrids were obtained by supramolecular attachment of $n$ type and $p$-type $\mathrm{Zn}(\mathrm{II}) \mathrm{Pc}$-functionalized poly-p-phenylenevinylene oligomers to graphene. These oligomers were able to produce exfoliation of graphite in THF to form stable nanoconjugates which featured charge separation evolving from the photoexcited $\mathrm{Zn}$ (II)Pc to graphene. Prototype solar cells were prepared using these hybrids, although low IPCE values (ca. $1 \%)$ were achieved. ${ }^{158,159}$

Kiessling et al. prepared four novel nanographene/Por hybrids for solar energy conversion schemes. ${ }^{160,161}$ The synthesized Pors differed in their core functionalization (free-base or zinc metallated) or in the number of carboxylic acid groups at the conjugated $\beta$-pyrrolic side chain (one or two). Immobilization of the Pors onto the basal plane of graphene was witnessed by distinct electronic interactions in both the ground and the excited states. In the ground state, a strong loss in oscillator strength accompanied by broadening of the Por transitions provided evidence to the shift of electron density from the electron donating Por to graphene. In Raman spectra, decrease in full width at half maximum of the 2D band was accompanied by an increased intensity ratio of the $2 \mathrm{D}$ band to the $\mathrm{G}$ band. The observed nearly quantitative quenching of the Por fluorescence was indicative of an efficient charge 
transfer. Femtosecond transient absorption measurements corroborated such excited state charge transfer evidenced by the one-electron oxidized radical cation of the Por with absorption maxima at 490 and $625 \mathrm{~nm}$ in the visible region and conduction band electrons in graphene with features at 890 and $1025 \mathrm{~nm}$ in the NIR region. As a proof of concept, solar cells built using these D-A hybrids resulted in the generation of photocurrent and photovoltage.

A double-decker strategy was used to form D-A hybrids by Skaltsas et al. ${ }^{162}$ In this approach, an anionically charged block copolymer poly(isoprene- $b$-acrylic acid) (PI-b$\mathbf{P A A}^{-}$) was utilized to form stable dispersions of exfoliated graphene in aqueous media and then was used to electrostatically bind cationic 5,10,15,20-tetrakis(1-methyl-4pyridinio)Por $\left(\mathbf{H}_{2} \mathbf{P o r}^{4+}\right.$ ) (Fig. 27). ${ }^{162}$ The formed graphene/PI-b-PAA ${ }^{-}-\mathbf{H}_{2} \mathbf{P o r}^{4+}$ ensemble was examined by dynamic light scattering (DLS) and other spectroscopic methods. The steady-state and time-resolved photoluminescence studies were suggestive of electron/energy-transfer phenomena occurring within the nanoensemble. Finally, kinetic analysis of the lifetime profiles of the fluorescence emission gave information regarding the quenching rate constant and quantum yield of the singlet excited state of $\mathbf{H}_{2} \mathbf{P o r}^{4+}$ in the graphene/PI-b-PAA ${ }^{-}-\mathbf{H}_{2}$ Por $^{4+}$ ensemble.

a)
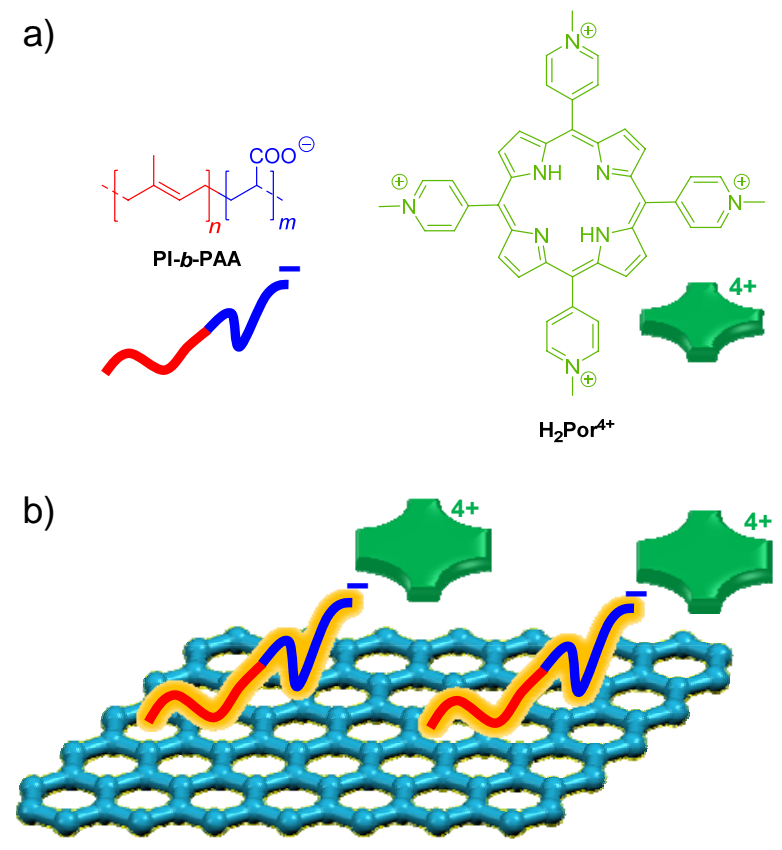
Fig. 27 a) Molecular structure and schematic representation of PI-b-PAA ${ }^{-}$and $\mathbf{H}_{2} \mathbf{P o r}^{4+}$. b) Cartoon of the graphene/PI-b-PAA ${ }^{-}-\mathbf{H}_{2}$ Por $^{4+}$ ensemble, in which the tetracationic $\mathbf{H}_{2}$ Por $^{4+}$ is electrostatically attracted by the negatively charged $\mathbf{P I} \mathbf{-} \boldsymbol{b}-\mathbf{P A} \mathbf{A}^{-}$block copolymer.

In another study, ultrafast electron injection at the cationic Por-graphene interface assisted by molecular flattening was reported by Aly et al. ${ }^{163}$ Three Pors, viz., neutral 5,10,15,20tetra(4-pyridyl)Por, and cationic, meso-substituted 5,10,15,20-tetra(1-methyl-4pyridino)Por, and 5,10,15,20-tetra(4-trimethyl-ammoniophenyl)Por were used to form the hybrid with graphene carboxylate. The negatively charged graphene carboxylate surface caused an external electrostatic constraint exerted on the cationic Pors. Due to this external constraint, the methylpyridinium groups, which initially adopted a nearly perpendicular conformation with respect to the Por's plane, rotates by tens of degrees adopting a coplanar conformation with respect to the macrocycle. The steady-state and femtosecond timeresolved studies demonstrated that the charge transfer process at the Por-graphene carboxylate (GC) interfaces could be switched on and off by changing the electronic nature of the meso sustituents and the metal in the Por cavity.

The studies presented above revealed the role of graphene as an electron acceptor while the organic sensitizers served as electron donor moiety. An example of graphene serving as an electron donor was revealed by assembling it with an alkylsulfonyl $\mathrm{Zn}(\mathrm{II}) \mathrm{Pc}$-pyrene conjugate (Fig. 28). ${ }^{164}$ The presence of the pyrene unit in the $\mathrm{Zn}(\mathrm{II}) \mathrm{Pc}$ conjugate was essential for the non-covalent immobilization of the electron accepting macrocycle onto the basal plane of highly exfoliated graphite. Strong interactions dominated the electronic properties of the nanohybrid both in the ground and excited states. Femtosecond pump probe experiments assisted in corroborating an ultrafast charge separation, that is, the generation of the one-electron reduced radical anion of the $\mathrm{Zn}(\mathrm{II}) \mathrm{Pc}$ and one-electron oxidized graphene after irradiation at $387 \mathrm{~nm}$, followed by slow charge recombination. 


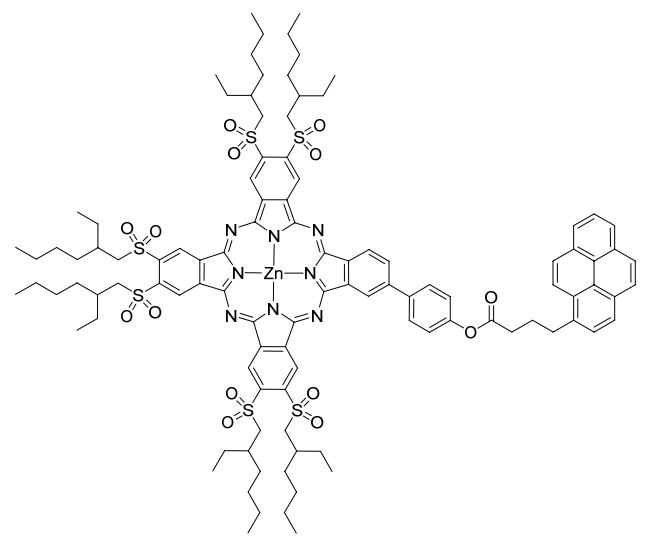

Fig. 28 Molecular structure of pyrene-functionalized, electron-accepting $\mathrm{Zn}$ (II)Pc used to form graphene-based D-A hybrids.

The preparation of exfoliated graphene-Pc nanohybrids, and the investigation of their photophysical properties have been also reported using pyridyl-substituted, electronaccepting Pcs. The functionalized macrocycles in DMF afforded single layer and turbostratic graphene based nanohybrids, which were fully characterized by AFM, TEM, Raman, steady-state and pump probe transient absorption spectroscopy. In this connection, photoinduced charge transfer from graphene to the electron accepting Pcs was found by steady-state and time-resolved spectroscopy experiments. ${ }^{165}$

The possibility to attach multiple Pors to graphene was explored by Economopoulos and Tagmatarchis. ${ }^{166}$ In this approach, firstly, an anionic Por was stabilized onto exfoliated graphene by taking advantage of $\pi-\pi$ interactions, while, at a later stage, a cationic Por was anchored to the former macrocycle through electrostatic interactions (Scheme 6). The same process can be done inverting the Pors' deposition sequence (i.e., using a cationic Por to $\pi$ stack to graphene followed by the addition of an anionic macrocycle). These interactions allowed effective electronic communication of the second Por that was electrostatically attached by quenching its emission. The resulting graphene-Por-Por hybrid was examined using several optical techniques (UV-vis, steady state and time-resolved photoluminescence) while electrochemistry was employed to study the thermodynamically favoured quenching pathways. The ionic macrocycle that was electrostatically stabilized onto the Por-graphene nanoensemble showed lifetimes of an order of magnitude faster than its $\pi-\pi$ stacked analogue suggesting a more efficient quenching pathway. 

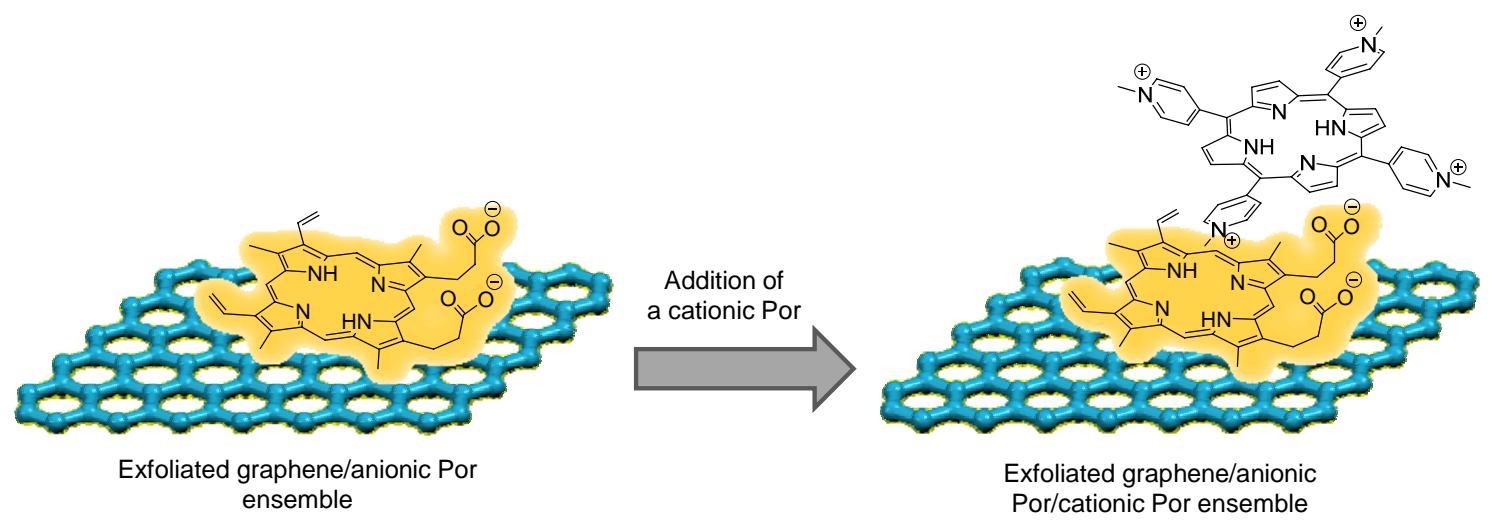

Scheme 6 Schematic representation of the $\pi$-stacking of an anionic Por onto exfoliated graphene followed by the addition of a cationic Por to the as-formed graphene-Por ensemble leading to a graphene-Por-Por multicomponent hybrid.

Apart from Por, Pc and organic dyes, other forms of nanocarbons were also used to form supramolecular D-A hybrids. Ground and excited state interactions in the hybrid system of a film of $\mathrm{C}_{60}$ molecules deposited on single-layer graphene was reported by Heinz and coworkers. ${ }^{167}$ In the absence of photoexcitation, the $\mathrm{C}_{60}$ molecules in the deposited film acted as electron acceptors for graphene, yielding increased hole doping in the graphene layer. Hole doping of the graphene film by a uniform $\mathrm{C}_{60}$ film at a level of $5.6 \times 10^{12} / \mathrm{cm}^{2}$ or 0.04 holes per interfacial $\mathrm{C}_{60}$ molecule was determined by the use of both Raman and terahertz spectroscopy. Further, the transient charge transfer occurring upon photoexcitation by femtosecond laser pulses was also reported. The $\mathrm{C}_{60} /$ graphene hybrid exhibited a short-lived (ps) decrease in terahertz conductivity, followed by a long-lived increase in conductivity. The initial negative photoconductivity transient, which decayed within 2 ps, reflected the intrinsic photo response of graphene. The longer-lived positive conductivity transient, with a lifetime on the order of $100 \mathrm{ps}$, was attributed to photoinduced hole doping of graphene by interfacial charge transfer. This study demonstrated the importance of charge transfer processes at graphene interfaces, both in equilibrium and under photoexcitation, as well as the utility of terahertz spectroscopy in following these processes in real time.

Carbon nanotubes and graphene are among the most widely investigated low-dimensional materials for photonic and optoelectronic devices. ${ }^{168-170}$ It was rationalized that combining 
these two materials into all-carbon hybrid nanostructures would reveal enhanced properties in a range of devices, such as photodetectors and flexible electrodes compared to the individual constituent materials. ${ }^{171}$ Liu et al. ${ }^{172}$ complemented Raman spectroscopy with photocurrent probing, as a robust way of illustrating the interfacial built-in fields, and unambiguously revealing both static and dynamic (photo-induced) charge transfer processes at the nanotube-graphene interfaces. The type of nanotube species used, i.e., metallic as opposed to semiconducting, were also compared. In the examined devices, the graphene sheet was found to be $p$-type doped with $(6,5)$ chirality-enriched semiconducting single-walled carbon nanotubes (SWCNTs), while $n$-type doped with ultra-pure ( $>99 \%$ ) metallic SWCNTs. ${ }^{172}$ This study provided key design guidelines for all-carbon based devices.

A new approach to probe the effect of graphene on photochemical charge separation in D-A conjugates was reported by $\mathrm{KC}$ et al. ${ }^{125}$ For this purpose, multi-modular D-A conjugates comprised of three entities of pyrene, a SubPc and a fullerene, (i.e., (Pyr) $)_{3}$ SubPc- $\mathbf{C}_{60}$ and (PyrPhO) $)_{3} S_{\text {SubPc- }} \mathbf{C}_{60}$ in Fig. 19) were synthesized and studied. These conjugates were hybridized on few-layer graphene via $\pi-\pi$ stacking interactions of the three pyrene entities. These hybrids were characterized using Raman, HR-TEM, spectroscopic and electrochemical techniques. In these conjugates, photoinduced charge separation in the absence and presence of graphene was established from studies involving femtosecond transient absorption spectroscopy. Accelerated charge separation and recombination was observed for the graphene/conjugate hybrids suggesting that these materials could be useful for their incorporation in fast-responding optoelectronic devices, in addition to light energy harvesting applications.

\subsection{Photoinduced charge transfer interactions in donor-acceptor, graphene-based covalent hybrids}

The first report on the covalent attachment of a light-harvesting and electron-donating Pc to the basal plane of few-layer graphene was reported by Ragoussi et al. ${ }^{173}$ Covalent functionalization was achieved in two steps (Scheme 7). First, 1,3-dipolar cycloaddition of the exfoliated graphite in the presence of an excess of $\mathrm{N}$-methylglycine and 4formylbenzoic acid affording carboxyphenyl modified graphene. In the second step, esterification reaction between carboxyphenyl modified graphene and the alcohol- 
terminated $\mathrm{Pc}$ in the presence of $\mathrm{EDC} / \mathrm{HOBt}$ yielding graphene-Pc nanoconjugate. The Pcgraphene hybrid formed stable suspensions in solvents such as NMP and DMF. The Raman and absorption spectral studies were indicative of ground state interactions in the hybrid. Diminished fluorescence quantum yield and lifetime were indicative of excited state interactions in the hybrid. Physicochemical characterization reveals an ultrafast charge separation from the photoexcited Pc to few-layer graphene followed by a slower charge recombination both in DMF and NMP.

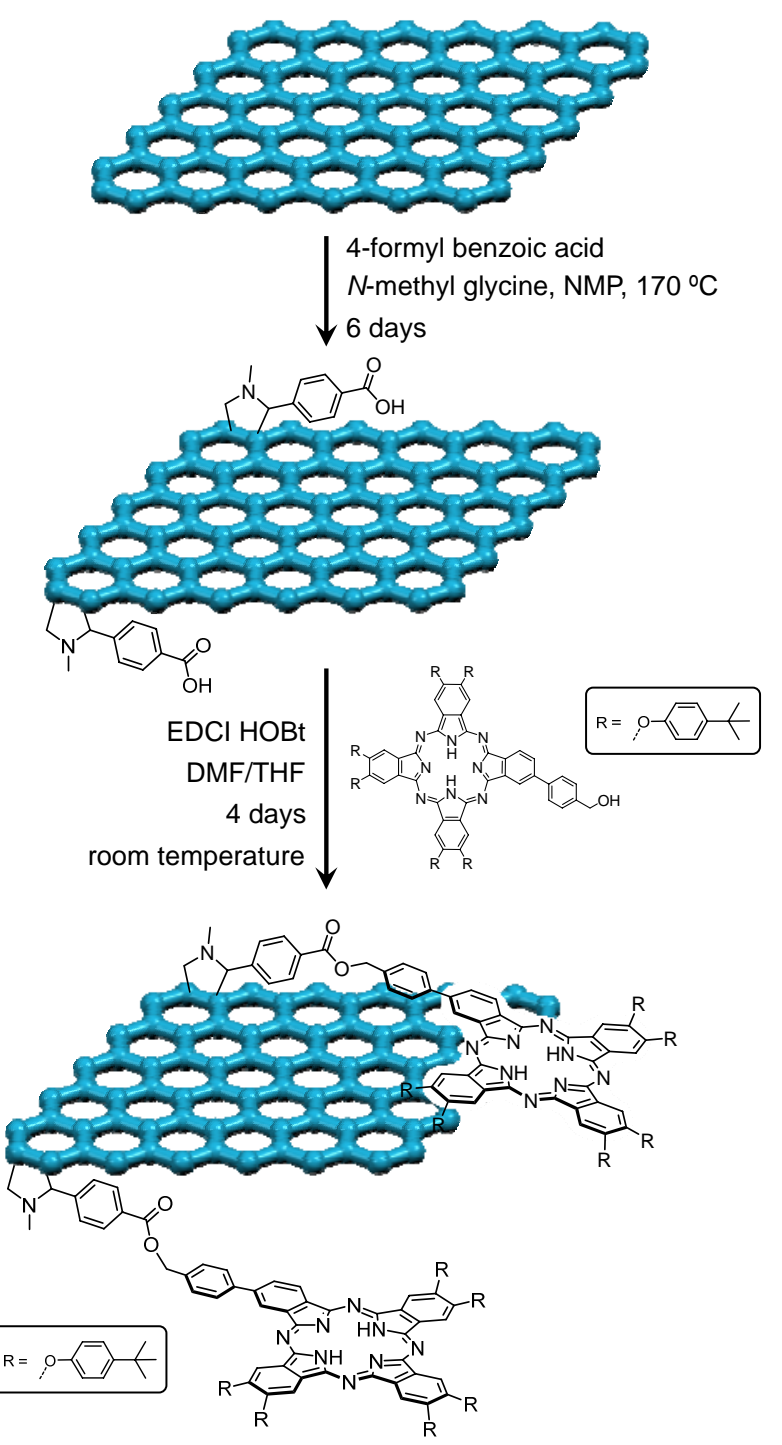

Scheme 7 Synthetic route towards the graphene-Pc nanoconjugate. EDC = 1-ethyl-3-(3dimethylaminopropyl)carbodiimide, $\mathrm{HOBt}=1$-hydroxybenzotriazole. 
A simple procedure for graphene exfoliation upon tip sonication in $o$-DCB was reported by Karousis et al. ${ }^{174}$ This treatment was followed by covalent grafting of (2-aminoethoxy)(tritert-butyl) $\mathrm{Zn}(\mathrm{II}) \mathrm{Pc}$ to exfoliated graphene sheets (Scheme 8). The Zn(II)Pc-graphene hybrid material was found to be soluble in common organic solvents without any precipitation for several weeks. Initial characterization of the $\mathrm{Zn}(\mathrm{II}) \mathrm{Pc}-$ graphene hybrid was carried out using diverse spectroscopic techniques, while TGA allowed to determine the amount of $\mathrm{Zn}(\mathrm{II}) \mathrm{Pc}$ loaded onto graphene. Efficient fluorescence quenching of $\mathrm{Zn}$ (II)Pc in the $\mathrm{Zn}(\mathrm{II}) \mathrm{Pc}$-graphene hybrid material suggested occurrence of photoinduced events from the photoexcited $\mathrm{Zn}(\mathrm{II}) \mathrm{Pc}$ to graphene. The dynamics of the PET was evaluated by femtosecond transient absorption spectroscopy revealing the formation of transient species such as $\mathrm{Zn}(\mathrm{II}) \mathrm{Pc}^{{ }^{+}}$, yielding the charge-separated state $\mathrm{Zn}(\mathrm{II}) \mathrm{Pc}^{{ }^{+}-\text {graphene }}{ }^{--}$. Finally, the $\mathrm{Zn}(\mathrm{II}) \mathrm{Pc}$-graphene hybrid material was integrated into a photoactive electrode of an optical transparent electrode (OTE) cast with nanostructured $\mathrm{SnO}_{2}$ films $\left(\mathrm{OTE} / \mathrm{SnO}_{2}\right)$, which exhibited stable and reproducible photocurrent responses, and decent IPCE.

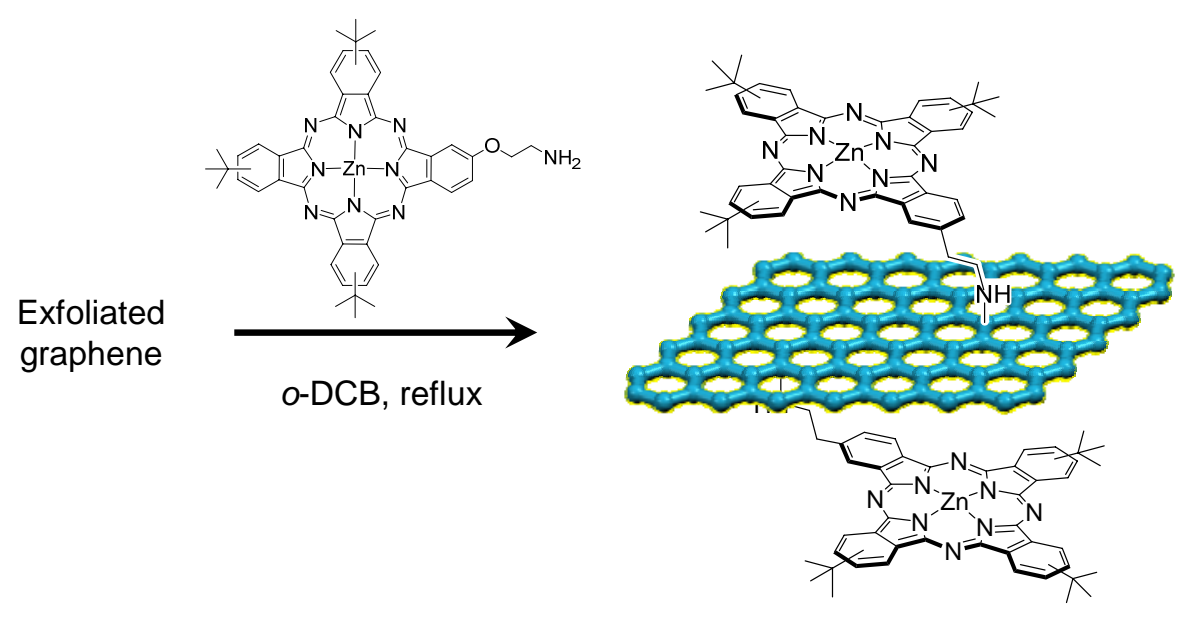

Scheme 8 Covalent functionalization of exfoliated graphene sheets with $\mathrm{Zn}$ (II)Pc.

The first example of highly exfoliated graphene (EG) covalently linked to electron accepting Pcs was reported by the groups of Torres and Guldi. ${ }^{175}$ In that work, the functionalization of the nanocarbon surface was accomplished using alkylsulfonyl Zn(II)Pcs by means of alkyne-azide "click" chemistry (Scheme 9). Upon complete characterization of the resulting graphene-Pc hybrid using various physicochemical techniques, electron D-A interactions both in the ground and in the excited state were 
probed revealing PET from graphene to the electron-accepting $\mathrm{Zn}$ (II)Pc. This example served graphene as an electron donor in contrast to the previous examples where graphene was largely behaving as electron acceptor.

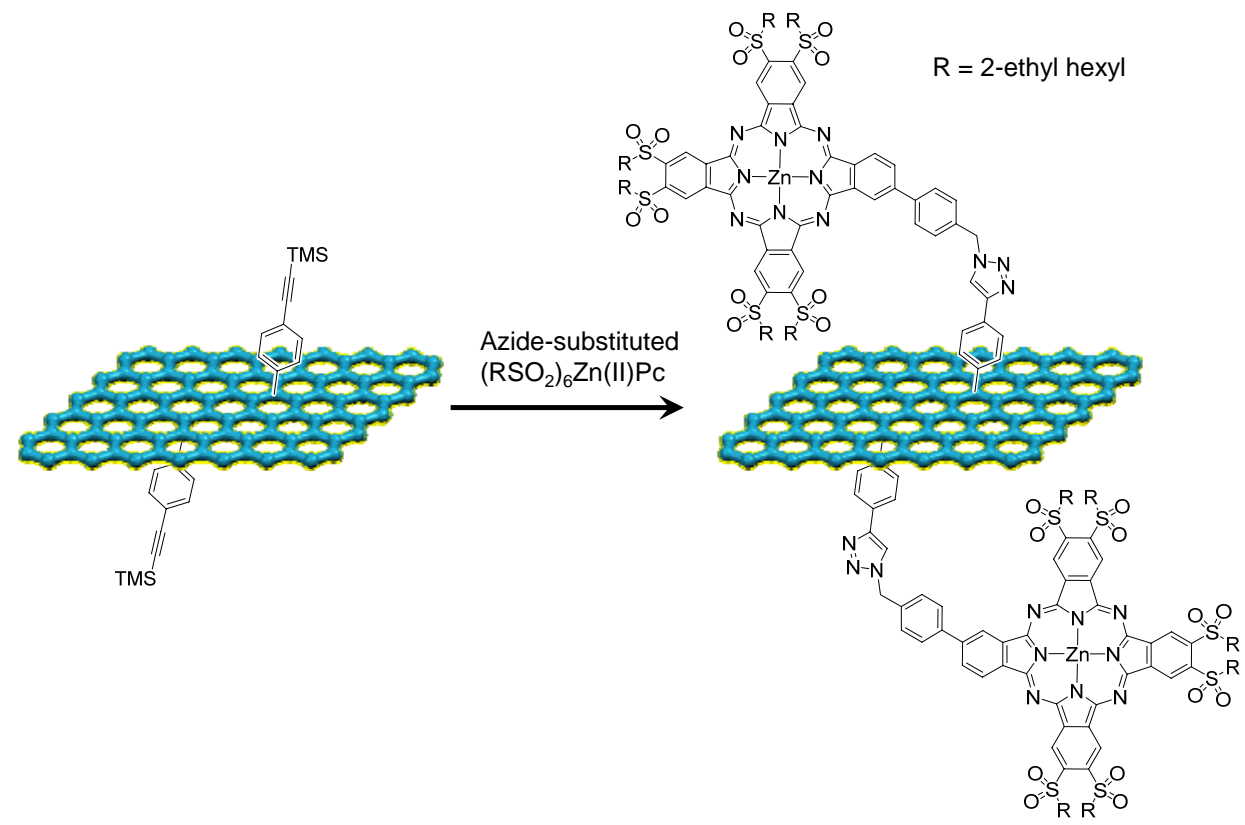

Scheme 9 Covalent grafting of electron accepting $\mathrm{Zn}(\mathrm{II}) \mathrm{Pc}$ on exfoliated graphene by means of alkyne-azide "click" chemistry (see Ref. 175 for experimental details).

\subsection{Photoinduced charge transfer interactions in donor-acceptor, graphene oxide- and reduced graphene oxide-based supramolecular hybrids}

Functionalized GO and $\mathrm{rGO}$ have received much attention in a broad range of applications from light harvesting devices to biological sensors. ${ }^{176,177}$ The following section highlights some of the key findings in the preparation and study of D-A supramolecular ensembles between electroactive molecules and graphene oxide- and reduced graphene oxide.

The ability of chemically rGO to act as an energy and electron transfer agent was first probed by Wojcik and Kamat. ${ }^{178}$ The authors used a cationic Por, viz., 5,10,15,20tetrakis(1-methyl-4-pyridinio)Por (TMPyPor) to demonstrated charge-transfer interactions with chemically rGO. Formation of the ground-state TMPyPor-rGO complex in solution was marked by the red-shift of the Por absorption band Whereas the Por fluorescence lifetime diminished from 5 to 1 ns upon complexation with rGO, indicating excited-state interaction between singlet excited Por and rGO. Femtosecond transient absorption 
measurements carried out with TMPyPor adsorbed on rGO film revealed fast decay of the singlet excited state, followed by the formation of a longer-living product with an absorption maximum around $515 \mathrm{~nm}$ indicating the formation of a Por radical cation. The ability of TMPyPor-rGO to undergo photoinduced charge separation was further confirmed from the photoelectrochemical measurements. TMPyP-rGO coated conducting glass electrodes were capable of generating photocurrent under visible excitation. These results were indicative of the electron transfer between photoexcited Por and rGO. Further, the role of graphene in accepting and shuttling electrons in light-harvesting assemblies was discussed.

Photoinduced charge separation in ordered self-assemblies of $N, N^{\prime}$-di(2(trimethylammonium iodide) ethylene) perylenediimide (TAI-PDI)-GO hybrid layers in water was investigated by Supur et al. ${ }^{179}$ PDI served as an ideal building block for nanohybrids of GO because its strong absorption in the visible region and the low reduction potential making it a powerful light harvester and a good electron acceptor. The cationic groups on TAI-PDI enhanced the electrostatic interactions with the functional groups of GO, such as carboxylic acids, and its large aromatic plane established strong $\pi-\pi$ interactions with the $\pi$-surface of the GO sheet. PET dynamics of self-assemblies of TAIPDI-GO hybrids were investigated by using femtosecond laser-induced transient absorption spectroscopy. The femtosecond transient spectra of aggregates of (TAI-PDI)GO in water displayed a very fast formation of broad transient absorption at around 720 and $960 \mathrm{~nm}$ after the selective excitation of PDI at $510 \mathrm{~nm}$. These transient features agreed well with those of the radical anion of PDI providing a valid proof for PET from GO to ${ }^{1} \mathrm{PDI} *$. The rate of charge separation $\left(k_{\mathrm{CS}}\right)$ was estimated to be $3.6 \times 10^{11} \mathrm{~s}^{-1}(\tau=2.8 \mathrm{ps})$. The fast formation rate suggested that the charge separation was very efficient ruling out the other quenching mechanisms such as energy transfer. From the bi-exponential decay, charge recombination of $31 \mathrm{ps}\left(k_{\mathrm{CR} 1}=3.2 \times 10^{10} \mathrm{~s}^{-1}\right)$ and $417 \mathrm{ps}\left(k_{\mathrm{CR} 2}=2.4 \times 10^{9} \mathrm{~s}^{-1}\right)$ were arrived. The relatively slow decay was accounted for the presence of the electron migration mechanism among the $\pi$-stack of PDI during a possible PET process with the GO sheet. Using a layer-by-layer self-assembly method, rGO-based composite films with high photoelectronic activity were assembled with 5,10,15,20-tetrakis $(p-N, N, N$ trimethylanilinium)Por tetraiodide (TAPPI) and tetrasulfonated $\mathrm{Cu}(\mathrm{II}) \mathrm{Pc}$ as the co- 
sensitizers by Zhang et al. ${ }^{180}$ The $\pi-\pi$ and electrostatic interactions were the main driving forces of the $\mathrm{rGO} / \mathrm{TAPPI} / \mathrm{Cu}$ (II)Pc composite film assembly. The linear dependence of the absorption on the layer numbers of the films demonstrated the formation of the ordered films. In the composite film, an efficient PET was evidenced by fluorescence spectra. Furthermore, the photoelectronic response for the $\mathrm{rGO} / \mathrm{TAPPI} / \mathrm{Cu}(\mathrm{II}) \mathrm{Pc}$ film was higher than that of the $\mathrm{rGO} / \mathrm{TAPPI}$, the $\mathrm{rGO} / \mathrm{Cu}(\mathrm{II}) \mathrm{Pc}$ or the TAPPI/Cu(II)Pc films. The complementary absorption spectra of TAPPI and $\mathrm{Cu}(\mathrm{II}) \mathrm{Pc}$ and the quick transfer of photoproduced electrons were reasoned as the main cause for the enhanced photoelectronic response in the $\mathrm{rGO} / \mathrm{TAPPI} / \mathrm{Cu}(\mathrm{II}) \mathrm{Pc}$ film.

The group of Fukuzumi reported on 3-dimensional (3-D) ordered assemblies of GO layers functionalized with tetrakis(1-methylpyridinium-4-yl)Por $p$-toluenesulfonate, $N, N^{\prime}$-di(2(trimethylammonium iodide)ethylene) perylenediimide (PDI), and $\mathrm{Zn}(\mathrm{II}) \mathrm{Pc}$ tetrasulfonic acid in water. ${ }^{181}$ The molar ratio between the cationic dyes (i.e., Por and PDI) was found to be essential. These cationic chromophores act as a "glue" to combine the GO layers and the anionic $\mathrm{Zn}(\mathrm{II}) \mathrm{Pc}$, helping dispersing the GO layers. The resulting 3-D structures were capable of harvesting light from the ultraviolet to the NIR region. Dye molecules were arranged in mainly lateral order on the GO layers with partial stacking, which allowed direct interactions with the $\pi$-conjugations of the GO surface in 3-D architecture. Ultrafast charge separation upon the photoexcitation of the dyes in the visible/NIR region was observed in these assemblies, in which $\mathrm{Zn}$ (II)Pc and PDI were the ultimate electron donor and acceptor, respectively. Lateral charge migration among the partially stacked dye molecules was inferred from the decay characteristics of the radical ion pair. Triggered by the charge separation processes in the 3-D ordered self-assemblies, significantly higher photocurrent density in the OTE/ $\mathrm{SnO}_{2}$ electrode deposited with self-assemblies of (GOPor-PDI-Zn(II)Pc $)_{n}$ was generated compared to those deposited with only GO or dye components.

\subsection{Photoinduced charge transfer interactions in donor-acceptor, graphene oxide- and reduced graphene oxide-based covalent hybrids}

A successful covalent functionalization of GO with 5-(4-aminophenyl)-10,15,20-triphenyl21,23-Por $\left(\mathrm{H}_{2}\right.$ Por) has been reported by Karousis et al. ${ }^{182}$ The synthetic procedure is outlined in Scheme 10. The resulting $\mathrm{GO}-\mathrm{H}_{2}$ Por hybrid material formed stable dispersions 
in DMF and was characterized by spectroscopic (UV-vis, ATR-IR, Raman) and thermal (TGA) tools. Microscopy techniques (AFM and TEM) were employed to probe the morphological characteristics as well as to investigate the exfoliation of graphene sheets. Steady-state and time-resolved fluorescence emission studies revealed efficient fluorescence quenching, suggesting that electron transfer occurs from the singlet excited state of the $\mathrm{H}_{2}$ Por moiety to the GO sheet. The electrochemical redox potentials of the graphene- $\mathrm{H}_{2}$ Por material were studied by cyclic voltammetry (CV) and differential pulse voltammetry (DPV), and the energy gap for the charge-separated state of (GO) ${ }^{\cdot-}-\left(\mathrm{H}_{2} \mathrm{Por}\right)^{\cdot+}$ was calculated as $0.87 \mathrm{eV}$, while, the negative free-energy change for the photoinduced charge-separation of $\mathrm{GO}-\mathrm{H}_{2}$ Por was evaluated to be $-1.00 \mathrm{eV}$, which confirmed the thermodynamically favourable formation of $(\mathrm{GO})^{--}-\left(\mathrm{H}_{2} \mathrm{Por}\right)^{\cdot+}$. Photoexcitation resulted in the one-electron oxidation of the $\mathrm{H}_{2}$ Por and the simultaneous one-electron reduction of GO, yielding $(\mathrm{GO})^{\cdot-}-\left(\mathrm{H}_{2} \mathrm{Por}\right)^{\cdot+}$, as revealed by transient absorption measurements. The GO$\mathrm{H}_{2}$ Por hybrid material was subsequently deposited by electrophoretic deposition onto $\mathrm{SnO}_{2}$ electrodes, and the $\mathrm{OTE} / \mathrm{SnO}_{2} / \mathrm{GO}-\mathrm{H}_{2}$ Por electrode exhibited a moderate IPCE of $1.3 \%$ in a standard photoelectrochemical cell.

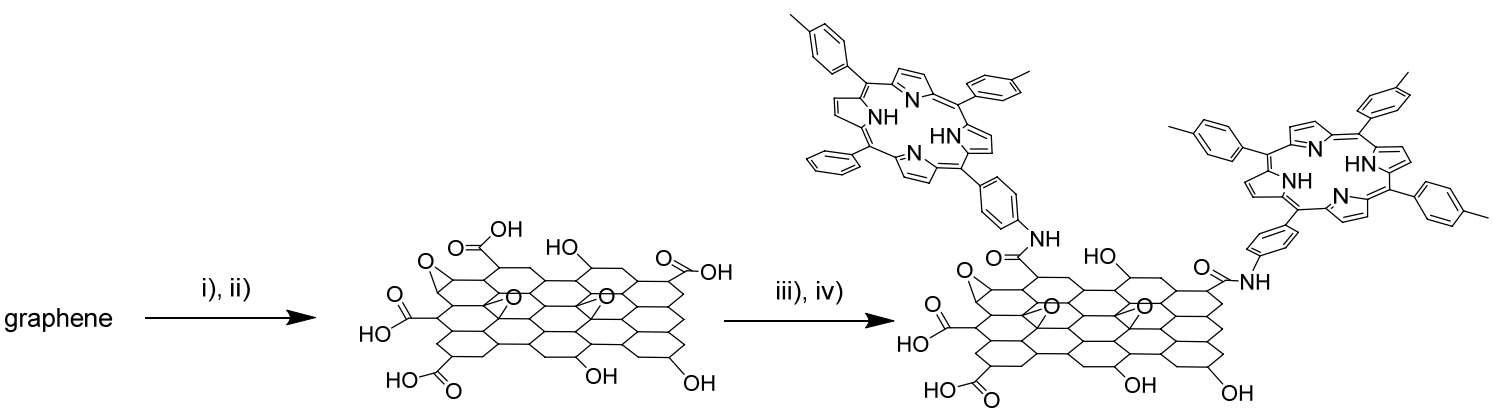

Scheme 10 Synthesis of covalently-linked $\mathrm{H}_{2}$ Por-GO hybrids. i) $\mathrm{H}_{2} \mathrm{SO}_{4} / \mathrm{HNO}_{3}(2: 1 \mathrm{v} / \mathrm{v})$; ii) $\mathrm{KClO}_{3}, 96 \mathrm{~h}$; iii) $\mathrm{COCl}_{2}, 80{ }^{\circ} \mathrm{C}, 24 \mathrm{~h}$; iv) 5-(4-aminophenyl)-10,15,20-triphenyl-21,23Por, THF, room temperature, $72 \mathrm{~h}$.

Using another synthetic methodology, chemically converted graphene (CCG) covalently linked with Pors was prepared by a Suzuki coupling reaction between iodophenylfunctionalized CCG and Zn(II)Por boronic ester as shown in Scheme $11{ }^{183}$ In this case, the covalently linked $\mathrm{CCG}-\mathrm{Zn}(\mathrm{II})$ Por composite was designed to possess a short, rigid phenylene spacer between the $\mathrm{Zn}(\mathrm{II})$ Por and the CCG. The composite material formed 
stable dispersions in DMF and the structure was characterized by spectroscopic, thermal, and microscopic measurements. In steady-state photoluminescence spectra, the emission from the Por linked to the CCG was quenched strongly relative to that of the Por reference. In contrast to the previous report (covalently-linked $\mathrm{H}_{2}$ Por-GO hybrid in Scheme 10), fluorescence lifetime and femtosecond transient absorption measurements of the Por-linked CCG revealed a short-lived Zn(II)Por singlet excited state (38 ps) without yielding the Por radical cation. This observation substantiated the occurrence of energy transfer from the $\mathrm{Zn}(\mathrm{II})$ Por excited state to the CCG and subsequent rapid decay of the CCG excited state to the ground state. Accordingly, the photocurrent action spectrum of a photoelectrochemical device with a $\mathrm{SnO}_{2}$ electrode coated with the Por-linked CCG exhibited no photocurrent response from the Por absorption.

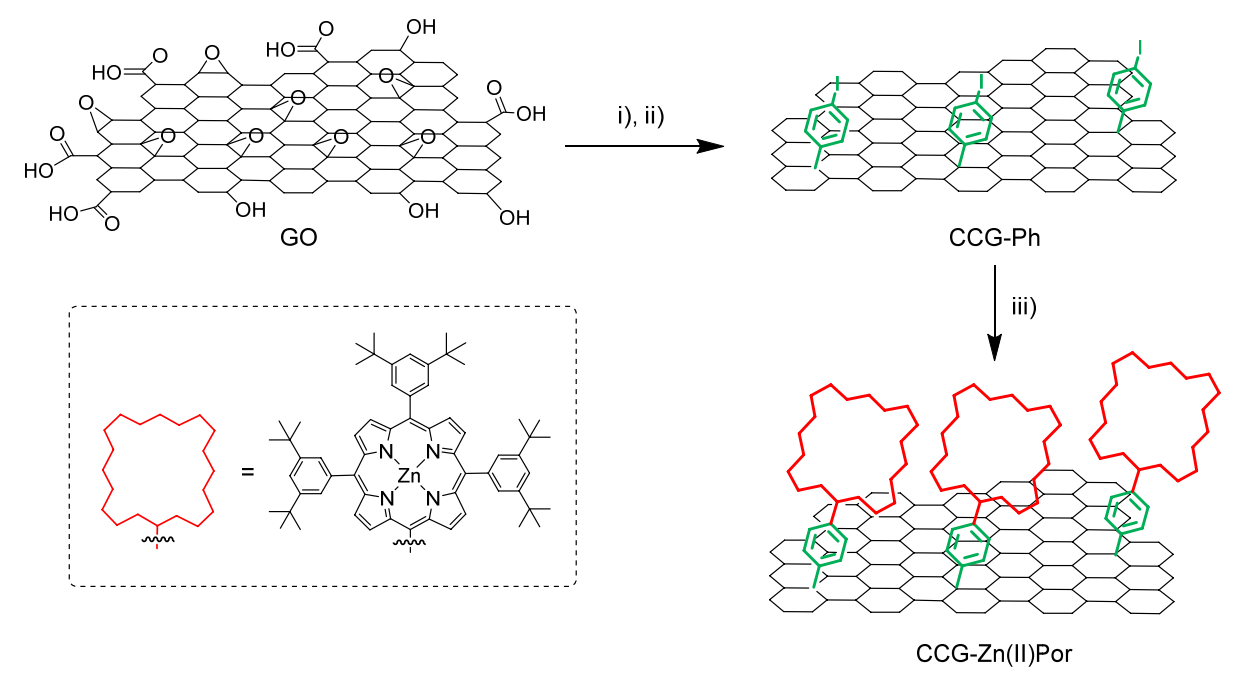

Scheme 11 Synthesis of Por-linked CCG. i) Sodium dodecylsulphate (SDS), $\mathrm{N}_{2} \mathrm{H}_{4} \cdot \mathrm{H}_{2} \mathrm{O}$, $\mathrm{NH}_{3}$ (aqueous), $95^{\circ} \mathrm{C}, 1 \mathrm{~h}$; ii) 4-iodobenzenediazonium tetrafluoroborate, $\mathrm{H}_{2} \mathrm{O}$, room temperature, $\quad 2 \mathrm{~h}$; iii) 5,10,15-tris(3,5-di-tert-butylphenyl)-20-(4,4,5,5tetramethyl[1,3,2]dioxaborolan-2-yl)Zn(II)Por, $\left[\mathrm{Pd}\left(\mathrm{PPh}_{3}\right)_{4}\right], \mathrm{Cs}_{2} \mathrm{CO}_{3}, \mathrm{DMF}, 90^{\circ} \mathrm{C}, 24 \mathrm{~h}$.

Kalita et al. ${ }^{184}$ utilized a redox active Fc instead of previously mentioned Por-based sensitizers to covalently decorate GO (Fig. 29). The insulating GO was partially reduced to improve the conductivity and modified with the Fc molecules. TEM, elemental mapping, $\mathrm{X}$-ray photoelectron and UV-vis spectroscopy studies confirm that the Fc molecules were 
grafted to the surface of a GO sheet. Photoresponsivity of the prepared GO-Fc composite was investigated by fabricating a metal/GO-Fc/metal device. The fabricated device showed enhanced current density under light illumination, suggesting a photo-induced charge transfer process in the developed GO-Fc composite.

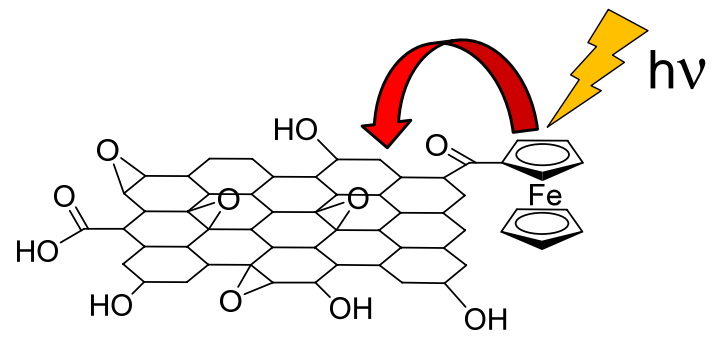

Fig. 29 Schematic representation of the GO-Fc hybrid and photoinduced charge transfer process.

The electron insulating property of GO along with the presence of several organic functional groups on its surface prompted Das et al. to use this material as a platform to anchor donor and acceptor entities. ${ }^{185}$ In this context, single layer graphene oxide (SLGO) was decorated with $\mathrm{C}_{60}$ fullerene as an electron acceptor and a photo-sensitizer $\mathrm{Zn}$ (II)Pc as an electron donor (Zn(II)Pc-SLGO-C $\mathbf{C}_{60}$ in Fig. 30a). The D-A functionalized SLGO was characterized using various techniques including UV-vis, fluorescence, TEM, Raman, TGA and electrochemistry. Photoinduced charge separation leading to $\mathrm{Zn}(\mathrm{II}) \mathrm{Pc}^{\circ+}-\mathrm{SLGO}-\mathrm{C}_{60}{ }^{-}$ charge separated state with a lifetime in the range of $0.04 \mu$ s was established from nanosecond transient studies. In a subsequent study, these D-A decorated hybrids were subjected to a photocatalytic electron pooling experiment. ${ }^{186}$ Electron accumulation in the form of one-electron reduced product of methyl viologen (MV) was obtained in high yields

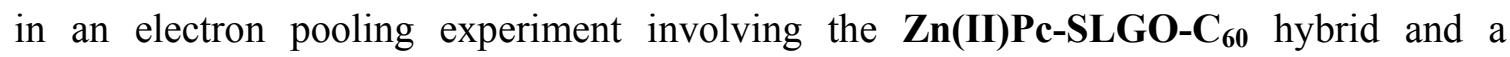
sacrificial electron donor compared with control hybrids involving either Zn(II)Pc-SLGO or $\mathbf{C}_{60}$-SLGO hybrids (see Fig. 30b,c). This novel property of Zn(II)Pc-SLGO-C 60 hybrid has been ascribed to the proximity effect offered by GO with covalently linked donor and

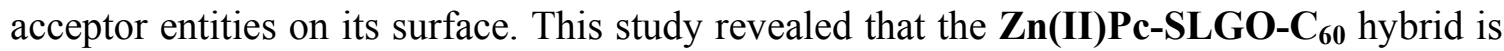
a suitable catalyst for solar fuel production. 
a)
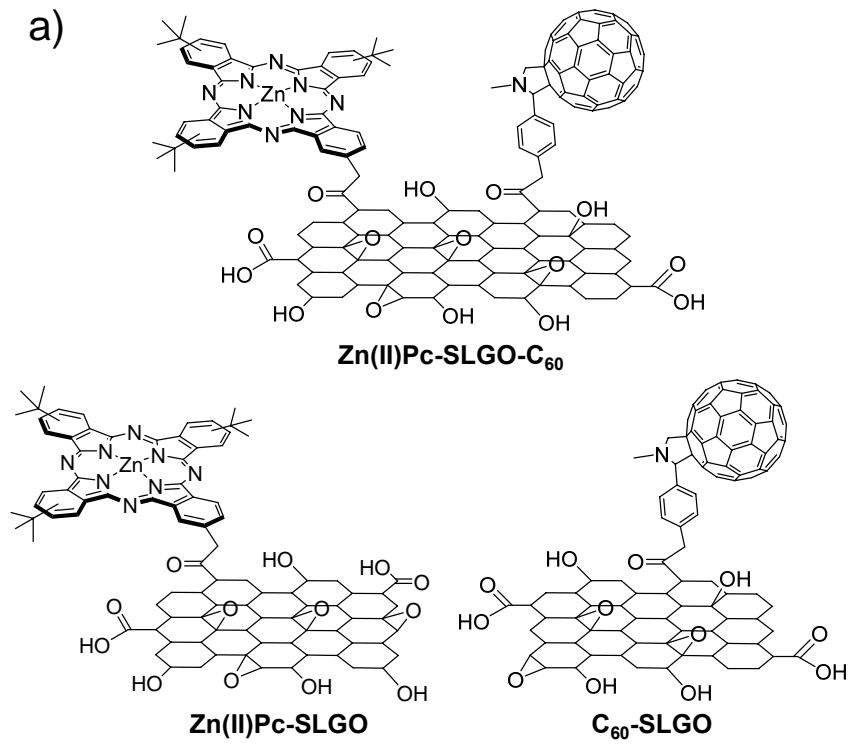

b)
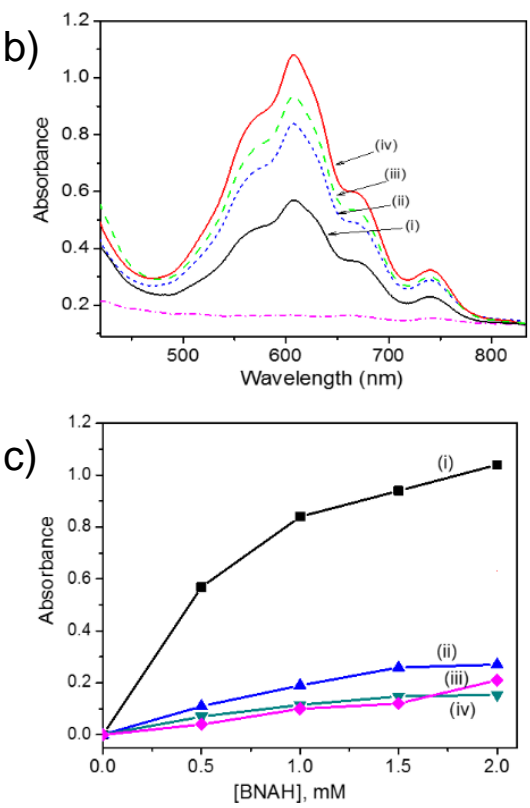

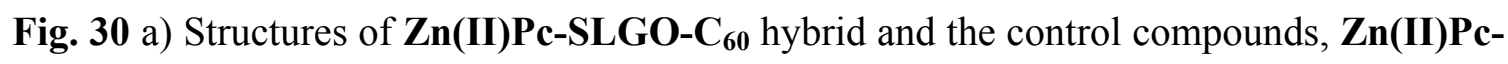

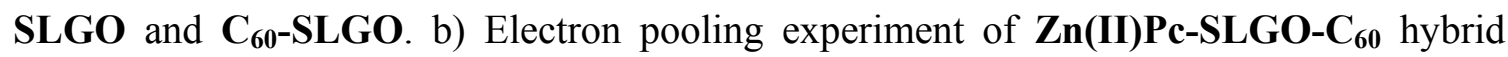
dissolved in $0.5 \mathrm{mM} \mathrm{MV}^{2+}$ in DMF i) in absence of BNAH, and ii-v) upon the addition of increasing amounts of BNAH. c) Extent of $\mathrm{MV}^{\bullet-}$ formation for i) $\mathbf{Z n}(\mathbf{I I}) \mathbf{P c - S L G O - \mathbf { C } _ { 6 0 }}$ hybrid, ii) $\mathrm{C}_{60}$ fulleropyrrolidine, iii) $\mathbf{Z n ( I I ) P c - S L G O}$ hybrid, and iv) mixture of $\mathrm{MV}^{2+}$ and BNAH with no sensitizers.

The synthesis of a pyrene tethered GO nanosheet (Pyr-GON, Fig. 31) was reported by Kim et $a l .{ }^{187}$ An $n$-hexyl and an $n$-butyl spacer were introduced to provide an accurate distance between GON and pyrene molecule. A mechanistic study of the interfacial fluorescence quenching and its recovery processes between a pyrene derivative, namely, 1(aminohexyl)-1-pyrenebutyricamide (Pyr), and nanometer-sized GO sheets was performed. Owing to strong $\pi-\pi$ interactions with Pyr, GON quenched the photoluminescence from pyrene in "folded" conformation of Pyr-GON. On the other hand, the treatment of PyrGON with sodium dodecyl sulphate (SDS) helped Pyr overcome this strong pyrene/GON interactions and causes Pyr-GON to adopt an "unfolded" conformation in which the fluorescence of pyrene was restored. By time-resolved spectroscopy, the ultrafast energy and charge transfer behaviour ( $<1$ ps) was observed especially in "folded" conformation. In addition, photocurrent analysis under white light illumination lead the authors to 
distinguish electron transfer behaviour in "folded" and "unfolded" conformation; wherein charge separation occurred only in "folded" conformations. Overall, these findings provided fundamental understanding of the photophysics of pyrene-functionalized GON nanostructures (Pyr-GON) which could be utilized in nanosheet-electrode based sensors and light harvesting devices.

a)

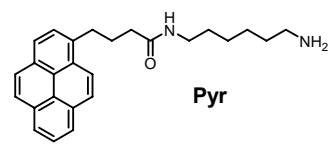

b)

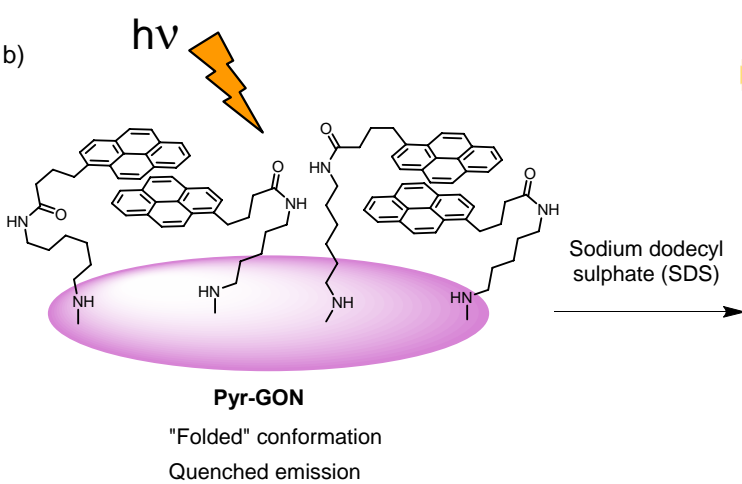

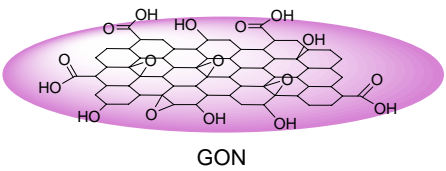
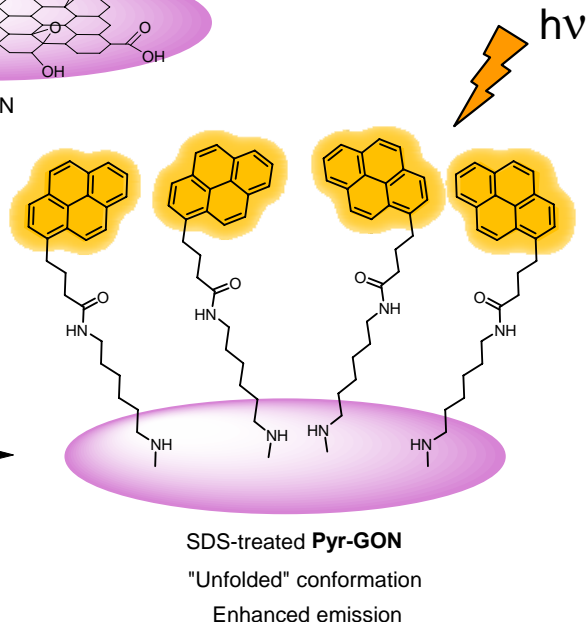

Fig. 31 a) Molecular structure of pyrene-substituted derivative (Pyr) used to functionalize the GON, schematically represented as a violet oval, and b) folded Pyr-GON and unfolded SDS-treated Pyr-GON composites.

In a recent study, ${ }^{188}$ tetracarboxylic $\mathrm{Zn}(\mathrm{II}) \mathrm{Pc}$-amino functionalized GO (Zn(II)PcC $\mathbf{P}_{4}$ NGO) hybrid materials were prepared by a covalent functionalization method (Scheme 12). The characterizations indicated that the amino-functionalization of GO had an important influence on the structure and photophysical properties of the $\mathbf{Z n ( I I ) P c C} \mathbf{P C}_{4}-\mathbf{N G O}$ hybrid. The $\mathbf{Z n}(\mathbf{I I}) \mathbf{P c C}_{4}-\mathbf{N G O}$ hybrid exhibited enhanced PET/energy transfer, compared to the $\mathrm{Zn}(\mathrm{II}) \mathrm{PcC}_{4}$ covalent functionalized $\mathrm{GO}\left(\mathbf{Z n}(\mathbf{I I}) \mathbf{P c C}_{4}-\mathbf{G O}\right)$, owing to the presence of the extended $\mathrm{sp}^{2}$ carbon configurations, along with the partial reduction of the NGO nanosheets and the introduction of electron-donating ethylenediamine. The nonlinear optical (NLO) properties of the hybrids were investigated using the Z-scan technique at $532 \mathrm{~nm}$ with $4 \mathrm{~ns}$ laser pulses. The results showed efficient covalent functionalization and partial reduction of NGO causing the $\mathbf{Z n ( I I ) P c C} \mathbf{P}_{4}-\mathbf{N G O}$ hybrid to possess larger NLO properties than the individual $\mathrm{NGO}, \mathrm{Zn}(\mathrm{II}) \mathrm{PcC}_{4}$ and the $\mathbf{Z n}(\mathbf{I I}) \mathbf{P c C}_{4}-\mathbf{G O}$ hybrid. The enhanced NLO 
performance was attributed to the increased excited state absorption from the extended $\mathrm{sp}^{2}$ carbon configurations of the NGO moiety, reverse saturable absorption arising from $\mathrm{Zn}(\mathrm{II}) \mathrm{PcC}_{4}$ moiety, and the contribution of the efficient PET/energy transfer process between the $\mathrm{Zn}(\mathrm{II}) \mathrm{PcC}_{4}$ and $\mathrm{NGO}$ moieties in the hybrid.

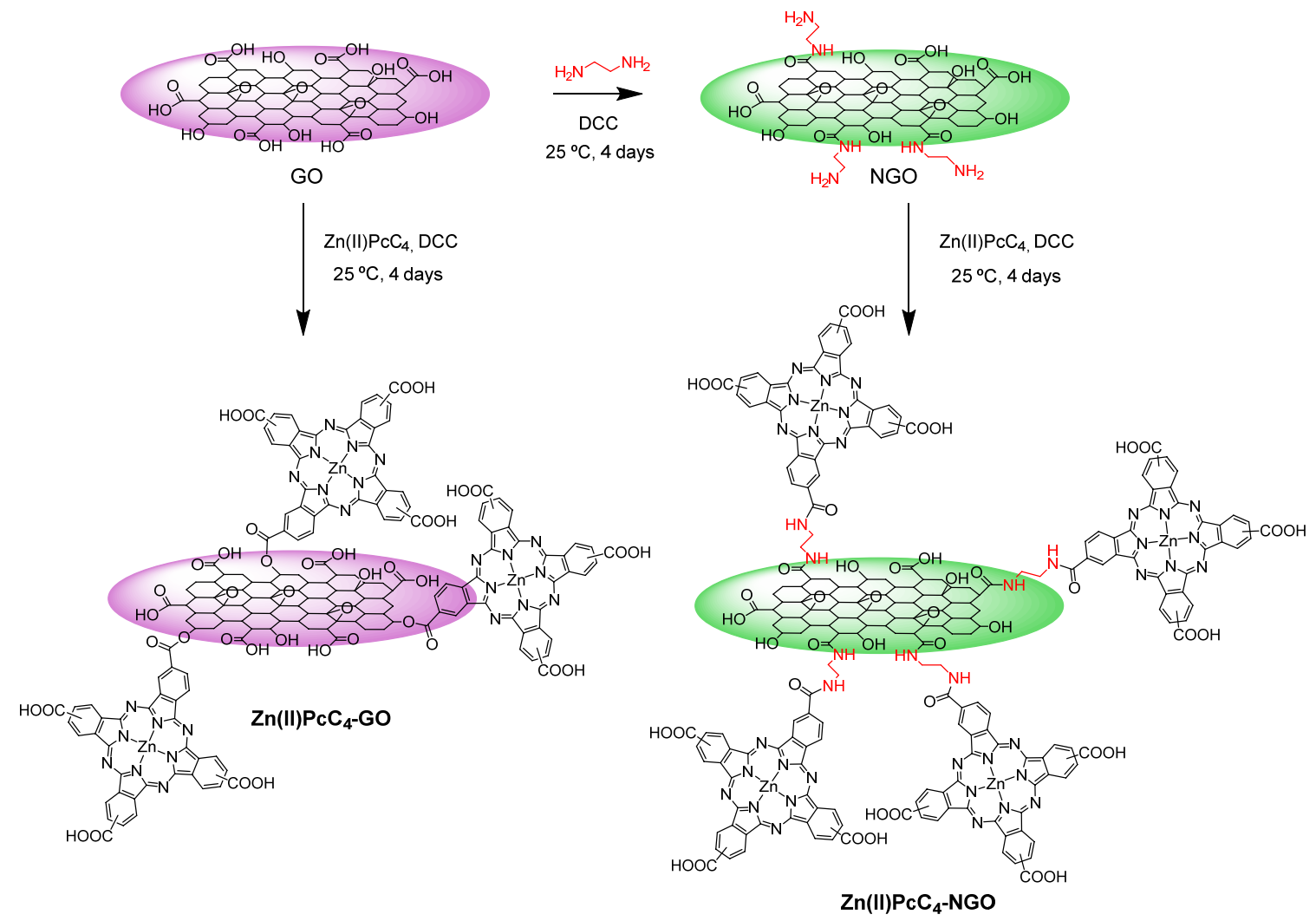

Scheme 12 Synthetic procedure for the preparation of $\mathbf{Z n}(\mathbf{I I}) \mathbf{P c C}_{4}-\mathbf{G O}$ and $\mathbf{Z n}(\mathrm{II}) \mathbf{P c C}_{4-}$ NGO hybrids.

In summary, the D-A systems built via non-covalent and covalent procedures with monolayer and few-layer graphene have revealed strong interactions between the entities. From their spectral characteristics, the origin of these interactions could be attributed primarily to charge transfer. Moderate level of covalent functionalization retained the $\pi$-structure of graphene thus making it useful for optoelectronic applications. D-A type interactions in GO and rGO materials have also been observed both in the supramolecularly assembled and covalently linked systems. Photophysical studies, using femtosecond transient spectral technique and time-resolved emission, have revealed ultrafast phenomenon, mainly due to the close association of the sensitizer-graphene entities. Clever design of graphene 
molecular designs have started emerging out, these include tuning the properties of the sensitizer molecule to make graphene an electron donor or an electron acceptor. Alternatively, D-A decorated graphene systems have shown to be capable of tuning the kinetics of photophysical properties. Much could be expected in the coming years from this fascinating research area.

\section{Conclusions and Outlook}

Since its discovery more than a decade ago, graphene, a physics Nobel prize-worth material, has drawn more attention of many scientists working in fields such as chemistry, physics, or materials science, than any other material before. Although, arguably, graphene is still considered the "golden boy" among GBMs, much of the interest on this single carbon atom-thick material has nowadays also embraced other members of this large family such as few-layer graphene, GO, rGO, graphene ribbons, and graphene dots, to mention a few. GBMs - which present a high percentage of $\mathrm{sp}^{2}$ carbon atoms and a large aspect-ratio as common characteristics - show interesting features such as a rich redox chemistry and good electron/hole mobility.

These properties vary, however, significantly from one GBM to another as a consequence of their highly heterogeneous composition - different ratio of $\mathrm{sp}^{2}$ carbon and other-thancarbon atoms - and structure - different shapes and/or number of layers. In this context, STM and Raman spectroscopy stands out as two of the most powerful techniques to characterize GBMs, helping in distinguishing, for example, the morphology and electronic structure of GBMs (i.e., STM) or the number of layers, defects or crystal disorder, edge structures, changes in the hybridization, and doping effects (i.e., Raman spectroscopy).

Although, initially, pristine materials have been the preferred playground for researchers dealing with GBMs, especially in physics or materials science, the chemical functionalized of these GMBs has soon appeared as an interesting opportunity to prepare GBM-based hybrid materials showing tuneable physicochemical properties.

To meet such an ambitious objective, two main functionalization strategies, that is, the covalent and the non-covalent one, have been actively pursued. The former relies on the formation of one or more covalent bonds between GBMs - mostly, single or few-layer graphene - and the organic addend(s). In this context, cycloaddition and insertion reactions, 
synthetic protocols largely employed for the functionalization of fullerenes and carbon nanotubes, have been adapted to the GBMs' chemistry. However, the reduced presence of strained carbon atoms renders GBMs significantly less reactive than fullerenes and carbon nanotubes furnishing, in general, materials with a low degree of functionalization.

An elegant solution to this limitation has recently been proposed for graphitic materials and is extensively reviewed in this contribution. It relies on the formation of graphenides, that is, negatively-charged graphene sheets, as a product of the chemical reduction of graphite with alkaline metals. The formation of covalently-linked GBMs is finally achieved by such reactive graphenides attacking suitable electrophiles. Alternatively, an electron transfer from the graphenides to appropriate reagents can take place forming radical species, which subsequently undergo addition reactions to the graphene sheets. This strategy allows to generate covalent adducts exhibiting high degree of functionalization. As a drawback of this functionalization route, one could identify the incompatibility between the use of more "delicate" electroactive addends and the generation of highly reduced graphitic species due to the extreme reactivity of the latter. This problem could be circumvented by using the reductive functionalization to first install onto the graphene material small molecules bearing functional groups (e.g.: ethynyl, azide, alcohols, etc.) that could be reacted, in a following step, to appropriate electroactive species.

Turning to the non-covalent functionalization, it offers, undeniably, significant advantages with respect to the covalent approach, mainly, the preservation of the GBMs' electronic and band gap features, an aspect of primary importance for optoelectronic applications. Among the synthetic protocols, methods such as the sonication-induced liquid-phase exfoliation, or the use of surfactants, polymers or small aromatic molecules are most frequently used. As a result, stable graphene suspensions have been realized using derivatives bearing pyrenes, tetrathiafulvalenes, or perylene bisimides, the latter moieties able to strongly adhere to the graphene surface mainly through $\pi$-stacking interactions. Recently, non-covalent forces have also been employed for the functionalization of surface-supported graphene - a material, which is of high technological relevance for devices.

One of the most society-relevant applications of functionalized GBM hybrid materials is in the field of solar energy conversion. In these systems, Pcs and Pors, two families of macrocyclic compounds with remarkable structural, optical, and redox features, are among 
the most frequently used partners for single and few-layer graphene, GO, rGO, etc. To date, several examples of the covalent and non-covalent functionalized GMBs have been reported in which the presence of Pcs or Pors triggers the formation of long-lived, photoinduced charge separation. Quite intriguing is the fact that, in these systems, the direction of electron transfer, namely, from the GBM to the macrocycle, or vice versa, is controllable by the careful selection of the GBM and the redox feature of the porphyrinoid. Despite the fact that both Pcs and Pors feature a large number of requisites that an ideal electro- and photoactive material should have, the photovoltaic performances achieved with most of these macrocycle/GBM hybrids are still far from what is needed for real applications. Further room for improvements could be found by tackling aspects such as a better structural, optical, and electronic complementarity between the GBM and the Pc/Por, or a better dispersability and homogeneity of the resulting GBM-based hybrid materials. Incontestably, significant advances have been made during the last decade in the preparation, characterization and study of GBM-based hybrid materials. ${ }^{189}$ Nevertheless, a deeper understanding is still needed to precisely control the structural/compositional features, to establish reliable structure-property relationships, and to fine tune the physicochemical properties of these materials. In this context, several challenges and yet unsolved problems remain. For example, a somehow important issue, which should by no means be underestimated when dealing with the preparation of covalent or non-covalent GBM-based hybrid materials, is the presence of several chemically reactive and binding sites distributed across the GBMs' surface. As a result, the degree of functionalization of these functionalized materials cannot be satisfyingly controlled. This problem, together with the difficulties to control the size/composition of the GBMs and to reproduce them from batch to batch calls for a critical analysis of the experimental data. On a similar note, the exact structural characterization of GBM-based hybrid materials still represents a major problem, strongly relying on microscopic techniques such as STM and HR-TEM.

Properly addressing and, eventually, solving these problems and limitations, is expected to greatly boost, in the near future, the implementation of GBM-based hybrid materials in technologically-relevant applications. Moreover, the efforts put into developing novel synthetic strategies for the preparation of GBMs and novel techniques for their characterization could also result extremely valuable for exploring the chemistry of other 2- 
D materials or yet unknown synthetic carbon allotropes. We hope that this review, which provides an outlook on some fundamental aspects of GBMs such as their characterization, chemical functionalization, and applications in solar energy conversion and catalytic schemes, will foster the interest of scientists from different research areas to tackle the many challenges, to realize the high expectations, and to profit from the vast opportunities that GBM-based hybrid materials offer.

\section{List of abbreviations}

$\mathrm{AFM}=$ atomic force microscopy

$\mathrm{CCG}=$ chemically converted graphene

2-D $=2$-dimensional

3-D = 3-dimensional

$\mathrm{D}-\mathrm{A}=$ donor-acceptor

$o$-DCB $=o$-dichlorobenzene

GBMs $=$ graphene-based materials

$\mathrm{GICs}=$ graphite intercalation compounds

$\mathrm{GO}=$ graphene oxide

ILs $=$ ionic liquids

IPCE $=$ incident photon-to-photocurrent-efficiency

$\mathrm{NIR}=$ near-infrared

PAHs $=$ polycyclic aromatic hydrocarbons

$\mathrm{Pc}=$ phthalocyanine

PET $=$ photoinduced electron transfer

Por $=$ porphyrin

$\mathrm{rGO}=$ reduced graphene oxide

$\mathrm{SEM}=$ scanning electron microscopy

STM = scanning tunneling microscopy

$\mathrm{TEM}=$ transmission electron microscopy

$\mathrm{TGA}=$ thermogravimetric analysis

SubPc $=$ subphthalocyanine 


\section{Acknowledgements}

We are grateful for the financial support of the MINECO, Spain (CTQ2014-52869-P, CTQ2014-52045-R and CTQ2015-71936-REDT), the Comunidad de Madrid, Spain (FOTOCARBON, S2013/MIT-2841), the European Research Council (ERC-320441Chiralcarbon), and the National Science Foundation, USA (grant no. 1401188).

\section{Authors' biographies}

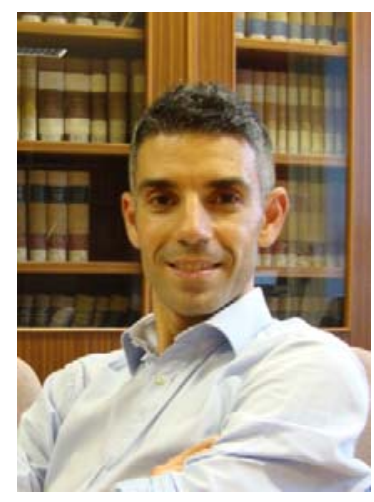

Giovanni Bottari received his B.S. degree from the University of Messina (Italy) and his $\mathrm{PhD}$ from the University of Edinburgh (United Kingdom). He was a Marie Curie IntraEuropean and a "Ramón y Cajal" Fellow at Universidad Autónoma de Madrid (UAM). Currently, he is Assistant Professor of Organic Chemistry at UAM, and Associate Scientist both at "IMDEA Nanoscience" and at the "Institute for Advanced Research in Chemical Sciences". His current research interests include the synthesis and study of covalent and supramolecular (sub)phthalocyanine-based systems and their self-organization both in solution and on surfaces.

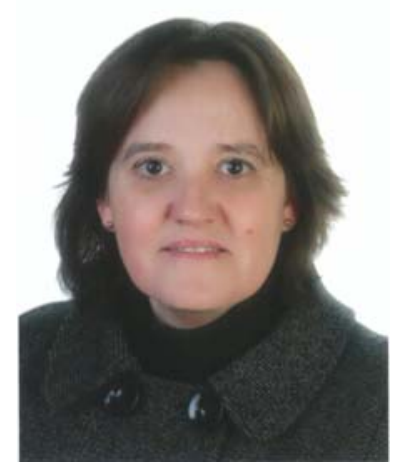


$\mathbf{M}^{\mathbf{a}}$ Ángeles Herranz obtained her PhD from Universidad Complutense de Madrid (UCM) and carried out postdoctoral stays at University of Miami (Florida, USA) and at Clemson University (South Carolina, USA), before joining in 2004 the group of Prof. Nazario Martín at UCM holding a Ramón y Cajal research associate position (2004-2007). Afterwards she became Assistant Professor (2007-2009) and from 2009 she is Associate Professor of Chemistry. Her current research interests, documented in about 100 scientific papers, reviews and book chapter contributions, focus on the chemistry, electronic and chiroptical properties of carbon nanostructures.

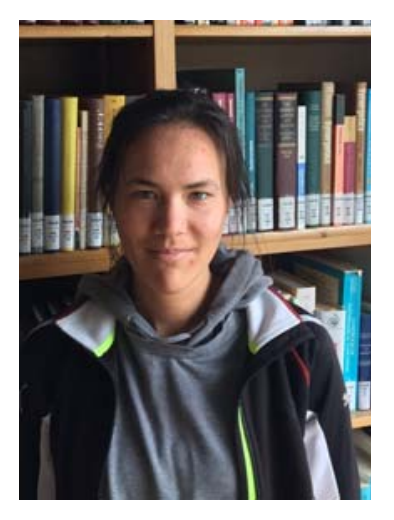

Leonie Wibmer obtained her master's degree in Molecular Nano Science in 2013 at the Friedrich-Alexander University of Erlangen-Nuremberg (Germany). She is currently doing her $\mathrm{PhD}$ in the group of Prof. Dirk M. Guldi. The focus of her work lies in the investigation of novel 2-D materials like graphene or $\mathrm{MoS}_{2}$, especially, in the context of establishing donor-acceptor hybrids with different chromophores.

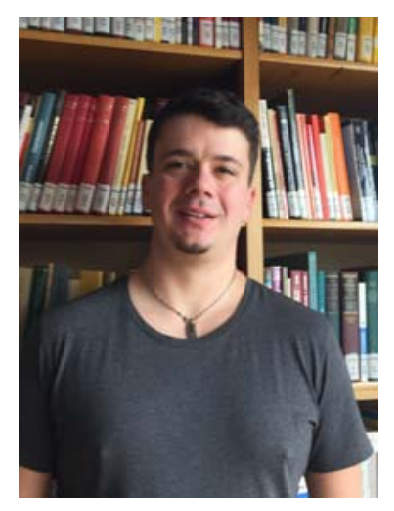


Michel Volland was born in Werneck (Germany) in 1988. He studied Molecular Nano Science and received his master's Degree in 2014 from the Friedrich-Alexander University of Erlangen-Nuremberg (Germany). Currently he is a $\mathrm{PhD}$ student in the research group of Prof. Dirk M. Guldi. His research covers charge transfer reactions in donor-acceptor graphene hybrid systems.

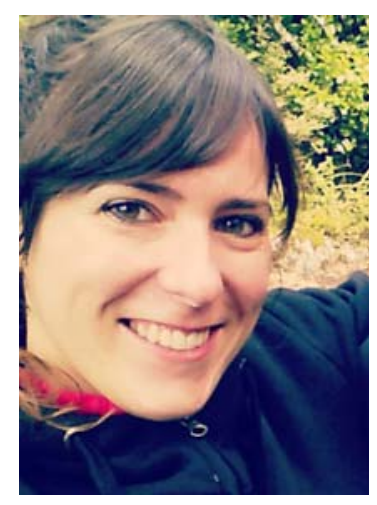

Laura Rodríguez-Pérez was born in Oviedo (Spain) in 1981. After spending a school year in the "Ecole Européene de Chimie Polymères et Matériaux-Université Louis Pasteur" of Strasbourg, she graduated in 2005 from the Universidad de Oviedo. In 2009 she received her $\mathrm{PhD}$ from the University of Toulouse. Then, she moved to Madrid to join the group of "Organic Molecular Materials" of Professor Nazario Martín León where she is currently engaged in the synthesis, functionalization and characterization of carbon nanoforms such us carbon nanotubes, graphene, fullerenes and graphene quantum dots.

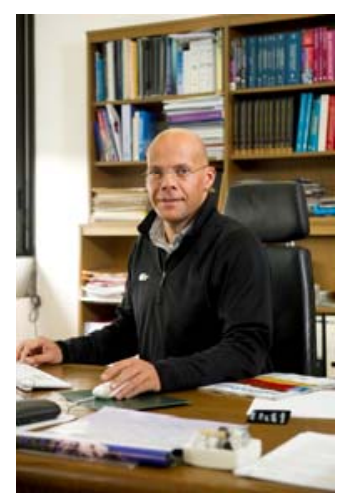

Dirk M. Guldi completed both his undergraduate studies (1988) and PhD (1990) at the University of Cologne (Germany). Following postdoctoral appointments at the National Institute of Standards and Technology (USA), the Hahn-Meitner Institute Berlin (1992), 
and Syracuse University, he joined the faculty of the Notre Dame Radiation Laboratory in 1995. Since 2004, he is Full Professor in the Department of Chemistry and Pharmacy at the Friedrich-Alexander University in Erlangen. Since 2013, Dirk M. Guldi is Associate Editor of Nanoscale, the RSC journal focused on experimental and theoretical research in all areas of nanotechnology and nanoscience, and he has been named among the world's 2014 and 2015 Highly Cited Researchers by Thomson Reuters.

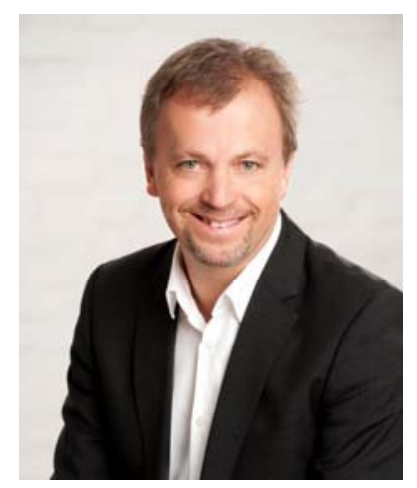

Andreas Hirsch obtained his $\mathrm{PhD}$ in organic chemistry in 1990. He has subsequently carried out postdoctoral research at the Institute for Polymers and Organic Solids in Santa Barbara, California. In 1991 he returned to Tübingen as a research associate. After his Habilitation in 1994 he joined the faculty of the Department of Chemistry at the University of Karlsruhe as a professor. Since October 1995, he has been Full Professor of Organic Chemistry at Friedrich-Alexander-Universität, Erlangen-Nürnberg. Andreas Hirsch's main research activities have been focused on the chemistry of synthetic carbon allotropes such as fullerenes, nanotubes, and graphene as well as supramolecular and nanochemistry.

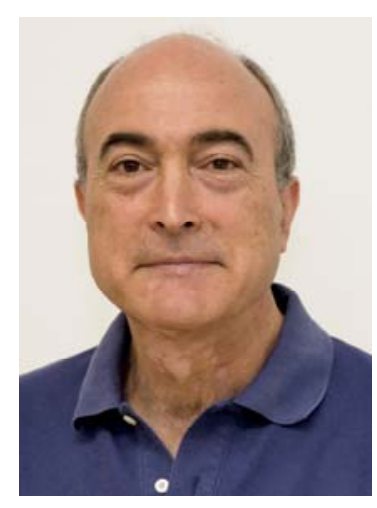


Nazario Martín, FRSC, is full professor at UCM and vice-director of IMDEANanoscience. Dr. h.c. by La Havana and Castilla La Mancha Universities, his research interests are focused on molecular and supramolecular chemistry of carbon nanostructures in the context of chirality, electron transfer, photovoltaic applications and nanoscience. He has published over 500 papers and supervised 34 theses. He is currently a member of the IAB of several international journals, including Chem. Soc. Rev., and he is the Editor-inChief of The Journal of Materials Chemistry (A, B and C). He is currently the President of the Confederation of Scientific Societies of Spain (COSCE). He has the "Advanced Grant" of the European Research Council (ERC) entitled "Chirallcarbon".

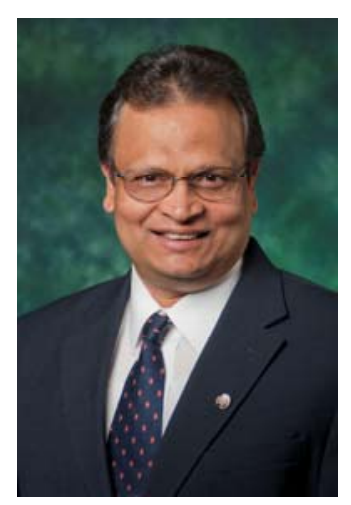

Francis D'Souza, FRSC, is a University Distinguished Research Professor of Chemistry and Materials Science and Engineering. He is part of UNT's Applied Materials and Manufacturing Processing Institute (AMMPI). Prior joining UNT in 2011, he was a Professor of Chemistry at Wichita State University, Wichita, KS. He received PhD (1992) from the Indian Institute of Science, Bangalore, India and post-doctoral studies at the University of Houston and University of Dijon, France. Dr. D'Souza's research covers wide areas of chemistry, nanophotonics and materials science. Principal research interests include chemistry and supramolecular chemistry of porphyrins and carbon nanomaterials, light energy harvesting, photoelectrochemistry and photovoltaics, electrochemical and photochemical sensors and catalysts, fluorescent chemosensors and biosensors, conducting nanocomposite hybrid materials for energy storage and conversion. Dr. D'Souza is an associate editor of Electrochemical Society (ECS) Journals and Journal of Porphyrins and Phthalocyanines, and is editorial board member of several other journals. 


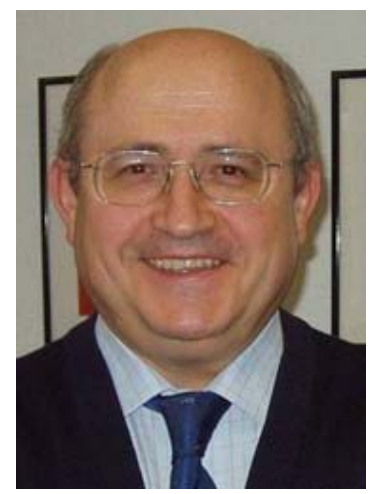

Tomás Torres is Full Professor of Organic Chemistry at the Autónoma University of Madrid (UAM) and Associated Senior Scientist at the IMDEA Nanoscience. In addition to various aspects of synthetic and supramolecular chemistry, his current research interests include the preparation and study of optical properties of organic functional materials. His group, that presently consists of twenty five researchers, is currently exploring several areas of basic research and applications of phthalocyanines, porphyrins and carbon nanostructures (fullerenes, carbon nanotubes, graphene), including organic and hybrid solar cells, with a focus on nanotechnology.

\section{References}

1 K. S. Novoselov, A. K. Geim, S. V. Morozov, D. Jiang, Y. Zhang, S. V. Dubonos, I. V. Grigorieva and A. A. Firsov, Science, 2004, 306, 666-669.

2 S. Eigler in Physics and Applications of Graphene-Experiments (Ed.: S. Mikhailov), InTech, New York, 2011, pp. 109.

3 P. Wick, A. E. Louw-Gaume, M. Kucki, H. F. Krug, K. Kostarelos, B. Fadeel, K. A. Dawson, A. Salvati, E. Vázquez, L. Ballerini, M. Tretiach, F. Benfenati, E. Flahaut, L. Gauthier, M. Prato and A. Bianco, Angew. Chem. Int. Ed., 2014, 53, 7714-7718.

4 S. R. Dhakate, N. Chauhan, S. Sharma, J. Tawale, S. Singh, P. D. Sahare and R. B. Mathur, Carbon, 2011, 49, 1946-1954.

5 A. C. Ferrari and D. M. Basko, Nat. Nano, 2013, 8, 235-246.

6 M. S. Dresselhaus, A. Jorio, M. Hofmann, G. Dresselhaus and R. Saito, Nano Lett., 2010, 10, 751-758.

7 G. Bottari, G. de la Torre and T. Torres, Acc. Chem. Res., 2015, 48, 900-910.

8 G. Bottari, G. de la Torre, D. M. Guldi and T. Torres, Chem. Rev., 2010, 110, 6768-6816 
9 C. E. Hamilton, J. R. Lomeda, Z. Sun, J. M. Tour and A. R. Barron, Nano Lett., 2009, 9 , 3460-3462.

10 Z. Jin, T. P. McNicholas, C.-J. Shih, Q. H. Wang, G. L. C. Paulus, A. J. Hilmer, S. Shimizu and M. S. Strano, Chem. Mater., 2011, 23, 3362-3370.

11 P. Huang, L. Jing, H. Zhu and X. Gao, Acc. Chem. Res., 2013, 46, 43-52.

12 Z. Xia, F. Leonardi, M. Gobbi, Y. Liu, V. Bellani, A. Liscio, A. Kovtun, R. Li, X. Feng, E. Orgiu, P. Samorí, E. Treossi and V. Palermo, ACS Nano, 2016, 10, 7125-7134.

13 A. Ejigu, I. A. Kinloch and R. A. W. Dryfe, ACS Appl. Mater. Interfaces, 2017, 9, 710-721.

14 M. Quintana, K. Spyrou, M. Grzelczak, W. R. Browne, P. Rudolf and M. Prato, ACS Nano, 2010, 4, 3527-3533.

15 L. H. Liu, M. M. Lerner and M. Yan, Nano Lett., 2010, 10, 3754-3756.

16 C. K. Chua, A. Ambrosi and M. Pumera, Chem. Commun., 2012, 48, 5376-5378.

17 S. Sarkar, E. Bekyarova, S. Niyogi and R. C. Haddon, J. Am. Chem. Soc., 2011, 133, 33243327.

18 C. K. Chua and M. Pumera, Chem. Soc. Rev., 2013, 42, 3222-3233.

19 S. Eigler and A. Hirsch, Angew. Chem. Int. Ed., 2014, 53, 7720-7738.

20 E. Vazquez, F. Giacalone and M. Prato, Chem. Soc. Rev., 2014, 43, 58-69.

21 A. Criado, M. Melchionna, S. Marchesan and M. Prato, Angew. Chem. Int. Ed., 2015, 54, 10734-10750.

22 V. Georgakilas, J. N. Tiwari, K. C. Kemp, J. A. Perman, A. B. Bourlinos, K. S. Kim and R. Zboril, Chem. Rev., 2016, 116, 5464-5519.

23 F. D’Souza, A. S. D. Sandanayaka and O. Ito. J. Phys. Chem. Lett., 2010, 1, 2586-2593.

24 F. D'Souza and O. Ito, Chem. Soc. Rev., 2012, 41, 86-96.

25 J. A. Mann and W. R. Dichtel, Phys. Chem. Lett., 2013, 4, 2649-2657.

26 A. Ciesielski and P. Samori, Adv. Mater., 2016, 28, 6030-6051.

27 M. Quintana, E. Vazquez and M. Prato, Acc. Chem. Res., 2013, 46, 138-148.

28 A. Ciesielski and P. Samori, Chem. Soc. Rev., 2014, 43, 381-398.

29 L. Rodríguez-Pérez, M. A. Herranz and N. Martín, Chem. Commun., 2013, 49, 3721-3735.

30 E. M. Pérez and N. Martín, Chem. Soc. Rev., 2015, 44, 6425-6433.

31 A. Luican-Mayer, G. Li and E. Y. Andrei, J. Electron Spectros. Relat. Phenomena, DOI: 10.1016/j.elspec.2017.01.005

32 Y. A. Eva, L. Guohong and D. Xu, Reports Prog. Phys., 2012, 75, 56501. 
33 J. Martin, N. Akerman, G. Ulbricht, T. Lohmann, J. H. Smet, K. von Klitzing and A. Yacoby, Nat. Phys., 2008, 4, 144-148.

34 E. Stolyarova, K. T. Rim, S. Ryu, J. Maultzsch, P. Kim, L. E. Brus, T. F. Heinz, M. S. Hybertsen and G. W. Flynn, Proc. Natl. Acad. Sci., 2007, 104, 9209-9212.

35 K. Xu, P. Cao and J. R. Heath, Nano Lett., 2009, 9, 4446-4451.

36 A. Deshpande, W. Bao, F. Miao, C. N. Lau and B. J. LeRoy, Phys. Rev. B, 2009, 79, 205411.

37 Y. Pan, H. Zhang, D. Shi, J. Sun, S. Du, F. Liu and H. Gao, Adv. Mater., 2009, 21, 2777-2780.

38 L. Gao, J. R. Guest and N. P. Guisinger, Nano Lett., 2010, 10, 3512-3516.

39 J. Xue, J. Sanchez-Yamagishi, D. Bulmash, P. Jacquod, A. Deshpande, K. Watanabe, T. Taniguchi, P. Jarillo-Herrero and B. J. LeRoy, Nat. Mater., 2011, 10, 282-285.

40 G. Li, A. Luican and E. Y. Andrei, Phys. Rev. Lett., 2009, 102, 176804.

41 R. Rozada, J. I. Paredes, M. J. López, S. Villar-Rodil, I. Cabria, J. A. Alonso, A. MartínezAlonso and J. M. D. Tascón, Nanoscale, 2015, 7, 2374-2390.

42 C. Gómez-Navarro, R. T. Weitz, A. M. Bittner, M. Scolari, A. Mews, M. Burghard and K. Kern, Nano Lett., 2007, 7, 3499-3503.

43 S. Obata, H. Tanaka and K. Saiki, Appl. Phys. Express, 2011, 4, 25102.

44 D. den Boer, J. G. Weis, C. A. Zuniga, S. A. Sydlik and T. M. Swager, Chem. Mater., 2014, 26, 4849-4855.

45 S. Kawai, S. Saito, S. Osumi, S. Yamaguchi, A. S. Foster, P. Spijker and E. Meyer, Nat. Commun., 2015, 6, 8098.

46 L. Zhao, R. He, K. T. Rim, T. Schiros, K. S. Kim, H. Zhou, C. Gutierrez, S. P. Chockalingam, C. J. Arguello, L. Palova, D. Nordlund, M. S. Hybertsen, D. R. Reichman, T. F. Heinz, P. Kim, A. Pinczuk, G. W. Flynn and A. N. Pasupathy, Science, 2011, 333, 999-1003.

47 R. Lv, Q. Li, A. R. Botello-Méndez, T. Hayashi, B. Wang, A. Berkdemir, Q. Hao, A. L. Elías, R. Cruz-Silva, H. R. Gutiérrez, Y. A. Kim, H. Muramatsu, J. Zhu, M. Endo, H. Terrones, J.-C. Charlier, M. Pan and M. Terrones, Sci. Rep., 2012, 2, 1-8.

48 L. M. Malard, M. A. Pimenta, G. Dresselhaus and M. S. Dresselhaus, Phys. Rep., 2009, 473, $51-87$.

49 M. Lazzeri, C. Attaccalite, L. Wirtz and F. Mauri, Phys. Rev. B, 2008, 78, 081406.

50 K. Yan, H. Peng, Y. Zhou, H. Li, Z. Liu, Nano Lett., 2011, 11, 1106-1110.

51 M. J. Matthews, M. A. Pimenta, G. Dresselhaus, M. S. Dresselhaus and M. Endo, Phys. Rev. $B$, 1999, 59, R6585-R6588. 
52 I. Pócsik, M. Hundhausen, M. Koós and L. Ley, J. Non. Cryst. Solids, 1998, 227-230, $1083-$ 1086.

53 A. Eckmann, A. Felten, I. Verzhbitskiy, R. Davey and C. Casiraghi, Phys. Rev. B, 2013, 88, 035426 .

54 L. G. Cançado, M. A Pimenta, B. R. A Neves, G. Medeiros-Ribeiro, T. Enoki, Y. Kobayashi, K. Takai, K.-I. Fukui, M. S. Dresselhaus, R. Saito and A. Jorio, Phys. Rev. Lett., 2004, 93, 047403.

55 L. G. Cançado, M. A. Pimenta, B. R. A. Neves, M. S. S. Dantas and A. Jorio, Phys. Rev. Lett., 2004, 93, 247401.

56 W. X. Wang, S. H. Liang, T. Yu, D. H. Li, Y. B. Li and X. F. Han, J. Appl. Phys., 2011, 109, $07 \mathrm{C} 501$.

57 X. Dong, D. Fu, W. Fang, Y. Shi, P. Chen and L.-J. Li, Small, 2009, 5, 1422-1426.

58 M. Kalbac, A. Reina-Cecco, H. Farhat, J. Kong, L. Kavan and M. S. Dresselhaus, ACS Nano, 2010, 4, 6055-6063.

59 O. Frank, M. S. Dresselhaus and M. Kalbac, Acc. Chem. Res., 2015, 48, 111-118.

60 A. Das, S. Pisana, B. Chakraborty, S. Piscanec, S. K. Saha, U. V Waghmare, K. S. Novoselov, H. R. Krishnamurthy, A. K. Geim, A. C. Ferrari and A. K. Sood, Nat. Nanotechnol., 2008, 3, 210-215.

61 A. Das, B. Chakraborty, S. Piscanec, S. Pisana, A. K. Sood and A. C. Ferrari, Phys. Rev. B, 2009, 79, 155417.

62 S. Niyogi, E. Bekyarova, M. E. Itkis, H. Zhang, K. Shepperd, J. Hicks, M. Sprinkle, C. Berger, C. N. Lau, W. A. deHeer, E. H. Conrad and R. C. Haddon, Nano Lett., 2010, 10, 4061-4066.

63 M. M. Lucchese, F. Stavale, E. H. M. Ferreira, C. Vilani, M. V. O. Moutinho, R. B. Capaz, C. A. Achete and A. Jorio, Carbon, 2010, 48, 1592-1597.

64 A. C. Ferrari and D. M. Basko, Nat. Nanotechnol., 2013, 8, 235-246.

65 L. G. Cançado, A. Jorio, E. H. M. Ferreira, F. Stavale, C. A. Achete, R. B. Capaz, M. V. O. Moutinho, A. Lombardo, T. S. Kulmala and A. C. Ferrari, Nano Lett., 2011, 11, 3190-3196.

66 S. Eigler, C. Dotzer and A. Hirsch, Carbon, 2012, 50, 3666-3673.

67 A. A. K. King, B. R. Davies, N. Noorbehesht, P. Newman, T. L. Church, A. T. Harris, J. M. Razal and A. I. Minett, Sci. Rep., 2016, 6, 19491.

68 R. J. Nemanich and S. A. Solin, Phys. Rev. B, 1979, 20, 392-401. 
69 G. P. Kotchey, B. L. Allen, H. Vedala, N. Yanamala, A. A. Kapralov, Y. Y. Tyurina, J. Klein-Seetharaman, V. E. Kagan and A. Star, ACS Nano, 2011, 5, 2098-2108.

70 D. Yang, A. Velamakanni, G. Bozoklu, S. Park, M. Stoller, R. D. Piner, S. Stankovich, I. Jung, D. A. Field, C. A. Ventrice and R. S. Ruoff, Carbon, 2009, 47, 145-152.

71 S. Stankovich, D. A. Dikin, R. D. Piner, K. A. Kohlhaas, A. Kleinhammes, Y. Jia, Y. Wu, S. T. Nguyen and R. S. Ruoff, Carbon, 2007, 45, 1558-1565.

72 D. C. Elias, R. R. Nair, T. M. G. Mohiuddin, S. V Morozov, P. Blake, M. P. Halsall, A. C. Ferrari, D. W. Boukhvalov, M. I. Katsnelson, A. K. Geim and K. S. Novoselov, Science, 2009, 323, 610-613.

73 M. Jaiswal, C. H. Y. X. Lim, Q. Bao, C. T. Toh, K. P. Loh and B. Ozyilmaz, ACS Nano, 2011, 5, 888-896.

74 R. A. Schäfer, J. M. Englert, P. Wehrfritz, W. Bauer, F. Hauke, T. Seyller and A. Hirsch, Angew. Chem. Int. Ed., 2013, 52, 754-757.

75 R. R. Nair, W. Ren, R. Jalil, I. Riaz, V. G. Kravets, L. Britnell, P. Blake, F. Schedin, A. S. Mayorov, S. Yuan, M. I. Katsnelson, H.-M. Cheng, W. Strupinski, L. G. Bulusheva, A. V Okotrub, I. V Grigorieva, A. N. Grigorenko, K. S. Novoselov and A. K. Geim, Small, 2010, 6, 2877-2884.

76 B. Wang, J. R. Sparks, H. R. Gutierrez, F. Okino, Q. Hao, Y. Tang, V. H. Crespi, J. O. Sofo and J. Zhu, Appl. Phys. Lett., 2010, 97, 141915.

77 A. Hirsch, J. M. Englert and F. Hauke, Acc. Chem. Res., 2013, 46, 87-96.

78 S. Eigler and A. Hirsch, Angew. Chem. Int. Ed., 2014, 126, 7852-7872.

79 M. J. Allen, V. C. Tung and R. B. Kaner, Chem. Rev., 2010, 110, 132-145.

80 V. Georgakilas, M. Otyepka, A. B. Bourlinos, V. Chandra, N. Kim, K. C. Kemp, P. Hobza, R. Zboril and K. S. Kim, Chem. Rev., 2012, 112, 6156-6214.

81 S. P. Economopoulos and N. Tagmatarchis, Chem. Eur. J., 2013, 19, 12930-12936.

82 Q. Tang, Z. Zhou and Z. Chen, Nanoscale, 2013, 5, 4541-4583.

83 J. M. Englert, C. Dotzer, G. Yang, M. Schmid, C. Papp, M. J. Gottfried, H. Steinrück, E. Spiecker, F. Hauke and A. Hirsch, Nat. Chem., 2011, 3, 279-286.

84 J. M. Englert, P. Vecera, K. C. Knirsch, R. A. Schäfer, F. Hauke and A. Hirsch, ACS Nano, 2013, 7, 5472-5482.

85 K. C. Knirsch, J. M. Englert, C. Dotzer, F. Hauke and A. Hirsch, Chem. Commun., 2013, 49, 10811-10813. 
86 F. Hof, R. A. Schäfer, C. Weiss, F. Hauke and A. Hirsch, Chem. Eur. J., 2014, 20, 16644-16651.

87 R. A. Schäfer, J. M. Englert, P. Wehrfritz, W. Bauer, F. Hauke, T. Seyller and A. Hirsch, Angew. Chem. Int. Ed., 2013, 52, 754-757.

88 Y. Vasilev, D. Wallis, M. Nuchter, B. Ondruschka, A. Lobach and T. Drewello, Chem. Commun., 2000, 1233-1234.

89 S. Pekker, J. P. Salvetat, E. Jakab, J. M. Bonard and L. Forro, J. Phys. Chem. B, 2001, 105, 7938-7943.

90 K. S. Subrahmanyam, P. Kumar, U. Maitra, A. Govindaraj, K. P. S. S. Hembram, U. V. Waghmare and C. N. R. Rao, Proc. Natl. Acad. Sci. USA, 2011, 108, 2674-2677.

91 V. Strauss, R. A. Schäfer, F. Hauke, A. Hirsch and D. M. Guldi, J. Am. Chem. Soc., 2015, 137, 40, 13079-13086.

92 R. A. Schäfer, D. Dasler, U. Mundloch, F. Hauke and A. Hirsch, J. Am. Chem. Soc., 2016, 138, 1647-1652.

93 K. C. Knirsch, R. A. Schäfer, F. Hauke and A. Hirsch, Angew. Chem. Int. Ed., 2016, 55, 5861-5864.

94 Z. Syrgiannis, B. Gebhardt, C. Dotzer, F. Hauke, R. Graupner and A. Hirsch, Angew. Chem. Int. Ed., 2010, 49, 3322-3325.

95 R. Kessinger, J. Crassous, A. Herrmann, M. Rüttimann, L. Echegoyen and F. Diederich, Angew. Chem. Int.Ed., 1998, 37, 1919-1922.

96 N. N. P. Moonen, C. Thilgen, L. Echegoyen and F. Diederich, Chem. Commun., 2000, $335-336$.

97 R. Schäfer, K. Weber, M. Kolesnik-Gray, F. Hauke, V. Krstic, B. Meyer and A. Hirsch, Angew. Chem. Int. Ed., 2016, 55, 14858-14862.

98 M. Biswal, X. Zhang, D. Schilter, T. K. Lee, D. Y. Hwang, M. Saxena, S. H. Lee, S. Chen, S. K. Kwak, C. W. Bielawski, W. S. Bacsa and R. S. Ruoff, J. Am. Chem. Soc., 2017, 139, $4202-4210$.

99 P. Vecera, J. Holzwarth, K. F. Edelthalhammer, U. Mundloch, H. Peterlik, F. Hauke and A. Hirsch, Nat. Commun., 2016, 7, 12411.

100 M. Yi and Z. Shen, J. Mater. Chem. A, 2015, 3, 11700-11715.

101 G. Cravotto and P. Cintas, Chem. Eur. J., 2010, 16, 5246-5259.

102 Y. Hernandez, V. Nicolosi, M. Lotya, F. M. Blighe, Z. Sun, S. De, I. T. McGovern, B. Holland, M. Byrne, Y. K. Gun'Ko, J. J. Boland, P. Niraj, G. Duesberg, S. Krishnamurthy, R. 
Goodhue, J. Hutchison, V. Scardaci, A. C. Ferrari and J. N. Coleman, Nat. Nanotechnol., 2008, 3, 563-568.

103 S. b. Haar, M. Bruna, J. X. Lian, F. Tomarchio, Y. Olivier, R. Mazzaro, V. Morandi, J. Moran, A. C. Ferrari, D. Beljonne, A. Ciesielski and P. Samorí, J. Phys. Chem. Lett., 2016, 7, 2714-2721.

104 U. Khan, A. O’Neill, M. Lotya, S. De and J. N. Coleman, Small, 2010, 6, 864-871.

105 K. R. Paton, E. Varrla, C. Backes, R. J. Smith, U. Khan, A. O’Neill, C. Boland, M. Lotya, O. M. Istrate, P. King, T. Higgins, S. Barwich, P. May, P. Puczkarski, I. Ahmed, M. Moebius, H. Pettersson, E. Long, J. Coelho, S. E. O’Brien, E. K. McGuire, B. M. Sanchez, G. S. Duesberg, N. McEvoy, T. J. Pennycook, C. Downing, A. Crossley, V. Nicolosi and J. N. Coleman, Nat. Mater., 2014, 13, 624-630.

106 C. E. Hamilton, J. R. Lomeda, Z. Sun, J. M. Tour and A. R. Barron, Nano Lett., 2009, 9, 3460-3462.

107 W. Du, X. Jiang and L. Zhu, J. Mater. Chem. A, 2013, 1, 10592-10606.

108 V. León, A. M. Rodriguez, P. Prieto, M. Prato and E. Vázquez, ACS Nano, 2014, 8, 563-571.

109 J. Shen, J. Wu, M. Wang, P. Dong, J. Xu, X. Li, X. Zhang, J. Yuan, X. Wang, M. Ye, R. Vajtai, J. Lou and P. M. Ajayan, Small, 2016, 12, 2741-2749.

110 U. Halim, C. R. Zheng, Y. Chen, Z. Lin, S. Jiang, R. Cheng, Y. Huang and X. Duan, Nat. Commun., 2013, 4, 2213.

111 M. Yi, Z. Shen, X. Zhang and S. Ma, J. Phys. D: Appl. Phys., 2013, 46, 025301.

112 A. B. Bourlinos, V. Georgakilas, R. Zboril, T. A. Steriotis and A. K. Stubos, Small, 2009, 5, $1841-1845$.

113 A. J. Oyer, J.-M. Y. Carrillo, C. C. Hire, H. C. Schniepp, A. D. Asandei, A. V. Dobrynin and D. H. Adamson, J. Am. Chem. Soc., 2012, 134, 5018-5021.

114 Z. Sun, X. Huang, F. Liu, X. Yang, C. Rosler, R. A. Fischer, M. Muhler and W. Schuhmann, Chem. Commun., 2014, 50, 10382-10385.

115 X. Wang, P. F. Fulvio, G. A. Baker, G. M. Veith, R. R. Unocic, S. M. Mahurin, M. Chi and S. Dai, Chem. Commun., 2010, 46, 4487-4489.

116 D. Nuvoli, L. Valentini, V. Alzari, S. Scognamillo, S. B. Bon, M. Piccinini, J. Illescas and A. Mariani, J. Mater. Chem., 2011, 21, 3428-3431.

117 R. Bari, G. Tamas, F. Irin, A. J. A. Aquino, M. J. Green and E. L. Quitevis, Colloids Surf. A, 2014, 463, 63-69.

118 M. Matsumoto, Y. Saito, C. Park, T. Fukushima and T. Aida, Nat. Chem., 2015, 7, 730-736. 
119 M. Lotya, Y. Hernandez, P. J. King, R. J. Smith, V. Nicolosi, L. S. Karlsson, F. M. Blighe, S. De, Z. Wang, I. T. McGovern, G. S. Duesberg and J. N. Coleman, J. Am. Chem. Soc., 2009, 131, 3611-3620.

120 M. Lotya, P. J. King, U. Khan, S. De and J. N. Coleman, ACS Nano, 2010, 4, 3155-3162.

121 S. Vadukumpully, J. Paul and S. Valiyaveettil, Carbon, 2009, 47, 3288-3294.

122 I. U. Unalan, C. Y. Wan, S. Trabattoni, L. Piergiovanni and S. Farris, RSC Adv., 2015, 5, 26482-26490.

123 H. W. Shim, K.-J. Ahn, K. Im, S. Noh, M.-S. Kim, Y. Lee, H. Choi and H. Yoon, Macromolecules, 2015, 48, 6628-6637.

124 T.-J. Ha, J. Lee, S. F. Chowdhury, D. Akinwande, P. J. Rossky and A. Dodabalapur, ACS Appl. Mater. Interfaces, 2013, 5, 16-20.

125 C. B. KC, G. N. Lim and F. D’Souza, Angew. Chem. Int. Ed., 2015, 54, 5088-5092.

126 F. G. Brunetti, H. Isla, J. Aragó, E. Ortí, E. M. Pérez and N. Martín, Chem. Eur. J., 2013, 19, 9843-9848.

127 J. M. Englert, J. Röhrl, C. D. Schmidt, R. Graupner, M. Hundhausen, F. Hauke and A. Hirsch, Adv. Mater., 2009, 21, 4265-4269.

128 L. Sánchez, R. Otero, J. M. Gallego, R. Miranda and N. Martín, Chem. Rev., 2009, 109, 2081-2091.

129 J. V. Barth, G. Constantini and K. Kern, Nature, 2005, 437, 671-679.

130 W. Lu and C. M. Lieber, Nat. Mater., 2007, 6, 841-850.

131 T.-C. Tseng, C. Urban, Y. Wang, R. Otero, S. L. Tait, M. Alcamí, D. Écija, M. Trelka, J. M. Gallego, N. Lin, M. Konuma, U. Starke, A. Nefedov, A. Langner, C. Wöll, M. A. Herranz, F. Martín, N. Martín, K. Kern and R. Miranda, Nat. Chem., 2010, 2, 374-379.

132 K. S. Mali, J. Greenwood, J. Adisoejoso, R. Phillipson and S. De Feyter, Nanoscale, 2015, 7, $1566-1585$.

133 W. Moritz, B. Wang, M. L. Bocquet, T. Brugger, T. Greber, J. Wintterlin and S. Günther, Phys. Rev. Lett., 2010, 104, 136102.

134 J. Wintterlin and M. L. Bocquet, Surf. Sci., 2009, 603, 1841-1852.

135 M. Roos, D. Künzel, B. Uhl, H.-H. Huang, O. B. Alves, H. E. Hoster, A. Gross and R. Jürgen Behm, J. Am. Chem. Soc., 2011, 133, 9208-9211.

136 X. Sun, J. Zhang, X. Wang, C. Zhang, P. Hu, Y. Mu, X. Wan, Z. Guo and S. Lei, Chem. Commun., 2013, 49, 10317-10319. 
137 S. Barja, M. Garnica, J. J. Hinarejos, A. L. Vázquez de Parga, N. Martín and R. Miranda, Chem. Commun., 2010, 46, 8198-8200.

138 M. Garnica, D. Stradi, S. Barja, F. Calleja, C. Díaz, M. Alcamí, N. Martín, A. L. Vázquez de Parga, F. Martín and R. Miranda, Nat. Phys., 2013, 9, 368-374.

139 C.-A. Di, D. Wei, G. Yu, Y. Liu, Y. Guo and D. Zhu, Adv. Mater., 2008, 20, 3289-3293.

140 J. S. Wu, W. Pisula and K. Müllen, Chem. Rev., 2007, 107, 718-747.

141 H. J. Jiang, Small, 2011, 7, 2413-2427.

142 I. V. Lightcap and P. V. Kamat, Acc. Chem. Res., 2013, 46, 2235-2243.

143 F. D'Souza and O. Ito, Sci. Progress, 2013, 96, 369-397.

144 A. Stergiou, G. Pagona and N. Tagmatarchis, Beilstein J. Nanotechnol., 2014, 5, 1580-1589.

145 Z. Li, M. He, D. Xu and Z. Liu, J. Photochem. Photobiol. C: Photochem. Reviews, 2014, 18, $1-17$.

146 G. Bottari, G. de la Torre and T. Torres, Acc. Chem. Res., 2015, 48, 900-910.

147 V. Strauss, A. Roth, M. Sekita and D. M. Guldi, Chem., 2016, 1, 531-556.

148 J. R. Lakowicz, Principles of Fluorescence Spectroscopy, $3^{\text {rd }}$ ed., Springer, Singapore, 2006.

149 Z. Y. Chen, S. Berciaud, C. Nuckolls, T.F. Heinz and L.E. Brus, ACS Nano, 2010, 4, 2964-2968.

150 R. S. Swathi and K. L. Sebastian, J. Chem. Phys., 2009, 130, 086101.

151 G. Williams and P. V. Kamat, Langmuir, 2009, 25, 13869-13873.

152 H. Chang, L. Tang, Y. Wang, J. Jiang and J. Li, Anal. Chem., 2010, 82, 2341-2346.

153 Z. Chen, S. Berciaud, C. Nuckolls, T. F. Heinz and L. E. Brus, ACS Nano, 2010, 4, 2964-2968.

154 K. P. Loh, Q. Bao, G. Eda and M. Chhowalla, Nat. Chem., 2010, 2, 1015-1024.

155 H. Dong, W. Gao, F. Yan, H. Ji and H. Ju, Anal. Chem., 2010, 82, 5511-5517.

156 H. S. S. R. Matte, K. S. Subrahmanyam, K. V. Rao, S. J. George and C. N. R. Rao, Chem. Phys. Lett., 2011, 506, 260-264.

157 C. B. KC., S. K. Das, K. Ohkubo, S. Fukuzumi and F. D’Souza, Chem. Commun., 2012, 48, 11859-11862.

158 J. Malig, N. Jux, D. Kiessling, J. J. Cid, P. Vázquez, T. Torres and D. M. Guldi, Angew. Chem. Int. Ed., 2011, 50, 3561-3565.

159 L. Brinkhaus, G. Katsukis, J. Malig, R. D. Costa, M. Garcia-Iglesias, P. Vázquez, T. Torres and D. M. Guldi, Small, 2013, 9, 2348-2357. 
160 D. Kiessling, R. D. Costa, G. Katsukis, J. Maling, F. Lodermeyer, S. Feihl, A. Roth, L. Wibmer, M. Kehrer, M. Volland, P. Wagner, G. G. Wallace, D. L. Officer and D. M. Guldi, Chem. Sci., 2013, 4, 3085-3098.

161 J. Malig, A. W. I. Stephenson, P. Wagner, G. G. Wallace, D. L. Officer and D. M. Guldi, Chem. Commun., 2012, 48, 8745-8747.

162 T. Skaltsas, S. Pispas and N. Tagmatarchis, Chem. Eur. J., 2013, 19, 9286-9290.

163 S. M. Aly, M. R. Parida, E. Alarousu and O. F. Mohammed, Chem. Commun., 2014, 50, 10452-10454.

164 A. Roth, M. E. Ragoussi, L. Wibmer, G. Katsukis, G. de la Torre, T. Torres and D. M. Guldi, Chem. Sci., 2014, 5, 3432-3438.

165 L. Wibmer, L. M. O. Lourenco, A. Roth, G. Katsukis, M. G. P. M. S. Neves, J. A. S. Cavaleiro, J. P. C. Tome, T. Torres and D. M. Guldi, Nanoscale, 2015, 7, 5674-5682.

166 S. P. Economopoulos and N. Tagmatarchis, J. Phys. Chem. C, 2015, 119, 8046-8053.

167 G. Jnawali, Y. Rao, J. H. Beck, N. Petrone, I. Kymissis, J. Hone and T. F. Heinz, ACS Nano, 2015, 9, 7175-7185.

168 A. K. Geim and K. S. Novoselov, Nat. Mater., 2007, 6, 183-191.

169 P. Avouris, M. Freitag and V. Perebeinos, Nat. Photon., 2008, 2, 341-350.

170 J. Misewich, R. Martel, P. Avouris, J. Tsang, S. Heinze and J. Tersoff, Science, 2003, 300, 783-786.

171 R. Lv, E. Cruz-Silva and M. Terrones, ACS Nano, 2014, 8, 4061-4069.

172 Y. Liu, F. Wang, Y. Liu, X. Wang, Y. Xu and R. Zhang, Nanoscale, 2016, 8, 12883-12886.

173 M. E. Ragoussi, J. Malig, G. Katsukis, B. Butz, E. Spiecker, G. de la Torre, T. Torres and D. M. Guldi, Angew. Chem. Int. Ed., 2012, 51, 6421-6425.

174 N. Karousis, J. Ortiz, K. Ohkubo, T. Hasobe, S. Fukuzumi, A. Sastre-Santos and N. Tagmatarchis, J. Phys. Chem. C, 2012, 116, 20564-20573.

175 M. E. Ragoussi, G. Katsukis, A. Roth, J. Malig, G. de la Torre, D. M. Guldi and T. Torres, J. Am. Chem. Soc., 2014, 136, 4593-4598.

176 Handbook of Carbon Nanomaterials, Eds. F. D’Souza and K. M. Kadish, World Scientific, Vol. 1-8, 2010-2016, Singapore.

177 Organic Nanomaterials, Eds. T. Torres and G. Bottari, Wiley, 2013, Hoboken, New Jersey

178 A. Wojcik and P. V. Kamat, ACS Nano, 2010, 4, 6697-6706.

179 M. Supur, K. Ohkubo and S. Fukuzumi, Chem. Commun., 2014, 50, 13359-13361.

180 M. Zhang, B. Yuan, S.-Z. Kang, L. Qin, G. Li and X. Li, RSC Adv., 2015, 5, 42063-42068. 
181 M. Supur, Y. Kawashima, K. Mase, K. Ohkubo, T. Hasobe and S. Fukuzumi, J. Phys. Chem. C, 2015, 119, 13488-13495.

182 N. Karousis, A. S. D. Sandanayaka, T. Hasobe, S. P. Economopoulous, E. Sarantopoulou and N. Tagmacharchis, J. Mater. Chem., 2011, 21, 109-117.

183 T. Umeyama, J. Mihara, N. Tezuka, Y. Matano, K. Stranius, V. Chukharev, N. V. Tkachenko, H. Lemmetyinen, K. Noda, K. Matsushige, T. Shishido, Z. Liu, K. Hirose-Takai, K. Suenaga and H. Imahori, Chem. Eur. J., 2012, 18, 4250-4257.

184 G. Kalita, S. Sharma, K. Wakita, M. Umeno, Y. Hayashi and M. Tanemura, Phys. Chem. Chem. Phys., 2013, 15, 1271-1274.

185 S. K. Das, C. B. KC, K. Ohkubo, Y. Yamada, S. Fukuzumi and F. D’Souza, Chem. Commun., 2013, 49, 2013-2015.

186 S. K. Das, C. B. KC and F. D'Souza, Fullerenes, Nanotubes, and Carbon Nanostructures, 2014, 22, 128-132.

187 H.-J. Kim, J. Sung, H. Chung, Y. J. Choi, D. Y. Kim and D. Kim, J. Phys. Chem. C, 2015, 119, $11327-11336$.

188 Z. Li, C. He, Z. Wang, Y. Gao, Y. Dong, C. Zhao, Z. Chen, Y. Wu and W. Song, Photochem. Photobiol. Sci., 2016, 15, 910-919.

189 A. C. Ferrari et al., Nanoscale, 2015, 7, 4598-4810. 